Bundesgesundheitsbl 2018 ·61:1310-1361 https://doi.org/10.1007/s00103-018-2811-2 (c) Springer-Verlag GmbH Deutschland, ein Teil von Springer Nature 2018

\section{Einleitung, Definitionen und Hintergrund}

\begin{abstract}
Allgemein gelten Enterokokken als nur bedingt pathogen und nicht sehr virulent, sie sind aber dennoch mit humanen Infektionen assoziiert. Zunehmend werden Enterokokken insbesondere in $\mathrm{Zu}-$ sammenhang mit speziellen Patientengruppen als Verursacher verschiedener nosokomialer Infektionen mit erheblicher Letalität (z. B. der Gefäßkatheter-assoziierten Sepsis) beschrieben und damit auch verstärkt im klinischen Alltag wahrgenommen [1].
\end{abstract}

Enterokokken sind in der Lage, extrachromosomale Elemente zu akquirieren, die Antibiotikaresistenzen kodieren. $\mathrm{Zu}$ den wichtigsten akquirierten Resistenzen gehört die Resistenz gegenüber Glykopeptidantibiotika. Bereits in den 90er Jahren wurden die Vancomycin-resistenten Enterokokken (VRE) in den USA als die multiresistenten Erreger der Dekade bezeichnet [2]. Zu dieser Zeit wurde auch in Deutschland eine Zunahme von VRE beobachtet, die jedoch mit dem Verbot von Avoparcin (ein Glykopeptid-Antibiotikum) als Wachstumsförderer in der Viehzucht wieder zurückging $[3,4]$. In jüngerer Zeit war eine erneute Zunahme der Nachweise von VRE zu beobachten [5] und Deutschland war eines der europäischen Länder mit der stärksten Zunahme [6]. Im Jahr 2017 wurde von der Weltgesundheitsorganisation (WHO) erstmals

\title{
Hygienemaßnahmen zur
} Prävention der Infektion durch Enterokokken mit speziellen Antibiotikaresistenzen

\author{
Empfehlung der Kommission \\ für Krankenhaushygiene und \\ Infektionsprävention (KRINKO) beim Robert \\ Koch-Institut
}

eine Liste von zwölf Bakteriengruppen veröffentlicht, die in der Forschung und Entwicklung neuer Antibiotika zukünftig Priorität haben sollten. VRE wurden in dieser Liste eine hohe Priorität zugewiesen [7].

Über die Zunahme der VRE-Nachweise hinaus finden sich in den letzten Jahren weitere Resistenzen auch gegen neue Reserveantibiotika wie Linezolid oder Tigecyclin [6].

Nachdem in Deutschland zunächst entsprechend den US-amerikanischen Empfehlungen zur Prävention der Verbreitung von VRE meist Screening von Patienten und Isolierung von VRE-Trägern eingesetzt wurden [8], findet derzeit eine kontroverse Diskussion zur Notwendigkeit der Isolierung solcher Patienten statt [9-11].

Anliegen der Kommission war es daher, auf Basis der vorliegenden Literatur eine Empfehlung zum Umgang mit $\mathrm{Pa}$ tienten mit Infektion oder Besiedelung mit VRE und darüber hinaus mit anderen antibiotikaresistenten Enterokokken zu erstellen. Die Maßnahmen sollen dabei die Epidemiologie, die Übertragungswege, die Pathogenität des Erregers und die Risikopopulationen einer Einrichtung genauso berücksichtigen wie die Verlässlichkeit der Prävention durch Basishygiene.

\subsection{Zielgruppe dieser Empfehlung}

Diese Empfehlung richtet sich primär an die Leiter und Mitarbeiter ${ }^{1}$ von Krankenhäusern und insbesondere an das Hygienefachpersonal. Auch in anderen medizinischen Einrichtungen, in denen mit Krankenhäusern vergleichbare Therapien, z. B. Beatmungen in der neurologischen Frührehabilitation, durchgeführt werden, kann sie hilfreich sein. Andere Einrichtungen, die den Lebensbereich der Patienten darstellen (Alten- und Pflegeheime), werden in dieser Empfehlung nicht berücksichtigt. Hier ist eine individuelle Risikoabwägung empfehlenswert, wie sie in den Empfehlungen zur Infektionsprävention in Heimen dargestellt wird [12].

\subsection{Bezug zu vorausgegangenen Empfehlungen der KRINKO}

Die Empfehlungen stellen Maßnahmen zur Prävention der Infektion mit Enterokokken mit speziellen Antibiotikaresistenzen zusammen. Hierbei wird ausdrücklich die endemische Situation oder das Auftreten von einzelnen Fällen behandelt. Für Maßnahmen, die bei Ausbrüchen zu ergreifen sind, sei auf die Empfehlungen zum Ausbruchsmanagement und strukturierten Vorgehen bei gehäuf-

\footnotetext{
1 Bei allen Berufs- oder Gruppenbezeichnungen sind grundsätzlich immer beide Geschlechter gemeint.
} 


\section{Inhalt}

1 Einleitung, Definitionen und Hintergrund

1.1 Zielgruppe dieser Empfehlung

1.2 Bezug zu vorausgegangenen Empfehlungen der KRINKO

1.3 Definitionen und Hintergrund

1.4 Mikrobiologische Diagnostik

1.5 Surveillance nach $\S 23$ IfSG

2 Epidemiologie der Infektion und Besiedelung mit antibiotikaresistenten Enterokokken

2.1 Eigenschaften und natürliches Habitat der Enterokokken (Reservoire, Persistenz in der Umgebung, Hitzeresistenz)

2.2 Epidemiologie klinisch bedeutsamer Resistenzmechanismen bei Enterokokken

2.2.1 Penicillin/Ampicillin

2.2.2 High-level Gentamicin

2.2.3 Glykopeptide

2.2.4 Oxazolidinone (Linezolid/Tedizolid)

2.2.5 Tigecyclin

2.2.6 Daptomycin

2.3 Bedeutung der Enterokokken als Infektionserreger

2.3.1 Pathogenität

2.3.2 Übertragung

2.3.3 Kolonisation

2.3.4 Dauer der Besiedelung

2.3.5 Nosokomiale Infektion

2.4 Outcome von Enterokokken-Infektionen in Abhängigkeit von der Antibiotikaresistenz

2.4.1 Outcome bei Infektionen mit high-level Gentamicin resistenten Enterokokken

2.4.2 Outcome bei Infektionen mit VRE

2.4.3 Outcome bei Infektionen mit Linezolid-resistenten Enterokokken

2.4.4 Outcome bei Infektionen mit Tigecyclin-resistenten Enterokokken

2.4.5 Outcome bei Infektionen mit Daptomycin-resistenten Enterokokken

2.5 Zusammenfassung der Bedeutung von Enterokokken mit speziellen Resistenzen

3 Präventionsmaßnahmen

3.1 Screening

3.1.1 Effektivität von Screening

3.1.2 Screeningmethoden

3.2 Isolierung

3.3 Maßnahmen der Basishygiene und Barrieremaßnahmen

3.4 Schulung des Personals

3.5 Einbeziehung von Patienten

3.6 Reinigungs- und Desinfektionsprogramme

3.7 Antiseptisches Waschen der Patienten

3.8 Maßnahmen der Eradikation

3.9 Antibiotic Stewardship

4 Internationale Empfehlungen zum Umgang mit VRE

5 Empfehlungen

5.1 Erkennen, Erfassung und Bewertung von Infektionen durch Enterokokken

5.2 Empfehlungen zur Prävention von Infektionen durch VRE

5.3 Mögliche Komponenten von Präventionsbündeln

5.3.1 Screening

5.3.2 Isolierung

5.3.3 Antiseptisches Waschen

5.3.4 Einbeziehung der Patienten in Hygienemaßnahmen

5.3.5 Intensivierte Reinigung und Desinfektion der Umgebung

5.4 Weitere Maßnahmen zur Prävention von VRE-Infektionen oder-Transmissionen

5.4.1 Antibiotic Stewardship-Programme

5.4.2 Maßnahmen zur Eradikation

5.5 Empfehlungen zur Prävention von Infektionen durch LRE oder LVRE

5.6 Empfehlungen zur Prävention von Infektionen durch Enterokokken mit anderen Antibiotikaresistenzen

5.7 Begleitende Maßnahmen im Rahmen der Umsetzung

6 Anlagen

7 Literatur tem Auftreten nosokomialer Infektionen verwiesen [13]. Diese Maßnahmen sind selbstverständlich auch erforderlich, wenn die Häufung Mikroorganismen mit anderen Resistenzmustern als den hier behandelten betrifft.

Grundlegende Maßnahmen zur Infektionsprävention sind den entsprechenden weiteren Empfehlungen der KRINKO $\mathrm{zu}$ entnehmen. Hier sei insbesondere auf die Empfehlungen zur Basishygiene [14], Händehygiene [15] und Flächendesinfektion [16] verwiesen. Spezielle Maßnahmen für besonders gefährdete Patientenpopulationen finden sich in den Empfehlungen „Anforderungen an die Hygiene bei der medizinischen Versorgung von immunsupprimierten Patienten " [17] und „Empfehlung zur Prävention nosokomialer Infektionen bei neonatologischen Intensivpflegepatienten mit einem Geburtsgewicht unter $1500 \mathrm{~g}$ " [18].

Die hier aufgeführten Empfehlungen sind mit Kategorien entsprechend der Mitteilung „Die Kategorien in der Richtlinie für Krankenhaushygiene und Infektionsprävention - Aktualisierung der Definitionen" von 2010 versehen (• Tab. 1) [19].

\subsection{Definitionen und Hintergrund}

Enterokokken besitzen eine natürliche Unempfindlichkeit gegenüber einer Vielzahl von Antibiotika (siehe Abschnitt 2.2).

Vor einiger Zeit wurde durch eine internationale Arbeitsgruppe ein Vorschlag für Standarddefinitionen multiresistenter (MDR), extensiv-resistenter (XDR) und panresistenter (PDR) Mikroorganismen vorgelegt [20]. Für die Klassifizierung der Enterokokken werden dabei die Antibiotika-Gruppen Aminoglykoside (Gentamicin -high-level, Streptomycin -high-level), Carbapeneme, Fluorchinolone, Glykopeptide, Glycylcycline, Lipopeptide, Oxazolidinone, Penicilline, Streptogramine und Tetracycline herangezogen. Dabei gelten Isolate mit Resistenz gegenüber mindestens drei Gruppen als MDR, Isolate die gegenüber zwei oder weniger der Gruppen empfindlich sind, als XDR und Isolate, die gegenüber allen Substanzgruppen unempfindlich sind, als PDR [20]. Bei dieser Definition werden alle AntibiotikaGruppen als gleichwertig betrachtet. Von 
Tab. 1 Kategorien in der Richtlinie für Krankenhaushygiene und Infektionsprävention (2010) [19]

\begin{tabular}{|c|c|c|c|c|c|}
\hline Kategorie IA & \multicolumn{5}{|c|}{$\begin{array}{l}\text { Diese Empfehlung basiert auf gut konzipierten systematischen Reviews oder einzelnen hochwertigen randomisierten } \\
\text { kontrollierten Studien. }\end{array}$} \\
\hline Kategorie IB & \multicolumn{5}{|c|}{$\begin{array}{l}\text { Diese Empfehlung basiert auf klinischen oder hochwertigen epidemiologischen Studien und strengen, plausiblen und } \\
\text { nachvollziehbaren theoretischen Ableitungen. }\end{array}$} \\
\hline Kategorie II & \multicolumn{5}{|c|}{$\begin{array}{l}\text { Diese Empfehlung basiert auf hinweisenden Studien/Untersuchungen und strengen, plausiblen und nachvollziehbaren } \\
\text { theoretischen Ableitungen. }\end{array}$} \\
\hline Kategorie III & \multicolumn{5}{|c|}{$\begin{array}{l}\text { Maßnahmen, über deren Wirksamkeit nur unzureichende oder widersprüchliche Hinweise vorliegen, deshalb ist eine } \\
\text { Empfehlung nicht möglich. }\end{array}$} \\
\hline Kategorie IV & \multicolumn{5}{|c|}{ Anforderungen, Maßnahmen und Verfahrensweisen, die durch allgemein geltende Rechtsvorschriften zu beachten sind. } \\
\hline \multicolumn{2}{|c|}{ Resistenz der Enterokokken (E) } & Vancomycin & Linezolid & High-level Gentamicin & Andere Antibiotika \\
\hline \multicolumn{2}{|l|}{ Vancomycin (V) } & VRE & LVRE & VRE & VRE \\
\hline \multicolumn{2}{|l|}{ Linezolid (L) } & LVRE & LRE & LRE & LRE \\
\hline \multicolumn{2}{|c|}{ High-level Gentamicin (HL-G) } & VRE & LRE & HL-GRE & HL-GRE \\
\hline \multicolumn{2}{|c|}{ Andere Antibiotika } & VRE & LRE & HL-GRE & $\begin{array}{l}\text { Keine spezifische } \mathrm{A} \\
\text { zung }\end{array}$ \\
\hline
\end{tabular}

den Autoren selbst wird betont, dass die Definition epidemiologischen Zwecken dient, aber nicht als Basis für die Infektionskontrolle geeignet ist.

Entsprechend hat sich die KRINKO entschlossen, die vorgeschlagene Definition nicht für die Erarbeitung von Präventionsstrategien zu Grunde zu legen.

Traditionell wird bei der Beschreibung der Epidemiologie von multiresistenten Enterokokken Vancomycin als Leitantibiotikum verwendet; die entsprechenden Isolate werden als Vancomycin-resistente Enterokokken (VRE) bezeichnet. Hierbei ist die Resistenz gegenüber dem Leitantibiotikum oft mit der Resistenz gegenüber weiteren Antibiotika vergesellschaftet, so dass die Abkürzung zum Synonym für multiresistente Enterokokken wurde.

Aufgrund der weiten Verbreitung und Akzeptanz des Begriffs VRE wird dieser Bezug zum Leitantibiotikum beibehalten.

Nach der Einführung neuerer Antibiotika (auch als Reserveantibiotika bezeichnet) haben sich auch hierzu entsprechende Resistenzeigenschaften entwickelt, die ebenfalls häufig mit Bezug zum Antibiotikum abgekürzt werden, z. B. Linezolidresistente Enterokokken (LRE).

Im Rahmen dieser Empfehlung werden folgende Abkürzungen verwendet (- Tab. 2):

- VRE: Vancomycin-resistente Enterokokken für Enterokokken-Isolate mit
Resistenz gegenüber Vancomycin unabhängig von der Enterokokken-Spezies und unabhängig davon, welche weiteren Resistenzen vorliegen, wobei bei zusätzlich bestehender LinezolidResistenz die Abkürzung LVRE verwendet wird.

- LRE: Linezolid-resistente Enterokokken für Enterokokken-Isolate mit Resistenz gegenüber Linezolid unabhängig von der Enterokokken-Spezies und unabhängig davon, welche weiteren Resistenzen vorliegen, wobei bei zusätzlich bestehender VancomycinResistenz die Abkürzung LVRE verwendet wird.

- VSE oder LSE: Vancomycin- bzw. Linezolid-sensible Enterokokken werden im klinisch-praktischen Bereich nicht mit Abkürzungen versehen. In dieser Empfehlung werden die Abkürzungen nur dann genutzt, wenn im Rahmen von Studien die entsprechenden Isolate den resistenten Isolaten gegenüber gestellt werden.

- LVRE: Linezolid-Vancomycin-resistenter Enterokokken für EnterokokkenIsolate mit Resistenz gegenüber Linezolid und Vancomycin unabhängig von der Enterokokken-Spezies.

- HL-GRE: High-level Gentamicinresistente Enterokokken für Enterokokken-Isolate mit hochgradiger Resistenz gegenüber Gentamicin un- abhängig von der Enterokokken-Spezies. Kombinierte Resistenzen werden hier nicht berücksichtigt. Liegt zusätzlich eine Resistenz gegenüber Vancomycin und/oder Linezolid vor, so werden nur die oben beschriebenen Abkürzungen gebraucht.

- Resistenzen gegenüber anderen Antibiotika werden nicht durch Abkürzungen charakterisiert.

\subsection{Mikrobiologische Diagnostik}

Mit den kulturbasierten, mikrobiologischen Standardmethoden werden Enterokokken in der Regel gut nachgewiesen. Für die Enterokokken-Spezies Enterococcus (E.) faecalis gelingt die Differenzierung auf Speziesebene mit kommerziellen biochemischen Testsystemen in der Regel gut, für E. faecium in etwas geringerem Ausmaß. Moderne Identifikationssysteme (MALDI TOF MS) sind in der Lage, nahezu $100 \%$ der E. faecalis- und E. faecium-Isolate korrekt zu identifizieren [21].

Auf die Differenzierung von Vancomycin-sensiblen Enterokokken auf Speziesebene wird bei Isolaten aus bestimmten Materialien (z.B. Enterokokken aus Urin) und/oder bei fraglicher klinischer Bedeutung (z.B. polymikrobielle Wundinfektion unter Beteiligung von Enterokokken) häufig verzichtet. Dies muss insbesondere bei der Erstel- 
lung und Bewertung von Resistenzstatistiken berücksichtigt werden.

Die verfügbaren Methoden zur Vorhersage antimikrobieller Empfindlichkeiten bei Enterokokken funktionieren generell zuverlässig. Die Korrelation zwischen phänotypisch-nachgewiesener Resistenz, vor allem bei Vancomycin, und einem genotypischen Nachweis von Resistenzgenen ist sehr gut. Schwierigkeiten bestehen in geringem Maße bei erworbener Vancomycin-Resistenz vom VanBTyp (siehe Abschnitt 2.2.3), da einzelne Stämme die Resistenz schwach ausprägen können (Minimale Hemmstoffkonzentration (MHK) für Vancomycin unterhalb des Grenzwerts möglich). In diesen Fällen sind die Vancomycin Etest ${ }^{\oplus}$ Makromethode oder ein genotypischer Nachweis des vanB-Gens mittels Nukleinsäureamplifikationstechnik (NAAT) verlässlicher.

Für die Typisierung von Enterokokken werden verschiedene Methoden eingesetzt. Als Goldstandard gilt derzeit noch die Analyse von Restriktionsfragmenten der chromosomalen DNA mit Hilfe der Pulsfeldgelelektrophorese (PFGE). Die Methode eignet sich sehr gut um verwandte Stämme zu identifizieren. Als schwieriger gestaltet sich eine Interpretation der Ergebnisse bei nur weitläufig verwandten Stämmen, da einzelne genetische Ereignisse zu substantiellen Änderungen in den DNA-Fragmentmustern führen und somit einen gewissen Zusammenhang verschleiern können [21]. Für populationsbezogene Analysen sollte bevorzugt auf DNA-sequenzbasierte Methoden wie die Multilocus-Sequenztypisierung (MLST) zurückgegriffen werden. Die MLST erlaubt die Zuweisung von Sequenztypen (z.B. ST117), die in phylogenetisch verwandte Gruppen zusammengefasst werden können (z. B. klonaler Komplex CC17). Vergleiche von VRE/ Enterokokken auf der Basis des Gesamtgenoms ermöglichen die ultimative Diskriminierungsfähigkeit und eignen sich sowohl für Ausbruchsanalysen als auch für die Erfassung von überregional verbreiteten Epidemiestämmen und könnten zukünftig als Goldstandard bewertet werden [22].

\subsection{Surveillance nach §23 IfSG}

Da es sich bei den hier behandelten Mikroorganismen um Erreger mit speziellen Resistenzen handelt, muss eine Surveillance entsprechend den Erläuterungen des Robert Koch-Institutes zur Surveillance von nosokomialen Infektionen sowie zur Erfassung von Erregern mit speziellen Resistenzen und Multiresistenzen gemäß $\$ 23$ Abs. 4 Infektionsschutzgesetz (IfSG) erfolgen [23]. Die hierbei zu erfassenden Daten dienen einer patientenbezogenen und nach Untersuchungszeitraum und Herkunft des Isolats (d.h. mindestens Abteilungsebene) aufgeschlüsselten Bewertung des Vorkommens von E. faecalis und E. faecium mit speziellen Resistenzen in der jeweiligen Einrichtung.

Aufgrund der epidemiologischen Relevanz der Erreger sollen die entsprechenden Daten monatlich in einer gesonderten Tabelle zusammengefasst werden, aus der die Zahl der betroffenen Patienten pro Organisationseinheit (z. B. Station) und Zeit unmittelbar hervorgeht. Die Erfassung und regelmäßige zeitnahe Bewertung ist geeignet, die Erkennung eines Clusters/Ausbruches (einer ungewöhnlichen Häufung) zu erleichtern und unterstützt die Umsetzung der vorliegenden Empfehlung.

\section{Epidemiologie der Infektion und Besiedelung mit antibiotikaresistenten Enterokokken}

\subsection{Eigenschaften und natürliches Habitat der Enterokokken (Reservoire, Persistenz in der Umgebung, Hitzeresistenz)}

Der Darm ist das bedeutendste Reservoir für Enterokokken. Innerhalb der Spezies E. faecalis und E. faecium existieren klonale Linien. Während einige Linien Mensch und Tier zumeist nur besiedeln (kommensale Enterokokken) existieren andere klonale Linien, die für den überwiegenden Teil der nosokomialen Infektionen verantwortlich sind (Hospital-assoziierte (HA) E. faecalis/ E. faecium). Letztere stellen auch den Großteil der antibiotika- und Vancomycin-resistenten Enterokokken. Auf- grund ihrer Umweltpersistenz müssen Reservoire von (antibiotikaresistenten) humanpathogenen Enterokokken in der unbelebten Umgebung der Patienten berücksichtigt werden.

Enterokokken bilden eine eigene Gattung innerhalb der Familie der Streptococcaceae. Es sind über 40 verschiedene Enterokokken-Spezies bekannt [24], wovon die meisten Spezies keine humanmedizinische Bedeutung besitzen. Medizinisch haben letztlich nur Isolate der Spezies E. faecalis und E. faecium größere Bedeutung erlangt; gelegentlich treten auch Isolate anderer Spezies als Infektionserreger auf (E. avium, E. hirae, E. durans, E. gallinarum, E. casseliflavus).

Vorkommen: Das natürliche Habitat von E. faecalis und E. faecium ist der Gastrointestinaltrakt. Enterokokken sind bei ca. $80 \%$ der Kinder und Erwachsenen kulturell nachweisbar [25]. Bei Neugeborenen wurde in einer Studie E. faecalis nachgewiesen, E. faecium jedoch nicht [26]. In dieser Studie wurde E. faecalis bei ca. $30 \%$ der Erwachsenen vorgefunden [26]. Enterococcus spp. besiedeln den Darm als Kommensalen, wobei sie üblicherweise nur einen kleinen Teil der kommensalen Mikrobiota ausmachen. Je Gramm Stuhl sind $10^{5}-10^{7} \mathrm{KBE}$ E. faecalis und $10^{4}-10^{5}$ KBE E. faecium anzüchtbar [27]. Neben dem distalen Gastrointestinaltrakt können Magen, Genitaltrakt, Oropharynx, Haut und Wunden mit Enterokokken kolonisiert sein.

Molekulare Epidemiologie und Pathogenitätsfaktoren: Aus Typisierungen von Stämmen mittels verschiedener molekularer Verfahren ist bekannt, dass nosokomiale Infektionen und Häufungen mit multi- und Vancomycin-resistenten Enterokokken durch bestimmte klonale Linien hervorgerufen werden.

Bei E. faecalis betrifft dies Isolate der mittels MLST bestimmten klonalen Komplexe CC2 und CC9. $\mathrm{Zu}$ diesen Linien zählen u. a. Stämme aus den USA, die eine Penicillinase bilden (in Europa sehr selten) und die häufig high-level Gentamicin-Resistenz besitzen (siehe Abschnitt 2.2.2). Isolate von $E$. faecalis unterschiedlicher phylogenetischer Linien zeigen allerdings keine Unterschiede in ihrer Pathogenität, z. B. in Tierexperimenten. Sie unterscheiden sich auch nicht signifikant hinsicht- 
lich des Besitzes von Virulenzfaktoren, die bei $E$. faecalis eher ubiquitär verbreitet sind [28-30]. So besitzen auch viele Besiedlungsisolate von Mensch und Tier die klassische Pathogenitätsinsel von E. faecalis. Dies ist für die Spezies E. faecium deutlich anders. Hier sind Isolate aus bestimmten klonalen Linien verantwortlich für die überwiegende Mehrzahl an nosokomialen Infektionen und Häufungen mit multiund Vancomycin-resistenten E. faecium. Diese Isolate lassen sich von klassischen Besiedlungsisolaten von Mensch und Tier abgrenzen. Sie unterscheiden sich hinsichtlich ihres Kerngenoms („core genome"), weswegen sie auch durch ältere Typisierungsverfahren wie AFLP, MLVA und letztlich MLST abzugrenzen waren (frühere Bezeichnungen sind klonaler Komplex C1 oder CC17). Zudem besitzen nur HA-E. faecium-Stämme als Teil des Zusatzgenoms („accessory genome“) bestimmte genomische Inseln und $\mathrm{z}$. T. eine Pathogenitätsinsel, welche u.a. das Biofilm-assoziierte esp-Gen kodieren kann [31]. Bestimmte genetische Marker sind ausschließlich bei Hospitalstämmen verbreitet und lassen sich für eine Differenzierung von kommensalen E. faecium heranziehen [32, 33]. Vancomycin-Resistenz ist in Deutschland und Europa fast nur mit der Spezies E. faecium assoziiert und tritt hier nahezu ausschließlich bei HA-Stammlinien auf. Die Zunahme invasiver nosokomialer Infektionen mit Enterokokken ist überproportional häufig mit HA-Stämmen von E. faecium assoziiert [34-38].

Persistenz: Enterokokken zeichnen sich durch eine erhebliche Widerstandsfähigkeit gegenüber Umwelteinflüssen aus. Sowohl E. faecalis als auch E. faecium können $60{ }^{\circ} \mathrm{C}$ für 30 min überleben [27, 39-42]. Die Persistenz von Enterokokken einschließlich VRE und VSE wurde auf trockenen Flächen mit fünf Tagen bis 30 Monate ermittelt [43-49]. Aufgrund ihrer hohen Resistenz und Persistenz gegen Umwelteinflüsse kommen Enterokokken in einem breiten Spektrum von Untersuchungsmaterialien außerhalb des Menschen vor (z. B. Staub, Lebensmittel, Wasser).

\subsection{Epidemiologie klinisch bedeutsamer Resistenzmechanismen bei Enterokokken}

Enterokokken besitzen eine natürliche Unempfindlichkeit gegen Cephalosporine, semisynthetische Penicilline (z.B. Oxacillin), Monobactame, Aminoglykoside (low-level Resistenz), Lincosamide und Polymyxine. Einzelne natürliche Resistenzen beschränken sich auf bestimmte Spezies wie eine Resistenz gegen Streptogramine und Mupirocin bei E. faecalis und low-level Vancomycin-Resistenz bei E. gallinarum und E. casseliflavus.

\subsubsection{Penicillin/Ampicillin}

Isolate von $E$. faecalis sind bis auf sehr seltene Ausnahmen Ampicillin-sensibel. HA-Stämme von E. faecium sind überwiegend Ampicillin-resistent; die Ursache sind Mutationen im Penicillin-bindenden Protein PBP5. Vancomycin-resistente $E$. faecium sind zu nahezu $100 \%$ auch resistent gegen Ampicillin.

Beta-Lactame wie Ampicillin (auch Amoxicillin und Piperacillin) sind wichtige Basis- und first-line-Antibiotika zur Behandlung von unkomplizierten Enterokokkeninfektionen sowie wichtige Kombinationspartner bei invasiven Infektionen mit E. faecalis. Die Resistenzsituation ist hierbei deutlich speziesabhängig. Eine Resistenz gegen Ampicillin ist bei E. faecalis sehr selten $(<1 \%)$, bei HA-Isolaten von E. faecium hingegen sehr weit verbreitet (>90\%). Kommensale Isolate von E. faecium wiederum zeigen eine deutlich niedrigere Ampicillin-Resistenz als HA-klonale Linien [34, 35].

In E. faecalis wird eine Resistenz gegen Ampicillin durch Penicillinasen (Beta-Lactamasen) kodiert, wobei die Penicillinasen in Staphylokokken und Enterokokken phylogenetisch ähnlich sind. E. faecalis-Stämme mit Penicillinasen wurden in den 1990er Jahren in den USA beschrieben und zeigten dort eine Tendenz zur überregionalen Verbreitung (epidemische Stämme; klonaler Komplex CC9) $[28,29,50]$. In anderen Teilen der Welt inklusive Europa und Deutschland sind diese Stämme sehr selten. Es ist davon auszugehen, dass Ampicillin-resistente Isolate von E. faecalis größtenteils fehldiagnostizierte Isolate von E. faecium darstellen. Mit der Umstellung von biochemischer Identifikation auf MALDI TOF MS-basierter Speziesdiagnostik ist der Anteil der „Ampicillin-resistenten“ $E$. faecalis-Isolate in den Surveillancedaten bereits kontinuierlich zurückgegangen, $\mathrm{da}$ letztere Methode wesentlich verlässlicher die korrekte Spezies diagnostiziert.

In E. faecium wird Resistenz gegen Ampicillin nicht durch Enzyme wie Penicillinasen (Beta-Lactamasen), sondern durch Mutationen im Gen des Penicillinbindenden Proteins 5 (PBP5 $\left.5_{\mathrm{Efm}}\right)$ kodiert [51-54]. Das $p b p 5_{E f m}$-Gen ist in einzelnen Stämmen mit anderen Resistenzgenen (wie z. B. vanB) assoziiert, somit genetisch verlinkt und mittels Konjugation auch zwischen verschiedenen Stämmen übertragbar [55]. Weitere Ursachen der Ampicillin-Resistenz in E. faecium sind möglich [51, 52]. Ampicillin-Resistenz in klinischen E. faecium-Isolaten ist eine sehr verbreitete Eigenschaft und tritt in $>90 \%$ der klinischen Isolate auf [56]. Klinisch relevante Isolate mit Vancomycin-Resistenz sind nahezu vollständig auch resistent gegen Ampicillin [57-59].

\subsubsection{High-level Gentamicin}

Gentamicin und Streptomycin wirken kombiniert mit einem Zellwand-wirksamen Antibiotikum (Glykopeptid, Penicillin) bei Enterokokken synergistisch, was lediglich bei der Behandlung von Endokarditis eine Bedeutung hat. Enterokokken besitzen eine low-level Resistenz gegen Aminoglykoside und können durch Erwerb bestimmter Resistenzgene eine high-level Resistenz erwerben, bei Letzterem erlischt die synergistische Wirkung.

Enterokokken sind natürlich resistent gegen Aminoglykoside. Einzelne Aminoglykoside (nur Gentamicin, Streptomycin) erzielen in Kombination mit einem Zellwand-wirksamen Antibiotikum (Glykopeptid, Penicillin) eine synergistische Wirkung. Insofern ist eine Erfassung der Empfindlichkeiten gegen Gentamicin (und ggf. Streptomycin) klinisch interessant. Die synergistische Wirkung wird speziell bei Endokarditiden gewünscht, weswegen die Diagnostik einer highlevel Gentamicin-Resistenz hierbei von Bedeutung ist. Daten aus Resistenz-Sur- 


\section{Tab. 3 Varianten der erworbenen Vancomycin-Resistenz in Enterococcus spp.}

\begin{tabular}{|c|c|c|c|c|c|c|c|c|}
\hline Resistenzphänotyp & VanA & $\operatorname{VanB}^{\mathbf{b}}$ & $\operatorname{VanD}^{\mathbf{b}}$ & VanE & VanG $^{\mathbf{b}}$ & VanL & VanM & $\operatorname{VanN}$ \\
\hline $\begin{array}{l}\text { Vancomycin MHK } \\
\text { (in } \mathrm{mg} / \mathrm{L} \text { ) }\end{array}$ & $16-1000$ & $\begin{array}{l}4-32 \\
(-1000)\end{array}$ & $16-512$ & $8-32$ & 16 & 8 & $128->256$ & $12-16$ \\
\hline $\begin{array}{l}\text { Teicoplanin MHK } \\
\text { (in } \mathrm{mg} / \mathrm{L} \text { ) }\end{array}$ & (4-) 16-512 & $0,5-1$ & $0,5-64^{c}$ & 0,5 & 0,5 & $\mathrm{E}$ & $0,75 / 96^{c}$ & 0,5 \\
\hline Expression & induzierbar & induzierbar & konstitutiv & induzierbar & induzierbar & induzierbar & induzierbar & konstitutiv \\
\hline Übertragbarkeit & $+/-$ & $+/-$ & - & - & + & - & + & $+/-$ \\
\hline $\begin{array}{l}\text { Vorkommen in } \\
\text { Enterococcus spp. }\end{array}$ & $\begin{array}{l}\text { E. faecium } \\
\text { E. faecalis } \\
\text { E. durans } \\
\text { E. hirae } \\
\text { E. gallinarum } \\
\text { E. casseliflavus } \\
\text { E. raffinosus } \\
\text { E. avium } \\
\text { E. mundtii } \\
\text { E. cecorum }\end{array}$ & $\begin{array}{l}\text { E. faecium } \\
\text { E. faecalis } \\
\text { E. durans } \\
\text { E. gallina- } \\
\text { rum }^{a}\end{array}$ & $\begin{array}{l}\text { E. faecium } \\
\text { E. faecalis } \\
\text { E. raffinosus } \\
\text { E. gallina- } \\
\text { rum }^{a}\end{array}$ & E. faecalis & E. faecalis & E. faecalis & E. faecium & E. faecium \\
\hline \multicolumn{9}{|c|}{$\begin{array}{l}\text { E empfindlich (keine MHK angegeben) } \\
\text { EErwerb von vanA-, vanB- oder vanD-Genen zusätzlich zu vanC1/vanC2 (selten), das vanB-Gen hat auch ein Reservoir in anaeroben Darmkommensalen (keine Entero- } \\
\text { kokken) } \\
\text { 'Subtypen existieren (vanB1-3, vanD1-5, vanG1-2) } \\
\text { 'verschiedene VanD- bzW. VanM-Stämme zeigten variable Teicoplanin MHK-Werte }\end{array}$} \\
\hline
\end{tabular}

veillance-Studien, z. B. EARS-Net, zeigen hier mittlere Prävalenzen (2016: ca. 30 \% im Durchschnitt) bei invasiven E. faecalisIsolaten europaweit, wobei die Schwankungsbreite von ca. $15 \%$ in Frankreich, Schweden und Norwegen bis $>50 \%$ in Rumänien reicht (Daten aus 2016 [60]). Für E. faecium-Isolate zeigen diese Daten ebenfalls mittlere bis hohe Prävalenzen von ca. $20 \%$ in Deutschland, Schweden und Griechenland und bis zu $80 \%$ in Rumänien und Bulgarien (Daten aus 2016 [60]). High-level Gentamicin-Resistenz wird in Staphylokokken und Enterokokken meist durch ein bifunktionales Enzym vermittelt. Das entsprechende Gen $a a c$ (6')-Ie- $a p h$ (2“)-Ia (syn. $a a c A-a p h D$ ) ist Teil eines mobilen Elements, Transposon Tn5281 bzw. Tn4001 [61].

\subsubsection{Glykopeptide}

Klinisch bedeutsame Vancomycin-Resistenz wird durch Gencluster vom Typ $v a n A$ und $v a n B$ kodiert und verbreitet sich in an das Krankenhausmilieu adaptierten E. faecium-Stämmen. Beide Varianten sind genetisch mobil, womit sich die Resistenz sowohl klonal als auch durch horizontalen Gentransfer (HGT) auf nicht verwandte Stämme verbreiten kann. Es existiert ein Reservoir der $v a n B$-vermittelten Vancomycin-Resis- tenz in Darmkommensalen (keine Enterokokken).

Acht verschiedene Varianten der erworbenen Vancomycin-Resistenz (VanAN) sind bisher beschrieben (• Tab. 3). Die Spezies E. gallinarum und E. casseliflavus (identisch mit E. flavescens [62]) besitzen eine natürliche Resistenz gegen Vancomycin (MHK von $2-32 \mu \mathrm{g} / \mathrm{mL}$ ), die als VanC-Phänotyp bezeichnet wird (VanC1 - E. gallinarum, VanC2 - E. casseliflavus). Erworbene Vancomycin-Resistenz betrifft nahezu ausschließlich Isolate der Spezies E. faecium, wohingegen Vancomycin-resistente $E$. faecalis und Isolate anderer Spezies selten sind. Von den acht derzeit bekannten Resistenztypen haben lediglich VanA und VanB medizinische Bedeutung erlangt, so dass im Folgenden auf diese beiden Varianten verstärkt eingegangen wird.

Resistenzen vom VanA-N-Typ sind durch eine Vielzahl genetischer Elemente kodiert und gehen mit einem relativ komplexen Resistenzmechanismus einher. Das Gencluster vom vanA- bzw. vanB-Typ ist mobil bzw. häufig auf übertragbaren Elementen lokalisiert und kann somit auch zwischen nicht verwandten Stämmen weitergegeben werden (• Tab. 3). Insofern ist sowohl die klonale Verbreitung als auch die horizontale Weitergabe der
Resistenz aus hygienischen und infektiologischen Gesichtspunkten zu bedenken. Eine Weitergabe eines Resistenzplasmids unter Ausbruchsbedingungen ist anscheinend bei VRE seltener als z. B. bei multiresistenten gramnegativen Bakterien (ESBLPlasmide) [63-65].

Die beiden häufigsten Resistenztypen vom VanA-und VanB-Typ sind in der Regel induzierbar, d.h. die Resistenzgene werden erst bei Anwesenheit des Antibiotikums exprimiert. Die VanB-vermittelte Resistenz wird nur durch Vancomycin, aber nicht durch Teicoplanin induziert. Daher sind VRE vom VanB-Typ in vitro Teicoplanin-empfindlich. Unter Teicoplanin-Therapie kann es zu einer Selektion gegen Teicoplanin konstitutiv-resistenter Mutanten kommen (Resistenzgene werden unabhängig von der Anwesenheit von Antibiotika kontinuierlich exprimiert), was den Erfolg einer TeicoplaninTherapie auch bei in vitro empfindlichen Enterokokken vom VanB-Typ mindern kann [66].

Die vanA- und vanB-tragenden E. faecium-Stämme unterscheiden sich in ihren übrigen Eigenschaften nicht grundsätzlich voneinander. Die beiden Resistenzgene bzw. -gencluster sind vor allem in HAStämmen nachweisbar (z.B. ST117, ST17, ST18; ehemals klonaler Komplex CC17) 
[58, 59, 63]. Diese HA-E. faecium-Stämme mit Ampicillin- und Fluorchinolon-Resistenz lassen sich als Glykopeptid-empfindliche Stämme bereits bei vielen Krankenhauspatienten nachweisen [6]. Sie sind zur klonalen Verbreitung innerhalb und zwischen Krankenhäusern fähig. Diese Stämme sind meist mit Virulenzmarkern (Esp und/oder Hyl als Marker für enterococcal surface protein bzw. putative Hyaluronidase) ausgestattet [32, 67-71]. HA-E. faecium-Stämme werden klinisch meist erst nach dem Erwerb von Glykopeptid-Resistenz-Determinanten (vanA- bzw. vanBGencluster) erkannt. Viele Stammvarianten können sowohl als VanA-Typ als auch als VanB-Typ VRE auftreten. Nichtsdestotrotz gibt es bestimmte erfolgreiche Stammvarianten, die eher als VanA-Typ (ST203) bzw. als VanB-Typ VRE (ST192) präsent sind [72, 73].

Das vanB-Gen kommt auch außerhalb der Enterokokken in Darmkommensalen vor $[74,75]$ was wiederum zu diagnostischen Unsicherheiten bei der Verwendung von NAAT führen kann und immer eine anschließende mikrobiologische Bestätigung erfordert (siehe Abschnitt 3.1) $[32,76]$. Beim vanB-Gen gibt es drei Subtypen, die mittels Sequenzierung identifiziert und differenziert werden können. Alle Subtypen werden von diagnostischen Assays erkannt. In den meisten klinischen Stämmen tritt die vanB2-Variante auf.

Epidemiologie: Der VanA-Typ ist weltweit der häufigste Glykopeptid-Resistenztyp. Für Deutschland deuten verschiedene Surveillance- und Referenzsysteme in den letzten Jahren eine ungefähre Gleichverteilung der VanA-Typ- und VanB-Typ-Resistenz in VRE an [77-81]. Ebenso setzten sich die VRE-Einsendungen an das Nationale Referenzzentrum für Enterokokken in den letzten Jahren zu nahezu gleichen Anteilen aus VanA- und VanB-VRE zusammen [57]. Aufgrund der relativ guten Übereinstimmung zwischen Genotyp und Phänotyp kann eine Ableitung des Genotyps aus dem Antibiogramm mit akzeptabler Sicherheit erfolgen (Vancomycinund Teicoplanin-resistente Isolate: VanA, Vancomycin-resistente und Teicoplaninsensible Isolate: VanB).

Die oben erwähnte überregionale Gleichverteilung kann im regionalen und lokalen Kontext deutlich anders sein und auch zeitlich variieren $[53,72,73]$, d.h. bestimmte Krankenhäuser oder Regionen sehen nur einen bestimmten (vorherrschenden) VRE-Typ, wobei dieser durch einen anderen abgelöst werden oder auch gleichzeitig mit einem anderen auftreten kann [53].

In Deutschland und benachbarten EUStaaten traten in den zurückliegenden Jahren (2012-2016) verstärkt VanB-VRE auf $[57,58,63,72]$. Dies betrifft sowohl Isolate aus endemischen Besiedlungen und Infektionen als auch Isolate aus Häufungen von Besiedlungen und Infektionen (Ausbrüche). Es ist denkbar, dass das häufigere Auftreten von VanB-Stämmen mit einer verbesserten Diagnostik von VanBVRE einhergeht. Trotzdem können bei einzelnen VanB-Stämmen die MHK-Werte auch unterhalb des seit einigen Jahren nach unten angepassten Grenzwertes für Vancomycin liegen (EUCAST) und diese niedrige Expression der VanB-Typ-Resistenz kann diagnostische Schwierigkeiten bei der Erkennung dieser Stämme verursachen [82].

Obwohl immer wieder diskutiert, gibt es derzeit keine belastbaren Daten und belegbaren Studien, die ein Reservoir der vanB vermittelten Vancomycin-Resistenz bei Enterokokken im ambulanten Bereich bzw. in der Allgemeinbevölkerung belegen. Für eine Krankenhaus-assoziierte Verbreitung spricht auch eine Prävalenzuntersuchung aus Irland. Dort waren $31,4 \%$ der von Krankenhauspatienten, aber $0 \%$ der von ambulanten Patienten entnommenen Stuhlproben VRE-positiv [83]. Da die Untersuchung anonymisiert erfolgte, ist nichts über vorbestehende Besiedelung bekannt.

Gentransfer auf S. aureus: Von besonderer Bedeutung ist das Risiko der Übertragung der Vancomycin-Resistenz auf andere Spezies, insbesondere auf $S$. aureus. Horizontaler Gentransfer der vanA-Gene zwischen Enterokokken und $S$. aureus wurde in vitro und in vivo beobachtet [84-86] und vor allem aus den USA [87] aber auch aus Europa (Portugal) beschrieben [88].

Auch wenn ein solcher Gentransfer in niedriger Frequenz zu erfolgen scheint, wurde empfohlen, im Rahmen von Infektionspräventionsprogrammen die Frequenz der Koinfektion durch VRE und
MRSA zu reduzieren, um der Möglichkeit des Gentransfers und damit der Infektion mit Vancomycin-resistenten $S$. aureus vorzubeugen [87].

\subsubsection{Oxazolidinone (Linezolid/ Tedizolid)}

Linezolid ist eine wichtige Therapieoption zur Behandlung von VRE-Infektionen. Resistenzen resultieren aus Punktmutationen in ribosomalen Genen. Es sind aber auch übertragbare Resistenzgene beschrieben worden ( $c f r, o p t r A)$. Das Auftreten von Linezolid-resistenten Isolaten korreliert meist mit einem erhöhten Einsatz der Substanz in betroffenen Einrichtungen, kann aber auch unabhängig davon auftreten.

Linezolid war das erste Antibiotikum der Gruppe der synthetischen Oxazolidinone [89]. Es verhindert die Initiation der Translation und hemmt die bakterielle Proteinsynthese. Linezolid wirkt bakteriostatisch. Es gilt als ein Reserveantibiotikum bei VRE-Infektionen. Tedizolid ist ein neueres Oxazolidinon mit verbesserter Wirksamkeit gegen grampositive Bakterien insgesamt [90]. Eine verbesserte Wirksamkeit gegen Enterokokken/VRE ist nicht sicher. Bei EUCAST existiert für Tedizolid zum Zeitpunkt der Veröffentlichung kein Grenzwert (IE=insufficient evidence), bei CLSI existiert für E. faecalis ein Grenzwert für die Sensibel-Kategorie (sensibel $\leq 0,5 \mathrm{mg} / \mathrm{L}$ ).

Enterokokken mit Resistenz gegen Linezolid sind bekannt. Die Linezolid-Resistenz wird dabei durch Mutationen in dem Gen, das die ribosomale 23S RNA kodiert, Mutationen ribosomaler Proteine (RplC, RplD) und/oder durch die Plasmid-vermittelte Resistenz vom $c f r$-Typ verursacht [91]. Das erstmals in Koagulase-negativen Staphylokokken vom Tier beschriebene $c f r$-Gen [92] kodiert für eine Methylase, die Kreuzresistenz gegen mehrere Antibiotika vermittelt (Phenicole, Oxazolidinone, Lincosamide, Pleuromutiline, Streptogramin A). Eine Weitergabe dieser Resistenzplasmide auf verwandte und nicht-verwandte Stämme über Spezies- und Gattungsgrenzen hinweg ist belegt $[93,94]$. So wurde das $c f r-G e n$ auch in Enterokokken nachgewiesen, wobei mehrere Allele vorkommen können. In Enterokokken wird allerdings die Rol- 
le von sowohl $c f r$ als auch $c f r(B)$ bei der Ausprägung der Linezolid-Resistenz kontrovers diskutiert $[95,96]$.

Die Erfassung der Resistenz mit derzeit kommerziellen Laborautomaten erfolgt zuverlässig; eine zweite, alternative Methode zur Überprüfung der Resistenz ist vorteilhaft, aber nicht zwingend, da die Vorhersage aus Methoden der Mikrobouillonverdünnung zuverlässig erfolgt und die Resistenz stabil ist [97].

Der Anteil der Linezolid-resistenten Isolate korreliert in gewisser Weise mit einem zunehmenden Einsatz der Substanz. Ansteigende Trends werden allerdings nur in Referenzlaboren auffällig [97]; in klassischen Resistenz-Surveillance-Systemen wie ARS [78] und den Studien der Paul-Ehrlich-Gesellschaft ist der Anteil Linezolid-resistenter Enterokokken-Isolate seit Jahren konstant $<1 \%$ [79].

\subsubsection{Tigecyclin}

Tigecyclin ist ein Tetracyclin-Antibiotikum mit bakteriostatischer Wirkung und sehr eingeschränkter Empfehlung für den Einsatz bei Enterokokkeninfektionen. Resistenzen in Enterokokken sind bisher selten, die zugrundeliegenden Mechanismen sind komplex und multifaktoriell.

Tigecyclin ist ein Tetracyclin-Antibiotikum mit einer zusätzlichen Seitenkette am Molekül und damit einer Wirksamkeit auch gegenüber Tetracyclin-resistenten Bakterien [98, 99]. Es ist das bisher einzige am Markt verfügbare Glycylcyclin. Es wirkt bakteriostatisch.

Für den Einsatz von Tigecyclin bei Bakteriämie und/oder Sepsis durch VRE liegen nur begrenzt Daten und Empfehlungen vor. Problematisch bei der Substanz ist, dass die erreichten Serumspiegel für die Therapie einer Sepsis nicht ausreichend hoch sind. In bestimmten Konstellationen wurde Tigecyclin erfolgreich als Kombinationspartner mit anderen Antibiotika wie Carbapenemen oder Daptomycin bei Fällen von schwerer Sepsis eingesetzt [100-102]. Es ist zugelassen für die Behandlung von komplizierten Haut- und Weichteilinfektionen sowie komplizierten intraabdominellen Infektionen und auch nur für diese Therapien existieren Grenzwerte bei EUCAST. Bei CLSI hingegen gibt es keine Grenzwerte für Tigecyclin bei
Enterokokken. Tigecyclin sollte bei invasiven VRE-Infektionen nur nach Abwägung möglicher Therapiealternativen und/oder als Kombinationspartner als letzte Option bei gleichzeitigem Monitoring von Nebenwirkungen in Betracht gezogen werden [103, 104].

Unempfindlichkeit gegen Tigecyclin bei grampositiven Krankenhauserregern, speziell Enterokokken, ist bisher sehr selten [105-107]. Es werden verschiedene genetische Veränderungen in Enterokokken diskutiert, welche im Zusammenhang mit Tigecyclin-Resistenz stehen können. Möglicherweise ist der Mechanismus auch multifaktoriell.

Bei Verdacht auf Tigecyclin-Unempfindlichkeit ist die Verwendung von wenigstens zwei unabhängigen, phänotypischen Testverfahren zu empfehlen. Die Stämme sollten zur Überprüfung an ein Referenzlabor geschickt werden.

\subsubsection{Daptomycin}

Daptomycin ist ein Lipopeptid-Antibiotikum. In den zugelassenen Dosierungen ist die Substanz nicht gegen Enterokokken wirksam. Resistenzen in Enterokokken sind sehr selten, die zugrundeliegenden Mechanismen schließen Mutationen in Genen des Zellmembranstoffwechsels, der Membranzusammensetzung und des -Ladungspotenzials ein. Das Auftreten von Daptomycin-resistenten Enterokokken korreliert mit einem erhöhten Einsatz der Substanz in betroffenen Einrichtungen.

Daptomycin ist ein zyklisches Lipopeptid. Es wirkt gegen die (innere) bakterielle Zellmembran und somit ausschließlich gegen grampositive Bakterien [108]. Daptomycin lagert sich in die Membran ein und verursacht einen Efflux von Kalium-Ionen, was zur Destabilisierung der Membran und Änderung des Ladungspotenzials führt. Unter optimalen Bedingungen ist die Wirkungsweise bakterizid. Laut Einschätzung von EUCAST (Stand 2018) hat Daptomycin in den zugelassenen Dosierungen keine nachgewiesene Wirksamkeit gegen Infektionen mit Enterokokken und folglich existiert auch kein klinischer Grenzwert (Status „IE = insufficient evidence"). Bei CLSI existiert ein Grenzwert für die Sensibel-Kategorie (sensibel $\leq 4 \mathrm{mg} / \mathrm{L}$ ) mit der Ausnahme, dass dieser nicht für Isolate aus dem respiratorischen Trakt gilt.

Es gibt ein Empfehlungsschreiben von EUCAST zur Verwendung von Daptomycin bei Enterokokken-Endokarditis [109]. Hierin wird der Stellenwert mit höheren Dosierungen von Daptomycin zur Behandlung einer Enterokokken-Endokarditis diskutiert. Analysen einer klinischen Anwendung dokumentieren variable Erfahrungen, auch und vor allem bei Enterokokken-Endokarditis [110]. Aktuelle Artikel beschreiben ein Versagen einer Daptomycin-Therapie in Abhängigkeit von der MHK und empfehlen eine Anpassung des Grenzwerts aus mikrobiologischer Sicht [111].

Insgesamt wird der Stellenwert von Daptomycin ggf. auch in Kombination mit Beta-Lactam-Antibiotika zur Behandlung von Enterokokken-Infektionen derzeit kontrovers diskutiert [112-115].

Enterokokken mit erhöhter MHK gegen Daptomycin sind vor allem im angloamerikanischen Raum nachgewiesen und intensiver bearbeitet worden. Mehrere Faktoren, die mit der Zusammensetzung der Zellmembran, dem Ladungspotenzial und der Fluidität assoziiert sind, stehen im Zusammenhang mit der Ausbildung und Selektion von Resistenzen unter Daptomycin-Therapie [116-119]. Alle diese Modifikationen beeinflussen im weitesten Sinne die bakterielle Zellwand- und Zellmembranhomöostase und verändern somit die Effektivität von Daptomycin.

Das NRZ hat bisher nur wenige Enterokokken mit Daptomycin-Unempfindlichkeit erhalten, in welchen die Unempfindlichkeit mit zwei unabhängigen Methoden bestätigt werden konnte [97]. Allerdings ist bekannt, dass in Einrichtungen mit einem häufigen Einsatz der Substanz Daptomycin-resistente Enterokokken selektiert werden [120]. Nicht selten kann eine ursprünglich angezeigte Unempfindlichkeit gegen Daptomycin in nachfolgenden Tests nicht bestätigt werden, was an einem instabilen Resistenzphänotyp liegen kann und nicht zwangsläufig auf eine fehlerhafte Diagnostik zurückzuführen ist. 


\subsection{Bedeutung der Enterokokken als Infektionserreger}

\subsubsection{Pathogenität}

Enterokokken werden bei Infektionen oft als Teil einer Mischflora nachgewiesen; bei unkomplizierten Infektionen kann häufig auf eine gezielte Therapie der Enterokokken verzichtet werden. Enterokokken-bedingte Infektionen bei Intensivtherapie- und immunsupprimierten Patienten, insbesondere nach Lebertransplantation, können hingegen mit einer hohen direkten Letalität einhergehen.

Enterokokken gelten als wichtiger Bestandteil der menschlichen Mikrobiota. Wenn jedoch durch Antibiotika, wie z. B. Metronidazol, die anaerobe Flora im Darm gehemmt wird, kann es im Darm zu massiver Proliferation von Enterokokken mit Translokation in tiefere Gewebe und in die Blutbahn mit Auslösung von Erkrankungen kommen [121-124]. Auch durch exogenen Eintrag von Enterokokken z. B. in Wunden und die Harnwege können Infektionen verursacht werden.

Bei der Diagnostik von Harnwegsinfektionen finden sich Enterokokken, insbesondere bei der Untersuchung von Mittelstrahlurin, häufig in einer Mischflora. In einer Studie an immunkompetenten jungen Frauen mit unkomplizierter Zystitis wurde gezeigt, dass es sich in der untersuchten Population in der Regel um eine Kontamination handelte [125].

Bei intraabdominellen Infektionen werden Enterokokken häufig nachgewiesen, meist als Teil einer Mischflora. Die pathogenetische Bedeutung ist daher im Einzelfall oft unklar. Wahrscheinlich ist ihre Rolle bei Patienten in gutem Allgemeinzustand und einfacheren Infektionen wie Appendizitis perforata oder Salpingitis als ursächliches Pathogen begrenzt; häufig wird auf die gezielte Therapie der Enterokokken in diesem Kontext verzichtet. Zunehmende Bedeutung erlangen jedoch Enterokokken-bedingte Infektionen bei Intensivtherapie-pflichtigen und immunsupprimierten Patienten, insbesondere nach Lebertransplantation [126]. Besonders Enterokokken-Bakteriämien gehen mit einer hohen direkten Letalitätsrate von bis zu $30 \%$ einher und treten wie andere Enterokokkeninfekti- onen vor allem bei älteren und immunsupprimierten Patienten auf [1]. In einer Studie wurde die Inzidenzrate der Enterokokkenbakteriämie unter Patienten einer Intensivstation mit 3,0 Fällen/1000 Patiententage unter Risiko angegeben, wobei die meisten Infektionen mit einem zentralvenösem Katheter assoziiert waren [127]. Die betroffenen Patienten wiesen eine erhöhte Letalität auf, die allerdings in der untersuchten Population nicht höher war als die Letalität der katheterassoziierten Sepsis durch Koagulase-negative Enterokokken.

Bei schwerkranken Patienten in schlechtem Allgemeinzustand mit komplexen Infektionen, wie multiplen Abszessen, Anastomoseninsuffizienzen, Cholangitiden mit Leberabszessen, abszedierenden Pankreatitiden ist der Nachweis von Enterokokken mit erhöhter Morbidität und Letalität verbunden, was bei der Therapie berücksichtigt werden sollte $[128,129]$.

Bisweilen wurde davon ausgegangen, dass Enterokokken zu häufigen Kontaminanten von Blutkulturen gehören, insbesondere, wenn sie nur in einer von mehreren Kulturen nachgewiesen wurden. Eine Untersuchung von 206 bakteriämischen Episoden durch Enterokokken zeigte jedoch, dass die Zahl positiver Blutkulturen nicht mit dem klinischen Outcome der Episode korreliert [130], das heißt, ein einmaliger Nachweis von Enterokokken in der Blutkultur kann genauso mit einer Infektion assoziiert sein wie ein mehrfacher Nachweis.

\subsection{2 Übertragung}

Enterokokken können direkt oder indirekt über die Hände des Personals, aber auch direkt durch Patienten und ebenso über kontaminierte Oberflächen übertragen werden. Enterokokken werden häufig in der unbelebten Umgebung nachgewiesen. An Untersuchungen von VRE-besiedelten Patienten zeigt sich, dass der Anteil von Kontaktpatienten, die besiedelt werden, hoch zu sein scheint (3-10 \%).

Enterokokken gehören zu den Mikroorganismen, für die mittels Typisierungen auch im endemischen Setting Übertragungen mit der Folge von nosokomialen
Infektionen nachgewiesen wurden [131, 132].

Weitere Daten zur Übertragbarkeit von Enterokokken ergeben sich aus Untersuchungen von VRE, da die Resistenz hier als Marker zur Verfügung steht. Im Vergleich verschiedener multiresistenter Mikroorganismen wurde gezeigt, dass für VRE häufiger ein nosokomiales Erwerben nachgewiesen werden konnten als für alle anderen multiresistenten Mikroorganismen [133], so dass die Autoren daraus schließen, dass der nosokomiale Nachweis von VRE zumeist durch nosokomiale Übertragungen bedingt ist.

Der Anteil von Kontaktpatienten zu VRE-Patienten, die positiv auf VRE gescreent wurden, wurde mit ca. 3-10 \% befunden $[134,135]$. Bettnachbarn von neu identifizierten VRE-Trägern können in ca. 10-20 \% ebenfalls VRE erwerben [136, 137]. Austin et al. errechneten für die Patienten einer ITS eine Reproduktionszahl von 3,8 , d. h. jeder VRE-Patient führte zur Übertragung auf drei bis vier weitere Patienten [138].

Als Wege der Übertragung werden neben der direkten Übertragung zwischen zwei besiedelten oder infizierten Personen, die indirekte Übertragung durch die Hände des Personals und die Übertragung durch kontaminierte Oberflächen diskutiert.

In Ausbruchsituationen wurde gezeigt, dass die Hände der Mitarbeiter eine wichtige Rolle für die Übertragung von Enterokokken spielen [139], was insbesondere für VRE wiederholt nachgewiesen wurde [140-145]. Nur in einer einzelnen Studie wurde gezeigt, dass ein Mitarbeiter mit intestinaler Kolonisation mit VRE zur Verbreitung des Erregers im Krankenhaus beigetragen hat [146].

Enterokokken werden leicht in die Umgebung von Patienten freigesetzt [147]. So wurden wiederholt Reservoire in der Umgebung als Quelle für Ausbrüche nosokomialer Infektionen mit Enterokokken nachgewiesen, z. B. Beatmungsschläuche [148], kontaminierte Inkubatoren [149], Griffe von Thermometern $[150,151]$ und andere kontaminierte Oberflächen [152, 153]. Die Bedeutung der Umgebungskontamination zeigt sich auch darin, dass sich nach Neuaufnahme in Patientenzimmer, die zuvor mit Trägern von VRE belegt wa- 
ren, ein erhöhtes Risiko für den Erwerb von VRE ergab [154, 155].

Enterokokken wurden sowohl in der patientennahen Umgebung auf der Toilette [156], auf Gegenständen wie Thermometer, Instrumente, Handschuhe als auch auf Griffkontaktflächen wie Türklinke, Bettenholm, Nachttisch, Telefon, Lichtschalter, PC-Display, Wasserhahn sowie auf dem Fußboden nachgewiesen [157]. Häufig zeigt sich jedoch eine hohe Umgebungskontamination, ohne dass ein direkter Bezug zu konkreten Infektionsfällen hergestellt werden konnte, z. B. auf Touchscreens von Computern [158], Computer-Tastaturen [159], Mobiltelefonen und Smartphones [160] oder Aufzugknöpfen [161].

Von den kontaminierten Flächen oder der Haut des Patienten können Enterokokken über die Hände bzw. über die behandschuhte Hand des Personals auf weitere Oberflächen übertragen werden. In Transferbeobachtungen zu VRE wurden die Enterokokken in $11 \%$ der Beobachtungen von besiedelten Patienten über Hände auf reine Flächen übertragen [139]. Nach Berührung des Patienten und seiner Umgebung waren 75 \% der bloßen Hände und nach Ablegen von Handschuhen noch $9 \%$ der Hände des Personals mit VRE kontaminiert. Wurden nur kontaminierte Flächen berührt, waren $21 \%$ bzw. 0 \% der Hände kontaminiert [162].

Bei Felduntersuchungen im Rahmen der Pflege von Patienten rangierten VRE nach multiresistenten Acinetobacter baumannii und Pseudomonas aeruginosa an dritter Stelle in der Übertragungshäufigkeit auf Kittel bzw. Handschuhe [163].

\subsubsection{Kolonisation}

Wie viele Enterokokken zur Entstehung einer Kolonisation mit antibiotikaresistenten Enterokokken notwendig sind, ist unbekannt. Eine geringe Erregermenge scheint jedoch auszureichen. Als Risikofaktoren für eine Kolonisation sind schwere Komorbidität, insbesondere Immunsuppression und hämatologische Krankheiten, vorausgegangene Antibiotikatherapie und vorausgegangene (längere) Hospitalisierung bekannt.

Da Enterokokken zur normalen intestinalen Flora gehören, wurden Untersuchungen zu Risikofaktoren für eine Be- siedelung nur für antibiotikaresistente Enterokokken durchgeführt.

Die Dosis für den Beginn einer intestinalen Kolonisation mit VRE ist unbekannt, vermutlich aber gering, weil die Restkontamination mit VRE nach Schlussdesinfektion des Patientenzimmers für nachfolgend aufgenommene Patienten das Kolonisationsrisiko erhöhte [154]. Bei Mäusen war allerdings nach oraler Applikation von $5 \times 10^{8} \mathrm{KBE}$ Vancomycin-resistente E. faecium nur eine transiente Kolonisation nachweisbar [164]. Als Infektionsdosis von VRE sind in Tiermodellen $<10^{3} \mathrm{KBE}$ beschrieben [165].

\section{Risikofaktoren für eine Besiedelung mit} Enterokokken. Patienten mit schwerer Komorbidität, insbesondere Immunsuppression und hämatologischen Krankheiten, haben ein erhöhtes Risiko, mit VRE kolonisiert oder infiziert zu werden [166, 167]. Eine Punkt-Prävalenz-Studie bezüglich VRE an einer Universitätsklinik ergab, dass vorangegangene Hospitalisierung, chronisches Nierenversagen sowie ein langer Krankenhausaufenthalt mit VRE-Besiedelung assoziiert waren [167, 168].

Eine Untersuchung in einem endemischen Setting zeigte für eine Kohorte von Patienten bei Aufnahme in eine Rehabilitationsklinik, dass Diabetes mellitus, eine Pneumonie in der Anamnese und die Therapie mit Cotrimoxazol Risikofaktoren für die Besiedelung mit VRE waren [169].

Vorausgehende Therapie mit Antibiotika war Gegenstand mehrerer Studien mit widersprüchlichen Ergebnissen [170, 171]. Eine frühere Therapie mit Cephalosporinen der dritten Generation, Metronidazol und Fluorchinolonen wurde als Risikofaktor für die VRE-Kolonisierung identifiziert, nicht jedoch eine Behandlung mit Vancomycin [170, 172]. Allerdings konnten viele der klinischen Studien aufgrund ihres Designs (retrospektiv, Monocenter, kleine Stichprobengröße) in systematischen Untersuchungen nicht zur Kontrolle herangezogen werden [173].

Für den Erwerb von VRE im Krankenhaus wurden bereits zu Beginn der Verbreitung von VRE Modelle entwickelt, in denen der Zusammenhang zwischen Prävalenz von VRE-Trägern (Kolonisations- druck) und der Neubesiedelung von Patienten mit VRE untersucht wurde [157]. Ein Review aus dem Jahr 2011 zeigte, dass Studien, in denen ein Zusammenhang zwischen Kolonisationsdruck und Neuerwerb multiresistenter Erreger untersucht wurde, aufgrund verschiedener Definitionen des Kolonisationsdruckes (z. B. Prävalenz oder Inzidenzdichte von Patienten mit multiresistenten Erregern) nur schwer vergleichbar sind. Dennoch zeigten zehn von 13 Studien eine unabhängige Assoziation zwischen Kolonisationsdruck und Erwerb von VRE [173].

Für Patienten bei Aufnahme in ein Krankenhaus untersuchten Tacconelli et al. an zwei US-amerikanischen Universitätskliniken mit einer hohen endemischen VRE-Rate die Risikofaktoren für eine Besiedelung mit VRE, um einen Risikoscore zu erstellen, mit dem VRE-Träger bereits bei Aufnahme identifiziert werden können [174]. Auf Basis der unabhängigen Risikofaktoren und deren assoziierter Odds Ratio wurden folgende Komponenten für einen Score vorgeschlagen:

- MRSA-Besiedelung in den vorangegangenen 12 Monaten 4 Punkte

- Langzeit-Dialyse 3 Punkte

- Übernahme aus einem Pflegeheim

- Einnahme von mehr als einem Antibiotikum in den letzten 30 Tagen 3 Punkte

Krankenhausaufenthalt in den vorangegangenen 12 Monaten

- Alter höher als 60 Jahre

3 Punkte

2 Punkte

In Abhängigkeit vom Cut-off für den Score lag die Sensitivität bei 44 \% (Spezifität $98 \%$ ) bei einem Cut-off von $>9$ Punkten und bei $75 \%$ (Spezifität $92 \%$ ) bei einem Cut-off von $>6$ Punkten für die Detektion von VRE-Trägern.

VRE bei Mitarbeitern im Krankenhaus. Es existieren nur wenige Daten zur Besiedelung von Mitarbeitern, welche in der Pflege oder Behandlung von VRE-Patienten beteiligt sind. Decker et al. fanden in einer Kohorte von nahezu 400 Mitarbeitern, die Kontakt zu Patienten mit multiresistenten Erregern, darunter auch VRE, hatten, keinen Mitarbeiter bei dem VRE 
nachgewiesen werden konnte [175]. Dagegen stellten Baran et al. eine häufigere Besiedelung bei Mitarbeitern mit Kontakt $\mathrm{zu}$ VRE-Patienten fest (2 von 52) als bei solchen ohne Kontakt (0 von 40) [176]. In zwei Fällen konnte eine Weitergabe an Haushaltskontakte der VRE-Träger gezeigt werden [176].

\subsubsection{Dauer der Besiedelung}

Die Dauer einer gastrointestinalen Besiedlung mit VRE kann lang sein, wobei zu berücksichtigen ist, dass derzeit keine verlässlichen Kriterien bzw. Methoden für die Beurteilung der VRE-Freiheit definiert sind.

Auch die Dauer einer Besiedelung wurde nur an VRE untersucht, da Enterokokken zur normalen intestinalen Flora gehören. VRE können den Gastrointestinaltrakt lang anhaltend besiedeln. Als Kolonisationsdauer sind Zeiträume von Wochen bis zu mehr als drei Jahren beschrieben [177-179].

In einer Meta-Analyse von 12 Kohortenstudien zur Dauer der Kolonisation mit VRE fand sich eine erhebliche Heterogenität u.a. auch in der Definition von VRE-Freiheit und der Dauer der Nachbeobachtung [180]. Der Anteil der jeweiligen Patientenkohorten, für die VREFreiheit nachgewiesen wurde, reichte von $0 \%$ bis über $80 \%$. Die Besiedelungsdauer bis zur VRE-Freiheit reichte von einer bis 43 Wochen [180]. Nach Entlassung aus der Klinik betrug die mittlere Zeit, in der VRE noch kulturell nachgewiesen werden konnten, ca. sechs Wochen mit einer Spanne von 0-50 Wochen [181]. Risikofaktoren für ein verlängertes Trägertum waren chirurgische Eingriffe, Antibiotikaeinnahme während des Krankenhausaufenthaltes, Dialyse und Entlassung in ein Altenheim oder eine andere Gesundheitseinrichtung [181]. In einer retrospektiven Studie wurde als längste Dauer für ein Trägertum 46,5 Monate ermittelt [182]; allerdings kann aufgrund der Erhebungsmethode eine Rekolonisation nicht ausgeschlossen werden.

Ausschließlich ambulant betreute, mit VRE kolonisierte, aber nicht antibiotisch behandelte Kinder mit onkologischer oder hepatologischer Grunderkrankung oder Zystischer Fibrose schieden VRE im Mittel sechs Wochen aus [183]. Bei päd- iatrisch-onkologischen Patienten betrug die mittlere Ausscheidungsdauer 12-16 Wochen [183-186]. In einer Gruppe von Kindern, die VRE im Rahmen eines Ausbruchs auf einer neonatologischen ITS erworben hatten, war die mittlere Besiedlungsdauer 206 Tage (144-276 Tage) [187].

Bei einem kleinen Teil der Patienten können VRE während eines Krankenhausaufenthaltes nicht mehr nachweisbar sein. Ghosh et al. fanden unter Patienten mit einer Aufenthaltsdauer von mehr als 30 Tagen bei wöchentlichen Abstrichen, dass für $18 \%$ der VRE-Patienten nach im Mittel 26 Tagen ein VRE-Nachweis nicht mehr möglich war [188].

\subsubsection{Nosokomiale Infektion}

Enterokokken gehören zu den am häufigsten nachgewiesenen Bakterien bei nosokomialen Wund- und Harnweginfektionen und der katheterassoziierten Sepsis, wobei hierbei der Anteil von E. faecium stark zugenommen hat. Je nach Risikopopulation können 10-20 \% der kolonisierten Patienten eine Infektion erleiden. Risikofaktoren für eine Infektion sind Komorbidität, bestehende Kolonisation, ZVK und vorausgegangene Antibiotikatherapie. Am häufigsten treten schwerwiegende Infektionen in der Hämatologie-Onkologie und auf Intensivstationen auf.

Enterokokken gehören zu den am häufigsten aus klinischen Materialien von Krankenhauspatienten nachgewiesenen Spezies. Nach E. coli und C. difficile sind E. faecium und E. faecalis und Clostridioides (früher Clostridium) (C.) difficile zusammen die dritthäufigsten Erreger von NI in Deutschland $[189,190]$ und die dritt- bis vierthäufigsten Erreger weltweit [191], wobei E. faecium zunehmend an Bedeutung gewinnt. In den letzten Jahren ist der Anteil von E. faecium im Vergleich zu E. faecalis stetig gestiegen [192]. In den Antibiotikaresistenz-Studien der PaulEhrlich-Gesellschaft für Chemotherapie e. V. (PEG) zeigte sich ein Ansteigen des Anteils der E. faecium-Isolate (bezogen auf alle untersuchten Enterokokken-Isolate) von 9,3\% (1998) über 24,4 \% (2004) auf 43,0 \% (2013) [6, 193-201].

In Erregerstatistiken des OP-KISS aus 2016 sind Enterokokken als Erreger bei
$17,8 \%$ aller postoperativen Wundinfektionen aufgeführt und dabei in $6 \%$ alleiniger nachgewiesener Erreger, wobei der Anteil bei abdominalchirurgischen Eingriffen erwartungsgemäß am höchsten war (nachgewiesen bei $30 \%$ aller postoperativen Wundinfektionen) [202]. In einer Analyse mit ausschließlicher Berücksichtigung kolorektaler Resektionen, wobei in $10 \%$ der Fälle randomisiert retrospektiv zusätzlich die Patientenakten überprüft wurden, betrug der Anteil von E. faecalis und $E$. faecium bei postoperativen Wundinfektionen 23,8 \% bzw. 23,6\%, bei Peritonitis $16,1 \%$ bzw. 13,2 \% und bei laparoskopischem Revisionseingriff 25,4 \% bzw. $39,1 \%$. Der Anteil von Enterokokken als alleiniger Erreger betrug 9,0 \%, 4,6 \% bzw. $11,7 \%$ [203].

Auf Intensivstationen, die sich am KISS-System beteiligen, waren Enterokokken die zweithäufigsten Erreger der katheterassoziierten Sepsis (20\%) und der katheterassoziierten Harnwegsinfektion (bei $27 \%$ aller Harnwegsinfektionen) [204].

Bei Endokarditis und late-onset Infektion nach Implantation künstlicher Herzklappen wurden Enterokokken in $20 \%$ bis etwa $30 \%$ der Fälle nachgewiesen [205, 206]. Auch bei Gefäßprothesen [207], ventrikulo-peritonealem Shunt [208], externen Liquorableitungssystemen und intraokularen Linsen [209] wurden Enterokokken als ätiologisch relevante Erreger nachgewiesen einschließlich einer Zunahme von VRE [210, 211].

Hingegen gelten Enterokokken nicht als Auslöser von respiratorischen Infektionen. Der Nachweis in respiratorischen Sekreten entsteht durch Verschleppung oropharyngealer Standortflora und hat keine therapeutischen Konsequenzen [212].

Häufigkeit des Auftretens von Infektionen bei besiedelten Personen. Der $\mathrm{Zu}$ sammenhang zwischen Kolonisation und Infektion wurde nur für VRE untersucht, da Enterokokken zur Normalflora des Menschen gehören. Nur ein verhältnismäßig kleiner Anteil von Patienten mit VRE-Kolonisation erleidet eine Infektion. Hierbei schwankt der Anteil von Patienten mit symptomatischen Infektionen in Abhängigkeit von der spezifischen $\mathrm{Pa}$ tientenpopulation im Bereich von $8 \%$ bei 
pädiatrischen Onkologiepatienten [184] und $24 \%$ bei hämato-onkologischen $\mathrm{Pa}$ tienten [213], während das Risiko bei gesunden Individuen gering ist [214].

Der Anteil VRE-kolonisierter Patienten, der während des Aufenthaltes in einer Einrichtung eine Infektion erleidet, wurde mit 14-20 \% beschrieben [135, 215-217], wobei für die Definition einer Infektion in der Regel die CDC-Kriterien genutzt wurden. Datta et al. untersuchten 199 VRETräger in einer retrospektiven Kohortenstudie über einen Krankenhausaufenthalt hinaus. Von 199 eingeschlossenen Patienten entwickelten $8 \%$ Infektionen in den 18 Monaten nach Erstnachweis von VRE, von denen zehn Infektionen nach Krankenhausentlassung auftraten. In zwei Fällen handelte es sich um Bakteriämien und in drei Fällen führte die Infektion zur erneuten Krankenhausaufnahme [218].

Risikofaktoren für Infektionen. Risikofaktoren für das Auftreten von Enterokokken-Infektionen wurden nahezu ausschließlich im Rahmen von Infektionen durch antibiotikaresistente Enterokokken untersucht. Für Bakteriämien durch HL-GRE fanden sich chronische Niereninsuffizienz, Behandlung auf einer Intensivstation und vorangegangene Antibiotikatherapie als unabhängige Risikofaktoren [219].

$\mathrm{Zu}$ Risikofaktoren, die mit dem Auftreten von Infektionen durch VRE verbunden sind, gehören patienteneigene Risikofaktoren wie Niereninsuffizienz, Chemotherapie, verminderte Neutrophilenzahl, länger andauernde Neutropenie, hoher APACHE II-Score, Komorbidität, Alter über 60 Jahre, vorangegangene antibiotische Therapie, insbesondere mit Vancomycin und Kolonisation des Darmtraktes mit VRE [157, 220-225]. Risikofaktoren für eine Bakteriämie mit VRE stellen auch Hämodialyse, die Behandlung mit Kortikosteroiden oder Zytostatika, eine totale parenterale Ernährung, chirurgische Eingriffe, Neutropenie und Mukositis dar [226-228].

Einfluss der Art der Grunderkrankung. Als Risikofaktoren für VRE-Infektionen sind schwere Begleiterkrankungen (z.B. Nierenversagen und Karzinome) [166] und eine längere Dauer des Krankenhaus- aufenthaltes bekannt. Morris et al. fanden in einer Universitätsklinik die höchste Rate an VRE-Infektionen auf einer Transplantationsstation (13,2 Infektionen/1000 Aufnahmen), gefolgt von Intensivstationen mit 4,8-5,6 Infektionen/1000 Aufnahmen. Auf internistischen Stationen lag die Infektionsrate bei 1,8/1000 Aufnahmen [229]. Bei pädiatrischen Patienten lag die höchste Besiedelungs- und Infektionsrate auf hämatologisch-onkologischen Stationen [224, 230].

Von Ausbrüchen durch Enterokokken waren entsprechend am häufigsten Abteilungen der Inneren Medizin (32\%, $n=151$ ) betroffen, gefolgt von Hämatologie/Onkologie (29\%), Chirurgie (26\%), Hämodialyse (11\%), Transplantation (10\%) und Neonatologie (9\%)(Outbreak database, Stand 8. Januar 2018, 3529 Einträge in der Datenbank) [231].

Auch die Behandlung von C. difficileInfektionen kann durch das Unterdrücken der anaeroben Darmflora vermehrtes Wachstum von Enterokokken mit Translokation in tiefere Gewebe und in die Blutbahn und somit nachfolgende Infektionen begünstigen [232, 233].

Bedeutung der vorausgegangenen KoIonisation. Mit VRE kolonisierte Patienten weisen eine hohe Prävalenz der Hautkolonisation mit VRE auf, die das Risiko einer Gefäßkatheter-assoziierten Sepsis erhöhen kann. In Untersuchungen bei pädiatrischen Patienten fanden die Autoren, dass bei einer VRE-Kolonisation das Risiko für die VRE-Infektion signifikant (bis zu 9-fach) erhöht war [224, 234]. Patienten, bei denen VRE aus Blut, Urin oder Wunden isoliert wurde, haben zudem eine große Anzahl von VRE in Stuhlproben [121].

Einfluss der Antibiotikatherapie. Ebenfalls ein wichtiger Risikofaktor war eine der Infektion vorausgegangene Antibiotikaexposition. Eine Studie ergab, dass sich die Disposition für eine VRE-Infektion durch die Antibiotikaexposition gegenüber Vancomycin zwischen 2,3- und 11,0-fach erhöhte [235].

In einer prospektiven Studie bei $\mathrm{Pa}$ tienten auf der Intensivstation wurden Verabreichung von Cephalosporinen der dritten oder vierten Generation, Corti-
son-Verwendung vor der ITS-Aufnahme und VRE-Kolonisation als Risikofaktoren für Enterokokken-Infektion identifiziert, wohingegen enterale Ernährung ein schützender Faktor war [236]. Bei Personen mit VRE-Kolonisation förderten Antibiotika mit Wirksamkeit gegen anaerobe Bakterien die Dichte der Besiedelung, während Antibiotika mit minimaler Aktivität gegenüber anaeroben Bakterien keinen Einfluss hatten [121].

\subsection{Outcome von Enterokokken- Infektionen in Abhängigkeit von der Antibiotikaresistenz}

\subsubsection{Outcome bei Infektionen mit high-level Gentamicin resistenten Enterokokken}

Es scheint ein Zusammenhang zwischen Bakteriämie mit high-level Gentamicinresistenten Enterokokken und schwerwiegenderen komorbiden Bedingungen sowie einer höheren Letalität zu bestehen. Dabei scheint die high-level Gentamicin-Resistenz selbst nicht wesentlich zur Letalität beizutragen.

Kontrovers diskutiert wird die Frage, ob high-level Gentamicin-Resistenz die Prognose von Patienten mit Enterokokken-Bakteriämie beeinflusst. In einer israelischen Studie mit 117 Fällen mit VRE-Bakteriämie war die der Infektion zuzuschreibende Sterblichkeit $(19 \%)$ deutlich $(p<0,01)$ an zwei voneinander unabhängige Variablen geknüpft: Schweregrad der Krankheit $(\mathrm{OR}=39,6)$ und high-level Gentamicin-Resistenz $(\mathrm{OR}=6,4)$ [237]. Hierbei ist anzumerken, dass die Patienten mit Infektionen durch high-level Gentamicin-resistente Enterokokken unter ernsten Komorbiditäten litten und z. T. Ampicillin-resistente Enterokokken nachgewiesen wurden [237]. Gegenteiliges erbrachten zwei Studien aus den USA und Spanien, deren Daten zeigten, dass high-level Gentamicin-Resistenz nicht die unspezifische Mortalität bei Patienten mit Bakteriämie beeinflusst [219, 238]. Es ist anzumerken, dass das Patientenalter deutlich unter dem der vorigen Studie lag (58 bzw. 64 Jahre gegenüber 74 Jahre). Des Weiteren kamen in der single-center Studie, die einen Effekt von Gentamicin-Resistenz zeigte, im gleichen Zeitraum ungefähr gleich viele 
Bakteriämien zustande wie in den anderen Studien, welche Daten aus 13 Krankenhäusern bzw. über einen Zeitraum von fünf Jahren anstatt von einem Jahr zusammenstellen. Dieses könnte auf eine deutlich höhere Morbidität in der Patientenpopulation der zuerst genannten Studie hinweisen, was eine höhere Letalität erklären könnte.

In einer Studie jüngeren Datums wurden 136 Patienten mit durch HL-GRE verursachter Bakteriämie mit 79 Patienten in der Kontrollgruppe, die eine Bakteriämie durch Enterokokken erlitten, die nicht high-level Gentamicin-resistent waren [239]. Hämatologische Malignität, APACHE II-Score, Neutropenie, Infektionen mit E. faecium und nosokomiale Infektionen kamen in der Gruppe mit HLGRE häufiger vor (37\% bei HL-GRE vs. $15 \%$ bei nicht high-level Gentamicin-Resistenz, $p=0,001$ ). Die 14- und 30-TagesMortalität war in der monovariaten Analyse höher in der Verumgruppe als in der Kontrollgruppe (50\% vs. $22 \%, p<0,001$ ) [239]. Ein Cox-Regressions-Modell wurde angewendet, um voneinander unabhängige Prädiktoren von Mortalität zu definieren. Die Analyse identifizierte APACHE II-Score, Knochenmarktransplantation, Gebrauch von Kortikosteroiden und unangemessene Antibiotikatherapie, aber nicht die Infektion mit HL-GRE als unabhängige Risikofaktoren für eine 30-Tage-Mortalität [239]. Auch eine multivariate Analyse in einer retrospektiven Studie in Neuseeland erbrachte keinen Zusammenhang zwischen high-level Gentamicin-Resistenz und Mortalität [240]. Einschränkungen bei all diesen Studien sind geringe Stichprobengröße, retrospektives Design, z. T. monozentrisches Design sowie das Fehlen von molekularepidemiologischen Daten.

\subsubsection{Outcome bei Infektionen mit VRE}

Die Sterblichkeit bei VRE-Sepsis beträgt 20-50 \% und ist nach vorliegenden Meta-Analysen höher als bei VSESepsis, wobei aktuell nicht sicher geklärt ist, ob hierbei Unterschiede in der verursachenden Spezies (E. faecium vs. E. faecalis) eine Rolle spielen. Ob die Vancomycin-Resistenz unter den aktuellen Therapieregimen mit einer zusätzlichen erhöhten Sterblichkeit verbunden ist, bedarf weiterer Studien.

Für andere VRE-Infektionen wurde ein Unterschied in der Sterblichkeit nicht gezeigt, wobei hierzu nur einzelne kleine Studien vorliegen. Besonders von erhöhter Sterblichkeit betroffen sind Patienten nach Leber- bzw. Stammzelltransplantation.

Es liegt eine Reihe von Studien vor, die das Outcome von Kolonisation oder Infektion mit VRE untersuchten. Hierbei können die Studien gruppiert werden in solche, die nicht zwischen Kolonisation und/oder bestimmten Arten von Infektionen unterschieden, solchen die einzelne Arten von Infektionen untersuchten und solchen, die sich auf bestimmte Patientenpopulationen konzentrierten.

\section{Untersuchungen ohne Unterscheidung von Kolonisation und Infektion. In ei-} ner Gruppe von Patienten mit Kolonisation und verschiedenen Arten von Infektionen fanden Tripathi et al. eine doppelt so hohe Sterblichkeit unter Patienten mit $\operatorname{VRE}(31,4 \%)$ wie unter Patienten mit VSE $(16,1 \%)$ [241]. Hierbei handelt es sich jedoch nur um einen Vergleich von Gruppen ohne geeignete Kontrolle für andere Faktoren, wie z. B. der Grunderkrankung.

Outcome bei Patienten mit VRE-Kolonisation. In einer gepaarten Fall-Kontroll-Studie, in der 199 VRE-kolonisierte Patienten mit 199 nicht kolonisierten Patienten verglichen wurden, fanden sich für die kolonisierten Patienten eine erhöhte Sterblichkeit, ein längerer Krankenhausaufenthalt und erhöhte Kosten [242].

Outcome bei Patienten mit VRE-Infektionen. Eine retrospektive gepaarte FallKontroll-Studie einer deutschen Universitätsklinik zeigte keinen signifikanten Unterschied in der Sterblichkeit von 42 Patienten mit VRE-Infektionen zu $42 \mathrm{~Pa}-$ tienten mit VSE-Infektionen, wobei keine Daten zur Art der Infektionen aufgeführt wurden. In dieser Studie wurde eine Vielzahl von Kriterien für die Zusammenstellung der Paare herangezogen, primär mit dem Ziel einen Vergleich der Kosten der Infektion zu erreichen [243]. Kosten für Patienten mit VRE-Infektionen lagen signifikant höher als Kosten für Patienten mit
VSE-Infektionen, was vor allem durch höhere Medikamentenausgaben bedingt war [243].

Outcome bei Sepsis und/oder Bakteriämie. Am häufigsten untersucht wurden die Folgen der Nachweise von VRE in Blutkulturen (Bakteriämien oder Sepsis). 2005 publizierten DiazGranados et al. eine Meta-Analyse von neun Studien mit 1614 Bakteriämien durch Enterokokken (683 VRE-Episoden und 931 VSE-Episoden). Sie fanden ein etwa zweifach erhöhtes Sterblichkeitsrisiko für Patienten mit VRE-Bakteriämien [1].

Es wurde vermutet, dass die höhere Sterblichkeit der VRE-Bakteriämie durch die fehlenden therapeutischen Möglichkeiten bedingt war. Neuere Studien und Meta-Analysen untersuchten daher die Sterblichkeit der VRE-Bakteriämie/Sepsis nach Einführung effektiver Antibiotika, zum Teil in Therapievergleichsstudien, zum Teil in speziellen Patientenpopulationen [244-248]. Eine aktuelle Meta-Analyse von 12 Kohorten und einer Fall-Kontroll-Studie kam zu dem Schluss, dass trotz effektiver zur Verfügung stehender Antibiotika eine VRE-Bakteriämie mit signifikanter Verlängerung des Krankenhausaufenthaltes und signifikant erhöhter Sterblichkeit einherging [248]. Zu dieser Meta-Analyse wurde kritisiert, dass nur ein Teil der eingeschlossenen Studien für Risikofaktoren adjustiert waren und nicht nach Spezies der Enterokokken gepaart wurde.

Outcome bei Harnwegsinfektionen. Für Harnwegsinfektionen und Bakteriurien durch Enterokokken ergab sich kein Unterschied zwischen Vancomycin-resistenten und Vancomycin-sensiblen Fällen hinsichtlich klinischer Präsentation (47\% asymptomatische Bakteriurie, $8 \%$ Zystitis, $25 \%$ Pyelonephritis und $20 \%$ nicht klassifizierte Bakteriurie) oder Outcome (Aufnahme auf eine ITS, Dauer des Krankenhausaufenthaltes oder Sterblichkeit) [249].

Outcome bei Infektionen des ZNS. Infektionen des ZNS durch VRE wurden in einer Fallserie von 38 Patienten in über $80 \%$ durch E. faecium ausgelöst [211]. Die Infektion war in mehr als der Hälfte der Fälle 
mit einem in situ befindlichen Device assoziiert. $18 \%$ der Patienten starben an der Infektion [211]. Eine weitere Fallserie von 39 Patienten, von denen 15 eine Infektion durch VRE hatten, zeigte eine Letalität von $21 \%$ [250]. In beiden Studien wurden keine direkten Vergleiche zwischen VRE und VSE durchgeführt.

Outcome bei VRE-Endokarditis. In einer Serie von 50 Patienten mit VRE-Endokarditis war die Hälfte der Fälle durch E. faecium verursacht. Bakteriämien durch E. faecium waren im Mittel mit 14 Tagen länger als durch $E$. faecalis verursachte Bakteriämien, welche im Mittel vier Tage dauerten. Die 30-Tage-Sterblichkeit unter den eingeschlossenen Patienten lag bei $38 \%$ und unterschied sich nicht signifikant zwischen E. faecium und E. faecalis [251]. Ein Vergleich mit der Sterblichkeit der VSEEndokarditis wurde nicht durchgeführt.

Outcome von Infektionen bei Transplantationspatienten. Eine besondere Bedeutung hat die Kolonisation oder Infektion mit Enterokokken bei Transplantationspatienten. Daten der Schweizer Organtransplantationskohorte zeigten, dass unter Transplantationspatienten die Lebertransplantation mit der höchsten Enterokokken-Infektionsrate verglichen mit Nieren-, Lungen- oder Herztransplantationen einhergeht [252].

Kim et al. verglichen Patienten, die vor Transplantation mit VRE besiedelt waren und Patienten, die nach Transplantation VRE erwarben mit Patienten, die nicht VRE-positiv waren. Sie fanden eine signifikant erhöhte VRE-Infektionsrate und Sterblichkeit bei Patienten, die nach der Transplantation VRE erwarben [253]. Eine Meta-Analyse kam zu dem Schluss, dass die VRE-Besiedelung bei Lebertransplantierten vor und nach Transplantation mit einem 6- bzw. 7-fach erhöhten Risiko für eine Infektion einherging [126].

In einer amerikanischen Kohorte von stammzelltransplantierten Patienten gehörten VRE zu den häufigsten Erregern der Sepsis [254]. Unter den NeutropeniePatienten verursachten VRE 31 Episoden, die ausschließlich als „break through“Bakteriämien unter antibiotischer Therapie auftraten. Ein septischer Schock trat bei $12 \%$ der VRE-Episoden auf, die 7-Tage-Sterblichkeit lag bei $18 \%$ [254].

Vydra et al. fanden eine erhöhte Sterblichkeit unter Patienten mit Enterokokken-Bakteriämie bei Stammzelltransplantationspatienten. Eine vorausgegangene Besiedlung mit VRE vor oder nach der Transplantation erhöhte das Risiko einer Bakteriämie um das 3- bzw. 7-fache. Die 30-Tage-Sterblichkeit lag bei $38 \%$, wobei es jedoch keinen Unterschied zwischen VRE und VSE gab [255]. Tavadze et al. postulierten in einer Studie zum Outcome von VRE-Bakteriämie unter allogen Stammzelltransplantierten, in der sie eine Gesamtsterblichkeit von über $88 \%$ (67 von 76 Patienten) unter den Patienten mit VRE-Sepsis beobachteten, dass die VRE-Sepsis nicht Ursache, sondern nur Anzeichen eines komplizierten Verlaufs sei [256]. Ähnlich interpretierten Hefazi et al. ihre Ergebnisse in einer Gruppe von allogen stammzelltransplantierten Patienten, in der sie eine erhöhte Rate von Bakteriämien unter VRE-Besiedelten fanden, jedoch keine erhöhte Sterblichkeit [221].

Outcome von Infektionen bei pädiatrischen Patienten. Von VRE-Infektionen in der pädiatrischen Patientenpopulation wird seltener berichtet als bei Erwachsenen. In den USA wurde seit 1997 ein signifikanter Anstieg von VRE-Infektionen beobachtet [257]. Pädiatrische Patienten mit VRE-Infektionen waren signifikant länger im Krankenhaus und verursachten höhere Kosten. Sie wiesen jedoch keine höhere Sterblichkeit auf als die Vergleichsgruppe [257].

Outcome in Abhängigkeit von der Enterokokken-Spezies. Der Einfluss der Enterokokken-Spezies wurde mehrfach untersucht [258-260]. Hierbei zeigte sich eine höhere Sterblichkeit der Vancomycin-resistente E. faecium-Infektionen. Bei einem Vergleich von 105 Patienten mit einer Bakteriämie durch Vancomycin-resistente E. faecalis und 197 Patienten mit einer Bakteriämie durch Vancomycin-resistente E. faecium zeigte sich für Vancomycin-resistente E. faecalis ein mehr als zweifach geringeres Risiko an der Bakteriämie zu versterben als bei Vancomycin-resistenten E. faecium [259]. In einer Untersuchung von Bakteriämien durch
Vancomycin-resistente E. gallinarum oder Vancomycin-resistente E. casseliflavus lag die 30-Tage-Sterblichkeit bei 10,4\% (5 von 48 Patienten), wobei die Behandlung mit Linezolid oder Daptomycin das Outcome signifikant verbesserte [261].

Outcome in Abhängigkeit vom Resistenztyp. Es konnten keine Studien identifiziert werden, in denen der Einfluss des Van-Typs (VanA versus VanB) auf das Outcome von Infektionen vergleichend untersucht wurde [222]. In den meisten Untersuchungen wurden keine Daten zur Art der Glykopeptid-Resistenz erhoben bzw. angegeben. Cheah et al. führten eine Fall-Kontroll-Studie zu Bakteriämien durch VanB-Typ VRE durch und fanden, dass VanB-Typ VRE im Vergleich zu VSE mit längerer Krankenhausverweildauer und höheren Kosten einherging. Es bestanden keine Unterschiede in der Sterblichkeit [222]. In einer Gruppe von 14 Patienten mit hämato-onkologischen Grunderkrankungen und Infektionen durch VanB-E. faecium fanden Worth et al. eine Gesamtsterblichkeit von $21 \%$, wobei sie nur in einem Fall (7 \%) einen direkten Zusammenhang mit der VRE-Sepsis sahen [262].

\subsubsection{Outcome bei Infektionen mit Linezolid-resistenten Enterokokken} Bei Infektionen durch LRE konnte im Vergleich zu LSE kein Unterschied bzgl. der Sterblichkeit gezeigt werden, wobei hierzu nur einzelne kleine Studien vorliegen und sich die Datenlage zumeist auf VRE mit zusätzlich bestehender Linezolid-Resistenz bezieht.

Der Einfluss der Linezolid-Resistenz bei Enterokokken auf die klinischen Outcome-Parameter der Patienten wurde in einigen retrospektiven Studien mit teilweise geringen Fallzahlen und unterschiedlichen Studiendesigns (Fall-Kontroll-Studien und Kohortenstudien) sowie heterogenen Patientenkollektiven untersucht. In fünf Studien wurden Patienten mit Linezolid-sensiblen VRE mit Patienten mit Linezolid-resistenten VRE verglichen [263-267], in einer weiteren war dies geplant [268].

In der endemischen Situation hatten Patienten mit LVRE keine erhöhte Sterblichkeit im Vergleich zu Patienten mit LVSE. Die Länge des Krankenhausauf- 
Tab. 4 Rationale Ableitung für die Durchführung erweiterter Hygienemaßnahmen bei Infektionen oder Besiedelung mit antibiotikaresistenten Enterokokken

\begin{tabular}{|c|c|c|c|c|c|}
\hline & $\begin{array}{l}\text { Verbreitung der resis- } \\
\text { tenten Stämme }\end{array}$ & $\begin{array}{l}\text { Reproduktion im } \\
\text { Krankenhaus }\end{array}$ & $\begin{array}{l}\text { Krankenhaus-assozi- } \\
\text { ierte Verbreitung in } \\
\text { Deutschland }\end{array}$ & Infektionsrate & $\begin{array}{l}\text { Gegenüber sensiblen } \\
\text { Isolaten erhöhte } \\
\text { Mortalität }\end{array}$ \\
\hline High-level Gentamicin & $\begin{array}{l}\text { Klonal und durch hori- } \\
\text { zontalen Gentransfer }\end{array}$ & $\begin{array}{l}\text { Unklar } \\
\text { (fehlende Daten) }\end{array}$ & Unbekannt & Bis $4,1 \%[284]$ & Nein \\
\hline $\begin{array}{l}\text { Vancomycin und } \\
\text { Teicoplanin (VanA) }\end{array}$ & $\begin{array}{l}\text { Klonal und durch hori- } \\
\text { zontalen Gentransfer }\end{array}$ & $\begin{array}{l}\text { Besiedelung bei } \\
3-10 \% \text { der Kontakte }\end{array}$ & HA-E. faecium & $\begin{array}{l}\text { Infektion bei 10-20\% } \\
\text { der Besiedelten }\end{array}$ & Bis zu zweifach erhöht \\
\hline Vancomycin (VanB) & $\begin{array}{l}\text { Klonal und durch hori- } \\
\text { zontalen Gentransfer }\end{array}$ & Vermutlich wie VanA & HA-E. faecium & Vermutlich wie VanA & Unklar, eher erhöht \\
\hline Linezolid & Überwiegend klonal & $\begin{array}{l}\text { Unklar } \\
\text { (fehlende Daten) }\end{array}$ & $\begin{array}{l}\text { Assoziation mit Einsatz } \\
\text { von Linezolid }\end{array}$ & $\begin{array}{l}\text { Unklar } \\
\text { (fehlende Daten) }\end{array}$ & $\begin{array}{l}\text { Unklar } \\
\text { (fehlende Daten) }\end{array}$ \\
\hline $\begin{array}{l}\text { Vancomycin und } \\
\text { Linezolid }\end{array}$ & Überwiegend klonal & $\begin{array}{l}\text { Unklar } \\
\text { (fehlende Daten) }\end{array}$ & $\begin{array}{l}\text { Assoziation mit Einsatz } \\
\text { von Linezolid }\end{array}$ & $\begin{array}{l}\text { Unklar } \\
\text { (fehlende Daten) }\end{array}$ & $\begin{array}{l}\text { Unklar } \\
\text { (fehlende Daten) }\end{array}$ \\
\hline Tigecyclin & $\begin{array}{l}\text { Einzelfälle; meist unter } \\
\text { Therapie }\end{array}$ & $\begin{array}{l}\text { Unklar } \\
\text { (fehlende Daten) }\end{array}$ & $\begin{array}{l}\text { Unklar (fehlende } \\
\text { Daten) }\end{array}$ & $\begin{array}{l}\text { Unklar } \\
\text { (fehlende Daten) }\end{array}$ & $\begin{array}{l}\text { Unklar } \\
\text { (fehlende Daten) }\end{array}$ \\
\hline Daptomycin & $\begin{array}{l}\text { Kein horizontaler Gen- } \\
\text { transfer bekannt }\end{array}$ & $\begin{array}{l}\text { Unklar } \\
\text { (fehlende Daten) }\end{array}$ & $\begin{array}{l}\text { Assoziation mit Einsatz } \\
\text { von Daptomycin }\end{array}$ & $\begin{array}{l}\text { Unklar } \\
\text { (fehlende Daten) }\end{array}$ & $\begin{array}{l}\text { Unklar } \\
\text { (fehlende Daten) }\end{array}$ \\
\hline
\end{tabular}

enthaltes insgesamt bzw. nach dem Erstnachweis des Linezolid-resistenten Erregers war in keiner der Studien signifikant erhöht.

Kainer et al. publizierten eine FallKontroll-Studie im Rahmen eines Ausbruchs mit Linezolid-resistenten Enterokokken [269]. Bei einer sehr geringen Fallzahl ( $n=15$, davon sieben LVRE) waren sowohl die Sterblichkeit $(\mathrm{OR}=9,3)$ als auch die stationäre Verweildauer für die Fallpatienten signifikant erhöht.

\subsubsection{Outcome bei Infektionen mit Tigecyclin-resistenten Enterokokken}

Aufgrund der derzeitigen Datenlage ist eine Einordnung der klinischen Relevanz der Tigecyclin-Resistenz bei Enterokokken nicht möglich.

Über den klinischen Verlauf bei Patienten mit Tigecyclin-resistenten Enterokokken liegen bisher kaum Informationen vor. Werner et al. beschrieben einen fatalen Ausgang bei einer 65-jährigen abdominalchirurgischen Patientin [107]; ein 65-jähriger, ebenfalls chirurgischer Patient konnte erfolgreich mit Piperacillin/ Tazobactam therapiert werden [270].

\subsubsection{Outcome bei Infektionen mit Daptomycin-resistenten Enterokokken}

Infektionen mit Daptomycin-resistenten Enterokokken wurden bisher über- wiegend bei Patienten, die Hochrisikokollektiven angehören, nachgewiesen. Die Sterblichkeit in diesen wenigen Fällen war hoch, kann jedoch bei sehr niedrigen Fallzahlen nicht abschließend eingeordnet werden.

Die Daptomycin-Resistenz bei Enterokokken ist nach wie vor niedrig $(<0,1 \%)$, dies erklärt möglicherweise das Fehlen vergleichender Studien. In einer Übersichtsarbeit von 2011 stellten die Autoren 150 Fälle mit Infektionen durch Daptomycin-resistente Enterokokken zusammen [271]. Angaben zum Outcome fanden sich jedoch nur in sieben Fällen, bei denen es zum Therapieversagen unter Daptomycin kam [272-278]. Zwei der Patienten mit Vancomycin- und Daptomycin- resistentem $E$. faecalis verstarben trotz eingeleiteter Folgetherapie mit Linezolid [272, 276].

In den Folgejahren wurden einige wenige kleine Fallserien mit Einschluss von 8-18 Patienten überwiegend aus Risikokollektiven (Stammzelltransplantierte, Hämatologisch-onkologische Patienten, Lebertransplantierte) publiziert [279282]. Die Sterblichkeit lag in diesen Fällen zwischen $21 \%$ [282] und $44 \%$ [280].

Eine Fall-Kontroll-Studie zum Vergleich des Outcomes zwischen 20 Patienten mit Sepsis durch Daptomycin-resistente VRE (Fälle) und 40 Patienten mit Sepsis durch Daptomycin-sensible VRE (Kontrollen) zeigte eine erhöhte Kompli- kationsrate bei Fällen, aber keinen Unterschied in der Gesamtsterblichkeit [283].

\subsection{Zusammenfassung der Bedeutung von Enterokokken mit speziellen Resistenzen}

Enterokokken gehören zu den am häufigsten nachgewiesenen Erregern bei nosokomialen Wund- und Harnwegsinfektionen und der katheterassoziierten Sepsis, wobei der Anteil von E. faecium stark zugenommen hat. Durch eingeschränkte therapeutische Möglichkeiten sind klinisch die Vancomycin-resistenten E. faecium von besonderer Bedeutung. Risikofaktoren für eine Infektion sind Komorbidität, vorausgegangene Antibiotikatherapie und die vorbestehende Kolonisation mit VRE. Je nach betroffener Patientenpopulation können bis zu 20 \% der kolonisierten Patienten eine Infektion erleiden. Am häufigsten treten schwerwiegende Infektionen in der Hämatologie-Onkologie, bei lebertransplantierten Patienten und auf Intensivstationen auf.

Die verfügbaren Daten deuten auf eine Übersterblichkeit bei VRE-infizierten Patienten hin, auch wenn die Qualität in Hinblick auf die vorliegenden Studien aufgrund der mitunter fehlenden Berücksichtigung von Störfaktoren limitiert ist. Es gibt Daten, die zeigen, dass sich das Outcome von Infektionen bei unterschiedlichen Enterokokken-Spezies un- 
terscheidet, wobei E. faecium-Infektionen ein schlechteres Outcome haben. Inwieweit die erworbene Vancomycin-Resistenz innerhalb einer Enterokokken-Spezies das Outcome beeinflusst, ist nicht abschließend geklärt, da ein Großteil der Studien nicht konsequent zwischen verschiedenen Enterokokken-Spezies unterschieden hat.

Von klinischer Bedeutung sind die Vancomycin-Resistenzen vom VanA- und VanB-Typ. Hier sind bislang nicht genügend Daten vorhanden, um einen Unterschied in der klinischen Bedeutung dieser beiden Van-Typen abzuleiten (• Tab. 4).

Obgleich also noch Fragen bezüglich der klinischen Bedeutung von VRE offen sind, müssen im Zweifelsfall zusätzliche Hygienemaßnahmen in Risikokollektiven umgesetzt werden, um die Patienten vor Infektionen zu schützen. Außerdem erhöht die empirische und gezielte Therapie von VRE den Einsatz von Reserveantibiotika und damit auch den Selektionsdruck auf Linezolid- oder Daptomycin-resistente grampositive Erreger, was es zu verhindern gilt.

Die gesichteten Publikationen weisen bisher nicht eindeutig darauf hin, dass die Linezolid-Resistenz allein oder die kombinierte Resistenz gegenüber Linezolid und Vancomycin eine erhöhte Morbidität oder Mortalität gegenüber der jeweils Linezolid-empfindlichen Variante verursacht. Andererseits sind bisher erst wenige Daten vorhanden. Da Linezolid derzeit das einzige zugelassene und als wirksam betrachtete Reserveantibiotikum für antibiotisch-therapiebedürftige Infektionen durch VRE ist, muss einer Verbreitung der Linezolid-Resistenz frühzeitig entgegengewirkt werden (Schutz der Sub$\operatorname{stanz})$.

Die Bedeutung der Resistenz gegenüber anderen Reserveantibiotika wie Tigecyclin oder Daptomycin kann noch nicht vollständig eingeschätzt werden, zumal der Stellenwert dieser Antibiotika für die Therapie schwerer Infektionen durch VRE ohnehin kritisch gesehen wird.

\section{Präventionsmaßnahmen}

Maßnahmen zur Prävention von VRE-
Infektionen oder -Kolonisationen wur-
den praktisch immer in Bündeln unter-
sucht. Die Studienqualität weist dabei hinsichtlich Design, Setting, Outcome und Studienziel erhebliche Heterogenität auf. In keiner Untersuchung konnte eine erfolgreiche Reduktion von VREInfektionen oder-Kolonisationen durch eine einzelne Maßnahme gezeigt werden. Erfolgreiche Bündel bestanden in der Regel aus mehr als zwei verschiedenen Maßnahmenpaketen.

Studien zur Prävention von VRE-Kolonisationen oder -Infektionen wurden von den Mitgliedern der Arbeitsgruppe durch Recherche in Medline identifiziert und hinsichtlich ihrer Relevanz bewertet.

Maßnahmen zur Prävention von Kolonisation und Infektion mit antibiotikaresistenten Enterokokken sind in nahezu allen publizierten Berichten als Bündel von Maßnahmen durchgeführt worden. Damit kann der Beitrag der Einzelmaßnahme zum Gesamterfolg nicht sicher angegeben werden.

Bestandteile der verschiedenen Maßnahmenbündel waren: Aktives oder passives Screening, Unterbringung der Patienten in Einzelzimmern, erweiterte Barrieremaßnahmen (Kittel und Handschuhe), verbesserte Händehygiene, intensivierte Aufbereitung der Patientenumgebung, antiseptisches Waschen der Patienten und Antibiotic Stewardship (ABS)-Programme.

Hinsichtlich des Studien-Designs, des Settings, der VRE-Raten und vor allem der Qualität der Studien gibt es erhebliche Unterschiede, so dass zusammenfassende Analysen nicht möglich sind.

Ein Großteil der Studien wurde als Vorher/Nachher-Vergleich bei Einführung der Intervention in einem oder mehreren Schritten durchgeführt. Nur in wenigen Fällen wurden zeitgleich Kontrollgruppen mitgeführt. Es wurden zum Teil alle Patienten des Krankenhauses und zum Teil nur die Patienten bestimmter Risikostationen betrachtet. Als Outcome gemessen wurden Raten der Kolonisation oder Neukolonisation, alle oder bestimmte Infektionen, oder der Anteil resistenter Isolate an allen identifizierten Isolaten.

Hinsichtlich des Zieles bzw. des gefundenen Ergebnisses unterschieden sich die Studien in solche, in denen das Ergebnis eine erfolgreiche Reduktion von VRE-Kolonisationen bzw. -Infektionen war, Studien ohne Erfolg der Maßnahmen, Studien, in denen Maßnahmen ersetzt wurden, ohne dass es zu einer Zunahme von Infektionen oder Kolonisationen kam, und Studien, in denen auf Maßnahmen verzichtet wurde, ohne dass es zu Veränderungen der Infektionsrate kam.

In der Anlage werden publizierte Studien tabellarisch zusammengestellt, um einen Überblick über gewählte Bündel und deren Ergebnisse zu geben. Es ist zu berücksichtigen, dass vielfach nur unzureichend Angaben über alle Rahmenbedingungen der Untersuchungen gemacht wurden. Am häufigsten wurden Studien mit einem positiven Ausgang, d.h. einer Reduktion von Kolonisations- oder Infektionsraten publiziert (- Tab. 5 in der Anlage). In den meisten der untersuchten Bündel sind eine Reihe von Maßnahmenpaketen (zum Teil vier und mehr) umgesetzt worden.

Weniger häufig wurden Untersuchungen publiziert, in denen es unter der Intervention nicht zu einer Reduktion der VRE-Infektionen oder -Kolonisationen kam, obgleich dies das Ziel der Intervention war (• Tab. 6 in der Anlage).

In Untersuchungen, in denen geprüft wurde, ob Maßnahmen ersetzt werden können, ohne dass es zu Veränderungen kommt, wurden zunächst die Gleichwertigkeit von gezieltem Tragen von Handschuhen und Kitteln gegenüber dem universellen Gebrauch von Handschuhen untersucht. In jüngerer Zeit wurde überwiegend untersucht, ob das generelle antiseptische Waschen aller Patienten gleichwertig zu Isolierungsmaßnahmen ist ( $\bullet$ Tab. 7 in der Anlage). In neueren Studien wurde untersucht, ob der ersatzlose Verzicht auf bestimmte Maßnahmen mit einer Zunahme der VRE-Infektionen oder -Kolonisationen einhergeht (- Tab. 8 in der Anlage). Für diese Untersuchungen ist kritisch anzumerken, dass in keinem Fall vorab eine statistische Bewertung vorgenommen wurde, wie groß die Untersuchungsgruppe sein müsste, um mit Sicherheit ausschließen zu können, dass es keine Zunahme gibt.

Im Folgenden werden die jeweils betrachteten Einzelmaßnahmen bzw. Maßnahmenpakete anhand von Untersuchungen bewertet, bei denen die jeweilige Maßnahme ein relevanter Bestandteil des Bündels (Intervention) war. 


\subsection{Screening}

Unter einem mikrobiologischen Screening im engeren Sinne wird die Untersuchung von nicht-invasiv entnommenem Material (hier meist Stuhl oder tiefe Rektalabstriche) von asymptomatischen $\mathrm{Pa}$ tienten verstanden.

Die Zielstellung von Screeninguntersuchungen auf MRE kann darin bestehen,

- durch geeignete Maßnahmen die

Weiterverbreitung der MRE im Krankenhaus zu verringern,

- im Falle von akuten Infektionen (z. B. Sepsis) einen Hinweis für die empirische Therapie des gescreenten Patienten zu erhalten,

- das Bestehen einer Kolonisation bereits bei Aufnahme aus forensischen Gründen zu dokumentieren und/oder die Rückverlegung des Patienten abzusichern.

Die folgenden Abschnitte adressieren nur das erstgenannte Ziel, d.h. den Benefit eines Screenings für die anderen Patienten und das Krankenhaus, nicht die klinischen Vorteile für den gescreenten $\mathrm{Pa}$ tienten, die sich aus der Kenntnis des VRE-Status für individuelle Therapieentscheidungen oder Einschätzungen zur individuellen Prognose ergeben können.

\subsubsection{Effektivität von Screening} Unter Berücksichtigung der aktuell publizierten Daten ist die Evidenz für systematisches VRE-Screening aller Patienten inkonsistent.

Dennoch waren ein aktives Screening (Untersuchung von für die VRE-Diagnostik gezielt entnommenen Proben) zumindestens von Risikopatienten oder passives Screening (Untersuchung von Proben, die den Patienten ohnehin aus klinischer Indikation entnommen wurden) wesentlicher Bestandteil einer Reihe von erfolgreichen Bündeln zur Prävention von VRE-Kolonisationen und -Infektionen.

Unter aktivem Screening wird mindestens die Untersuchung aller Patienten oder bestimmter Patientengruppen bei Aufnahme in eine medizinische Einrichtung verstanden. Manchmal werden auch während des Aufenthaltes und/oder bei Entlassung entnommene Surveillance-Pro- ben als Bestandteil des aktiven Screenings angesehen. Im Gegensatz dazu werden bei passivem Screening nur solche Proben auf VRE untersucht, die den Patienten ohnehin aus klinischer Indikation entnommen wurden (auch wenn nicht notwendig vom behandelnden Arzt die Untersuchung auf VRE explizit angefordert war, sondern beispielsweise auf $C$. difficile).

Im Rahmen von VRE-Ausbrüchen wird aktives Screening bei Aufnahme (und teilweise im Verlauf) oft als Element der erfolgreichen Ausbruchkontrolle benannt. Von besonderem Interesse im Rahmen dieses Dokuments ist jedoch die Bewertung von routinemäßig durchzuführenden Screening- und Präventionsmaßnahmen in einem klinischen Setting mit regelmäßigem Neueintrag von VRE (endemisches Setting).

Zwei kontrollierte Interventionsstudien haben die Auswirkung von VREScreening und daraus abgeleiteten Hygienemaßnahmen auf die Inzidenz von im Krankenhaus erworbenen VRE untersucht. Eine große Cluster-randomisierte kontrollierte Studie wurde in den USA auf zehn Interventions- und acht Kontroll-Intensivstationen durchgeführt [9]. Auf allen Stationen sollte neben der Basishygiene eine Barrierepflege (Kittel/ Handschuhe, patientenbezogene Medizinprodukte) bei bekannten VRE-Trägern durchgeführt werden. Dies betraf in beiden ITS-Gruppen etwa $4 \%$ der Patienten. Im Rahmen der Studie erfolgte ein Aufnahmescreening auf VRE (und parallel auf MRSA), womit zusätzlich etwa 1,5 \% der Patienten als VRE-positiv identifiziert wurden. Diese Ergebnisse waren nach zwei Tagen verfügbar und wurden auf den Interventionsstationen dem Personal mitgeteilt, womit neu diagnostizierte VRE-Träger der Barriereisolierung unterworfen werden konnten. Auf den Kontrollstationen wurden die Ergebnisse nicht mitgeteilt. Im Rahmen der Studie erfolgten außerdem auf allen Stationen wöchentliche Surveillancekulturen. Die Inzidenz neuer VRE-Fälle während des Stationsaufenthaltes war in beiden Gruppen nicht signifikant unterschiedlich, die Intervention „aktives VRE-Screening " brachte also keinen Vorteil. Als Kritik an dieser Studie wurde angeführt, dass die Compliance des medizinischen Perso- nals mit den Hygienemaßnahmen auf den Interventionsstationen nicht ausreichend gewesen wäre: frische Handschuhe wurden im Median bei $82 \%$ der Patientenkontakte benutzt, Kittel in $77 \%$ und Händedesinfektion nach Patientenkontakt in 69 \% der Fälle. Weiterhin eingeschränkt wird die Aussagekraft dieser Studie dadurch, dass auf die Barrieremaßnahmen direkt am Patienten fokussiert wurde und der Aspekt der Flächendesinfektion nicht diskutiert wurde. So bleibt unklar, ob und welche laufenden Desinfektionen oder Schlussdesinfektionen durchgeführt wurden. Dies muss bei einem Erreger mit hoher Persistenz in der Umwelt als deutliche Limitation angesehen werden.

Eine große europäische Cluster-randomisierte kontrollierte Studie (MOSAR WP3) auf 13 Intensivstationen evaluierte einen möglichen Zusatznutzen von MREScreening und Isolierung der als MRE-positiv identifizierten Patienten [285]. Dies erfolgte im Rahmen der Studie in einem kontrollierten Setting, in dem während einer vorhergehenden Studienphase eine deutliche Verbesserung der Händehygiene auf über $80 \%$ erreicht und eine allgemeine desinfizierende Körperwaschung aller Patienten mit Chlorhexidin eingeführt wurde. Die Kombination dieser Maßnahmen führte zu einer Reduktion nosokomialer, d.h. auf den Studien-ITS erworbener, VRE-Fälle. In einer weiteren Studienphase wurden Screeningproben bei ITS-Aufnahme entnommen und unter anderem auf VRE untersucht, wobei kulturelle Verfahren bzw. NAAT zum Einsatz kamen. In keiner dieser beiden Verfahren war eine weitere Reduktion nosokomial erworbener VRE-Fälle nachweisbar.

Mittels eines multimodalen VREKontrollprogramms (Isolierungsmaßnahmen mit Barrierepflege, räumliche Isolierung, intensivierte Flächendesinfektion mit verändertem Desinfektionsmittel zur Schlussdesinfektion und Kontrolle der Reinigungsqualität, generell verbesserte Basis- und insbesondere Händehygiene) in einem Krankenhaus in Singapur konnte die VRE-Inzidenz von 1,5/1000 Aufnahmen auf 0,5/1000 Aufnahmen reduziert werden [286]. Die Studie könnte jedoch für ein endemisches Auftreten nicht repräsentativ sein, da die Mehrzahl der identifizierten Fälle später als 48 Stun- 
den nach Aufnahme auftraten und es sich in $80 \%$ der Fälle genotypisch um denselben VRE-Klon handelte. Es hatte sich also möglicherweise um ein epidemisches VRE-Vorkommen im Sinne eines Ausbruches gehandelt [286].

In einem kanadischen Krankenhaus der Maximalversorgung wurden umfangreiche VRE-Kontrollmaßnahmen (aktives Screening aller aufgenommenen Patienten, räumliche Isolierung VRE-positiver Patienten mit Kittel-/Handschuhpflege, wöchentliche Surveillance-Kulturen aller Patienten auf Stationen mit mindestens einem VRE-Patienten sowie teilweise Einrichten einer VRE-Kohorte) reduziert auf risikobasiertes VRE-Screening bei Aufnahme (Aufnahme auf Risikostationen, z. B. Hämatologie-Onkologie, ITS, Neonatologie, oder bei Übernahme aus Einrichtungen mit hoher VRE-Prävalenz) sowie Verlegungen [287]. Nach Reduktion der VRE-Kontrollmaßnahmen kam es zu einem Anstieg der VRE-Bakteriämien von etwa 0-2 Fällen pro Quartal auf 0-6 Fälle pro Quartal sowie zu einer deutlich gestiegenen Zahl von VRE-Kolonisationen [287].

Fokussiert auf die Erwachsenen-Onkologie wurde in den USA ein multimodales VRE-Kontrollprogramm mit den wesentlichen Komponenten Händehygiene, Kohortierung von VRE-Patienten mit Kittel-/Handschuhpflege, räumlicher Isolierung und separatem Personal für VREpositive/negative Patienten eingeführt [288]. Bei der Zeitreihen-Analyse sank die Inzidenzdichte von VRE-Sepsisfällen von 2,1/1000 Patiententage (PT) auf 0,45/1000 PT; die Rate der VRE-Kolonisationen von 20,7/1000 PT auf 10,3/1000 PT [288].

Bei einem retrospektiven Vergleich zwischen zwei vergleichbaren Krankenhäusern der Maximalversorgung in der Region Chicago war in dem Krankenhaus mit aktivem VRE-Screening (bei Aufnahme auf Intensiv- und Transplantationsstationen) die Inzidenzdichte der VRE-Sepsis mit 8,2/100.000 PT knapp halb so hoch wie die Inzidenzdichte in dem Vergleichskrankenhaus ohne VRE-Screening und ohne Isolierung bekannter VRE-Träger (17/100.000 PT) [289]. Außerdem war in dem Krankenhaus ohne VRE-Screening/ -Isolierung bei den aus klinischen Proben isolierten VRE ein oligoklonales
Muster vorherrschend (vier Klone verantwortlich für $75 \%$ der VRE-Bakteriämien), während in dem Haus mit aktivem VRE-Screening und Isolierung nur 37 \% zu den Stämmen gehörten, die auch bei anderen Patienten nachweisbar waren und somit wahrscheinlich im Krankenhaus übertragen worden waren. Diese Studie ist jedoch wegen des retrospektiven Designs und fehlender Daten zu Basishygiene- und Isolierungsmaßnahmen nur von eingeschränkter Aussagekraft. Die Ergebnisse des in einem der teilnehmenden Krankenhäusern durchgeführten aktiven VRE-Screenings (Anteil tatsächlich gescreenter Patienten an der zu screenenden VRE-Risikopatientengruppe, VRE-Prävalenz bei Aufnahme in dieser Gruppe) wurden nicht dargestellt, was die Aussagekraft weiter einschränkt.

In einer ländlichen Region der USA traten bis 1996 keine VRE auf, nach ersten Fällen wurde 1997 eine regionale Kontrollstrategie unter Beteiligung der 32 regionalen Krankenhäuser etabliert [290]. $\mathrm{Zu}$ den Präventionselementen gehörten aktives VRE-Screening und Isolierung VRE-positiver Patienten. Es gelang, die während des Krankenhausaufenthaltes festgestellte Prävalenz in der Region von 2,2 \% (1997) auf 0,5 \% (1999) zu senken [290].

Die Prävalenz von VRE kann in Stuhlproben von Patienten mit C. difficile-Infektionen signifikant höher sein als in Vergleichsstuhlproben [291, 292]. Daher wurde die Untersuchung von Stuhlproben, die zur Untersuchung auf $C$. difficile eingesandt wurden auf das Vorhandensein von VRE als Screeninginstrument genutzt $[292,293]$.

In einer Zeitreihenuntersuchung, in der passives Screening von Stuhlproben, die zur Untersuchung auf $C$. difficile-Toxin in einem amerikanischen 1250-Betten-Krankenhaus eingesandt wurden, ausgesetzt und wieder eingeführt wurde, zeigte sich eine signifikante Zunahme der VRE-Infektionsrate in der Zeit ohne passives Screening. Nach Wiedereinführung des passiven Screenings ging die Infektionsrate wieder zurück. VRE-positive $\mathrm{Pa}$ tienten wurden in einem Einzelzimmer mit Kittel und Handschuhen gepflegt [294, 295].

\subsubsection{Screeningmethoden}

Die Sensitivität der Untersuchung von Rektalabstrichen für Screeningszwecke ist geringer als die von Stuhlproben, sie beträgt ca. 60 \% für Rektalabstriche im Vergleich zur Stuhlprobe. Durch wiederholte Untersuchungen kann die Sensitivität erhöht werden. Als Labormethoden stehen die Kultur auf selektiven Screeningmedien oder NAAT aus dem Abstrich zur Verfügung. Es ist zu berücksichtigen, dass ein Direktnachweis des vanB-Gens kulturell bestätigt werden muss.

Einen wesentlichen Faktor für den Erfolg eines aktiven Screeningprogrammes stellen mit hoher Wahrscheinlichkeit die eingesetzten Screeningmethoden dar, sofern man davon ausgeht, dass der Erfolg von der Sensitivität der Erkennung von VRE-Trägern abhängt. Die Prävalenz der Besiedelung mit VRE kann schwierig zu erkennen sein, solange die meisten Nachweise und Resistenztestungen im klinischen Alltag nur aus primär sterilen Materialien oder Urin erfolgen [296].

Untersuchungsmaterialien. Die diagnostische Genauigkeit (Sensitivität und Spezifität) der Rektalabstriche mit der Kulturmethode bei der Identifizierung von gastrointestinaler Kolonisation mit VRE ist nicht bekannt. Serielle quantitative Stuhl-, Haut- und Rektalabstriche wurden für Patienten mit VRE-Infektionen durchgeführt, um die falsch-negative Rate der Rektalabstriche und die Prävalenz der Hautkolonisation bei unterschiedlichen VRE-Stuhldichten $\mathrm{zu}$ beurteilen [297]. Dabei zeigte sich, dass die Positivrate von Rektalabstrichen von der Dichte der VRE im Stuhl abhängig war. Bei einer VRE-Dichte von $\geq 7,5 \log _{10}$ $\mathrm{KBE} / \mathrm{g}$ Stuhl lag die Sensitivität bei $100 \%$ und fiel auf $0 \%$ ab bei einer Dichte von $\leq 4,5 \log _{10} \mathrm{KBE} / \mathrm{g}$ Stuhl. Die VRE-Dichte war dabei unter Antibiotikatherapie des Patienten signifikant höher. Die mittlere Sensitivität von Rektalabstrichen lag bei $58 \%$ [297].

Ein weiterer Einflussfaktor für die Sensitivität der Untersuchung kann die Wahl der Abstrichtupfer sein, da unterschiedliche Tupfer unterschiedlich viele Bakterien aufnehmen bzw. wieder abgeben können [298]. 
In einer australischen Kohorte von nahezu 2000 Patienten waren vier Abstriche, an verschiedenen Tagen entnommen, notwendig um $>90 \%$ der VRE-Träger zu detektieren [299]. Bei der Suche nach Patienten, die durch Kontakt zu VRE-Patienten VRE-Träger geworden sind, konnte der höchste Anteil an positiven Patienten sieben Tage nach dem initialen Kontakt mit einem Indexpatienten gefunden werden [137]. Wurden bei Kontaktpatienten zwei Abstriche im Abstand von sieben Tagen entnommen, so konnte eine Sensitivität von $80 \%$ erreicht werden [136].

Stuhl- und Rektalabstrichproben weisen in der Praxis jeweils relative Vor- und Nachteile für die Erkennung von VRE-besiedelten Patienten auf [300]. Rektalabstriche können jederzeit entnommen werden und sind nützlich, wenn eine große Anzahl von Proben gesammelt werden muss. Andererseits können Stuhlproben leichter vom Patienten selbst gewonnen werden und stellen eine Alternative für bestimmte Situationen (z. B. Chemotherapie, Strahlentherapie, ethische Vorbehalte) dar, in denen Rektalabstriche kontraindiziert bzw. nicht erwünscht sind.

Gelegentlich konnten antibiotikaresistente Enterokokken in der Nase, im Rachen oder in der Mundhöhle, aber nicht im gleichzeitig gewonnenem Rektal- oder Perinealabstrich nachgewiesen werden [301]. Der generelle Nutzen des Nasenbzw. Rachenabstrichs für Screeningzwecke erscheint jedoch fraglich.

Labormethodik. Der Nachweis von VRE beruht traditionell auf der Kultur, die 2472, z. T. auch 96 Stunden zur Isolierung, Identifikation und Empfindlichkeitstestung benötigt.

Wird gezielt nach Enterokokken aus Rektalabstrichen oder Stuhlproben gesucht, werden zur Unterdrückung der schneller wachsenden und dominierenden Begleitflora Selektivmedien eingesetzt, welche die natürliche Resistenz der Enterokokken gegen Galle und Äsculin ausnutzen. Zur Selektion von VRE werden den Screeningmedien zudem unterschiedliche Konzentrationen von Vancomycin zugesetzt [302]. Zu berücksichtigen ist, dass die zugesetzte Vancomycin-Konzentration die Sensitivität und Spezifität der Medien beeinflussen kann.
Durch die Verwendung von selektiven chromogenen Nährmedien kann eine schnellere Screeningdiagnostik mit einer erhöhten Sensitivität für den Nachweis von VRE aus Stuhlproben und Rektalabstrichen erzielt werden. Mehrere Formulierungen von kommerziell hergestelltem chromogenen Agar zum Nachweis von VRE sind derzeit im Handel. Alle enthalten Vancomycin zur Selektion von VRE. In verschiedenen Studien wurden herkömmliche Galle-Äsculin-Salz-Nährböden mit Vancomycin gegen verschiedene chromogene Medien, sowie chromogene Medien untereinander verglichen [303305]. Chromogene Medien zeigen im Allgemeinen eine höhere Sensitivität als herkömmliche Galle-Äsculin-Salz-Nährböden ( $\geq 90 \%$ gegenüber $85 \%$ ), weisen jedoch zum Teil geringfügig geringere Spezifitäten auf (98\% gegenüber $100 \%$ ) [87, 305].

Es stehen ebenfalls NAAT für den Nachweis von VRE zur Verfügung, welche unterschiedlich komplizierte Extraktionsund Nachweisverfahren und zum Teil auch einen Kulturschritt erfordern. Einzelne Testverfahren benötigen eine Anreicherungsbouillon bzw. über feste Medien gewonnene Isolate [302]. Dabei erhöht eine Anreicherungsbouillon die Sensitivität, führt aber zur Verzögerung der Diagnostik [306]. Einige molekulargenetische Testverfahren besitzen nur für bestimmte Materialien (z. B. Perianal-/Rektalabstriche) eine Zulassung. Die Sensitivität der NAAT wurde mit 61-100\% angegeben, die Spezifität mit 69,3-99,6 \% [87].

Ein Nachteil der NAAT ist die geringe Spezifität für den Nachweis des vanB-Gens in Enterokokken, die zu verhältnismäßig häufigen falsch-positiven Testergebnissen führt. Diese Nachweise müssen kulturell bestätigt werden [307, 308]. So liegt die Spezifität für den Nachweis des vanA-Gens bei $99 \%$, während die des vanB-Gens bei $87 \%$ liegt [307]. Je nach Prävalenz kann damit der positive Vorhersagewert der NAAT bei nur $30 \%$ liegen [307, 308]. Ggf. kann der hohe negative Vorhersagewert in Populationen mit hohem Kolonisationsrisiko zum Ausschluss der VRE-Besiedelung genutzt werden.

Ein weiteres Problem im Zusammenhang mit der Verwendung von NAAT ist die fehlende Möglichkeit, die Isolate für ggf. notwendige epidemiologische Untersuchungen bei Häufungen zu sammeln und teilweise auch die mangelnde Differenzierung zwischen E. faecium und $E$. faecalis [309]. Eine Zeitersparnis bei direkt aus einer Anreicherungsbouillon durchgeführten vanA-/vanB-Nachweis mittels NAAT konnte gezeigt werden [310].

Der Vorteil bei Einsatz molekulargenetischer Testverfahren ist vor allem die niedrigere Turnaround-Zeit gegenüber den traditionellen Kulturmethoden. Andererseits können die Kosten signifikant höher sein als die der Kultur. Daten zur epidemiologischen oder klinischen Relevanz einer Zeitersparnis legen nicht vor.

\subsection{Isolierung}

Durch Unterbringung in Einzelzimmern in Verbindung mit Barrieremaßnahmen (Kontaktisolierung) auf der Basis von Ergebnissen von Screeninguntersuchungen wurde in vielen, aber nicht allen Studien eine Reduktion von VRE-Infektionen und horizontaler Transmission erreicht.

Die alleinige Durchführung von Barrieremaßnahmen (Handschuhe und Schutzkittel) reicht zur Kontrolle der VRE-Transmission nicht aus.

Ein Verzicht auf Screening und auf Kontaktisolation von VRE-positiven Patienten hatte in einigen Studien keinen Einfluss auf die nosokomiale Infektionsrate, Angaben zu nosokomialen Transmissionen wurden nicht gemacht.

Eine Schwierigkeit bei der Interpretation der Untersuchungen zum Stellenwert der Isolierung stellt die wenig einheitliche Verwendung der Begriffe „barrier precaution" und "contact isolation“ in den Publikationen dar. Oft ist nicht klar zu erkennen, wann die Maßnahme die Unterbringung in einem Einzelzimmer voraussetzte und wann die Maßnahmen zusätzlich zur Unterbringung im Einzelzimmer das Tragen von Handschuhen und Kittel für jeden Kontakt einschlossen. Im Folgenden wird der Begriff Barrieremaßnahmen für die erweiterte Verwendung von Kitteln und Handschuhen bei allen Patientenkontakten und ggf. bei Zutritt zum Zimmer verwendet. Kontaktisolierung bezieht neben den Barrie- 
remaßnahmen die Unterbringung im Einzelzimmer mit ein.

In nahezu allen Untersuchungen zum Stellenwert der Isolierung zur Prävention der Weiterverbreitung von VRE waren die Isolierungsmaßnahmen Teil eines Interventionsbündels und wurden zumeist mit intensivierten Screeningmaßnahmen verbunden. Ganz überwiegend wurden Ausbruchsszenarien beschrieben, bei denen für die Intervention keine geeignete Kontrollgruppe zur Verfügung stand.

Exemplarisch seien hier drei Interventionsstudien zur Kontrolle gehäuften VRE-Auftretens erwähnt. Ostrowsky et al. zeigten 2001 in einer dreijährigen Interventionsstudie in 30 US-amerikanischen Institutionen einen Rückgang der VREPatienten durch Einzelzimmer- bzw. Kohortenisolierung und Barrieremaßnahmen [290]. Lucet et al. beschrieben die erfolgreiche Beendigung eines VRE-Ausbruchs mit 37 Patienten durch Kohortierung der Patienten auf einer Isolierstation und extensive Screeningmaßnahmen [311]. Fournier et al. verfolgten verschiedene Strategien über einen Zeitraum von sieben Jahren, in dem insgesamt 45 durch E. faecium verursachte Ausbrüche in einem Krankenhausverbund auftraten [217]. Während Barrieremaßnahmen ohne räumlich getrennte Unterbringung keinen Erfolg hatten, konnte durch Implementierung eines Maßnahmenbündels mit Unterbringung der Patienten in drei räumlich und organisatorisch getrennten Bereichen (VRE-Patienten/VRE-Kontakte/VRE-freie Patienten) ein signifikanter Rückgang der VRE-Fälle erreicht werden [217].

Price et al. verglichen zwei US-Krankenhäuser hinsichtlich des routinemäBigen Screenings auf VRE [289]. Eine Klinik mit aktivem Screening und Kontaktisolierung positiver Patienten sowie präemptiver Isolierung von Risikopatienten hatte signifikant weniger VREBakteriämien (8,2/100.000 Patiententage vs. 17,1/100.000 Patiententage) und eine größere Vielfalt der typisierten VREStämme im Vergleich zu einer Klinik ohne Screening und Isolierung. Die Autoren folgerten, dass aktive Surveillance und Kontaktisolation sowohl die Rate der VRE-Bakteriämien als auch die horizontale VRE-Transmission reduziert.
Calfee et al. zeigten, dass die Einführung von Kontaktisolierung kombiniert mit Surveillancekulturen zu einer Senkung der Inzidenzrate der VRE-Besiedelung von einem Peak bei 2,07 \% auf 1,25\% führte [216]. Wang et al. stellten in einer deskriptiven Arbeit einen Anstieg der VRE-Infektionsrate von 0,03-0,09/1000 Entlassungen in der Interventionsphase mit Kontaktisolierung auf 0,2/1000 Entlassungen fest, nachdem die Interventionen aufgegeben worden waren [312].

Bearman et al. untersuchten generelles Tragen von Schutzhandschuhen im Vergleich zur Kontaktisolation auf einer internistischen Intensivstation [313]. Die Autoren sahen signifikant niedrigere ( $p$ jeweils $<0,001)$ nosokomiale Infektionsraten pro 1000 Device-Tagen für Bakteriämien, Harnwegsinfekte und Beatmungspneumonien, jedoch keine Unterschiede bezüglich der Akquisition von MRSA und VRE. Drei Jahre später publizierten Bearman et al. die Ergebnisse einer vergleichbaren Intervention, diesmal auf einer chirurgischen Intensivstation, ebenfalls ohne signifikante Unterschiede in der Prävalenz von MRSA und VRE [314].

Eine randomisierte Studie von Huskins et al. mit 18 teilnehmenden Intensivstationen postulierte keinen Einfluss von Surveillancekulturen und Isolationsmaßnahmen auf die VRE-Transmissionsrate [9]. Leider erfolgte die Kontaktisolierung VRE-positiver Patienten erst nach Vorlage des Ergebnisses des Aufnahmescreenings ( $\geq 48$ Stunden), so dass bei der Bewertung ein erheblicher Bias durch die Möglichkeit der nosokomialen Übertragung in diesem Zeitraum berücksichtigt werden muss.

Harris et al. untersuchten in einer randomisierten Studie mit 20 teilnehmenden Intensivstationen den Effekt des generellen Tragens von Schutzkleidung (Kittel und Handschuhe) bei allen Patientenkontakten (Interventionsgruppe) im Vergleich zum gezielten Tragen nur bei $\mathrm{Pa}-$ tienten mit bekannter MRE-Besiedelung (Kontrollgruppe) [315]. In der Interventionsgruppe kam es zu einem Rückgang der MRSA-Neubesiedelungen, nicht jedoch zu einem Rückgang der VRE-Akquisition. Dies könnte als Hinweis gewertet werden, dass Barrieremaßnahmen allein keinen Effekt auf die Weiterverbreitung von VRE haben.
Mehrere Reviews versuchten, übergreifende Aussagen zum Stellenwert der Kontaktisolierung von Patienten mit multiresistenten Erregern zu treffen [316-318]. Aboelela et al. bewerteten in ihrem Review Publikationen zum Thema und wiesen auf die Inhomogenität der zumeist monozentrischen, retrospektiven Studien hin, die vor allem deskriptive oder „quasiexperimentelle" Designs aufwiesen [316]. Häufig gab es keine Angaben zur Compliance mit den Interventionsmaßnahmen. In keiner Studie wurde der Effekt einer einzelnen Interventionsmaßnahme wie der Kontaktisolierung unabhängig von begleitenden weiteren Maßnahmen geprüft. Ebenso fehlen häufig Angaben zur tatsächlichen (beobachteten) Compliance mit den Maßnahmen (z. B. Händedesinfektion).

In aktuellen Studien wurde geprüft, welche Konsequenzen der Verzicht auf aktives Screening und auf die generelle Isolierung VRE-positiver Patienten hat. Edmond et al. sahen keinen Einfluss auf die Rate der Device-assoziierten Infektionen nach Beendigung der Kontaktisolierung für MRSA- und VRE-Patienten, wobei die Rate der Isolierungstage nur um $45 \%$ reduziert wurde [319]. Patienten mit einem besonderen Streuungsrisiko (z.B. stark sezernierende Wunden) wurden weiter isoliert. Eine tägliche Chlorhexidin-Waschung aller Patienten wurde in beiden Perioden durchgeführt. Almyroudis et al. untersuchten hämatologisch-onkologische Patienten in einer Abteilung, in der alle Patienten in Einzelzimmern mit eigener Nasszelle untergebracht waren und eine tägliche Chlorhexidin-Waschung erhielten [320]. Die Intervention bestand ausschließlich in der Aufgabe des aktiven Screenings sowie dem Verzicht auf das generelle Tragen von Schutzkleidung. Hierunter stellten sie keinen Anstieg der VREBakteriämien fest [320]. In einer weiteren Studie an einem 800 Betten-Krankenhaus fanden die Autoren ebenfalls keine $\mathrm{Zu}$ nahme von VRE-Infektionen nach Beenden der Barrieremaßnahmen [321]. Chlorhexidin-Waschungen wurden beibehalten. Alle 140 Intensivbetten der Klinik waren Einzelzimmer und die Compliance mit der Händehygiene lag bei $78 \%$ [321]. Angaben über die Anzahl der nosokomialen Transmissionen/VRE-koloni- 
sierten Patienten wurden in den Arbeiten nicht gemacht. Die Untersuchungen waren bezüglich der Fähigkeit, einen Unterschied aufzuzeigen (statistische Power), zu klein.

Eine Meta-Analyse zu sechs Studien, in denen Isolierungsmaßnahmen zur Prävention von VRE-Infektionen aufgegeben wurden, und die die drei oben genannten Studien einschließt,ergab eine signifikante Reduktion von VRE-Infektionen nach Beenden der Isolierungsmaßnahmen [322]. Eine Analyse der stattdessen umgesetzten Maßnahmen wurde nicht vorgenommen. Auffällig ist jedoch, dass die Studien, die über $90 \%$ der Wichtung der Daten einbrachten, in einem Setting mit ausschließlich oder einem hohen Anteil an Einzelzimmern durchgeführt wurden [322].

\subsection{Maßnahmen der Basishygiene und Barrieremaßnahmen}

Die Einhaltung der Basishygiene ist die wichtigste Präventionsmaßnahme. Sie ist auch bei den bei VRE häufig auftretenden unerkannten Reservoiren in kolonisierten aber unerkannten Patienten oder in der Umwelt wirksam.

In einigen, nicht immer kontrollierten, Studien konnte gezeigt werden, dass insbesondere die Händehygiene sowie in einigen Fällen das über die Basishygiene hinausgehende Tragen von Kitteln und Handschuhen (Barrieremaßnahmen) zur Prävention von VRE-Übertragungen beiträgt.

Händedesinfektion. Sowohl die Berührung des kolonisierten Patienten als auch der Kontakt mit Oberflächen in Räumen, die mit kolonisierten Patienten belegt sind, kann zur Kontamination der Hände führen [162]. Mathematische Modelle zur Übertragungswahrscheinlichkeit und zur Ausbreitung von VRE über die kontaminierten Hände des pflegerischen und ärztlichen Personals weisen darauf hin, dass multimodale Maßnahmen zur Verbesserung der Compliance mit der Hygiene eingesetzt werden müssen, um die gewünschten Ziele zu erreichen [323].

Venkatesh et al. erzielten durch ein elektronisches Alarmsystem eine Erhöhung der Compliance mit der Händehygiene auf $70 \%$, die gleichzeitig zu einer
Reduktion der Häufigkeit von VRE-Übertragungen führte, die allerdings nicht das statistische Signifikanzniveau erreichte [324]. Eine Meta-Analyse aus dem Jahr 2014 zieht die Schlussfolgerung, dass durch die Implementierung und Überprüfung der regelrechten Durchführung der Händedesinfektion eine 47-prozentige Reduzierung der Kontamination mit VRE erfolgte [318]. Andererseits fanden Jayaraman et al. auf Intensivstationen mit hoher Händehygiene-Compliance (>90 \%) keine Assoziation zwischen den Complianceraten und der Rate von MRE-Nachweisen inklusive VRE [325].

Einmalhandschuhe. Hayden et al. stellten fest, dass bei $52 \%$ der Mitarbeiter VRE auf den Händen nachgewiesen wurde, wenn sie nur den Patienten berührt hatten; dies war bei $70 \%$ der Mitarbeiter der Fall, wenn sie sowohl die Umgebung als auch den Patienten berührt hatten [162]. Das Risiko der Kontamination stieg wenn der Patient unter Durchfall litt und korrelierte mit der Anzahl der direkten Patientenkontakte, mit der Ausdehnung der Kolonisation sowie mit der Lokalistion der Infektion. In einer anderen Studie zeigte sich, dass VRE-Patientenisolate nach dem Ausziehen der Handschuhe auf den Händen der Mitarbeiter nachweisbar waren [326]. Hier reduzierten jedoch Handschuhe das Risiko, sich die Hände direkt mit VRE zu kontaminieren, um 71 \% [326].

Schutzkittel und Einwegschürze. Das Tragen von Einwegschutzkittel bzw. -schürzen konnte in zwei klinischen Studien mit Crossover-Design bei einem hohen VRE-Kolonisationsdruck das Risiko, intestinale VRE zu erwerben, signifikant reduzieren [327, 328]. Da andere mögliche Einflussfaktoren, wie die Compliance mit der Händehygiene nicht begleitend erfasst wurden, kann nicht ausgeschlossen werden, dass das Tragen der Kittel weitere nicht erfasste Effekte hatte. Dennoch weisen die Untersuchungen auf einen Wert von Kitteln bei der Isolierung von VRE-Patienten hin, sei es durch direkte Reduktion der Übertragung oder durch Verbesserung der Mitarbeit bei weiteren Hygienemaßnahmen.

In einer kontrollierten, randomisierten Studie zum Vergleich zwischen uni- versellem Tragen von Kitteln und Handschuhen bei jedem Patientenkontakt und jedem Zugang in ein Zimmer, und dem gezieltem Tragen von Kitteln und Handschuhen für Kontakte zu besiedelten $\mathrm{Pa}$ tienten, fanden sich keine Unterschiede in der Übertragungsrate zwischen beiden Studiengruppen [315].

\subsection{Schulung des Personals}

Gesonderte Schulungsprogramme für das Personal wurden nur in wenigen Präventionsbündeln dargestellt. Dennoch ist die Schulung des Personals eine Voraussetzung, Bündelmaßnahmen erfolgreich umzusetzen.

Perugini et al. führten im Rahmen eines prolongierten VRE-Ausbruchs ein Präventionsbündel ein, zu dem eine gezielte Schulung des Personals und Fragebogenerhebungen zählten [329]. Obgleich dies nicht zu einer Verbesserung der beobachteten Compliance mit Hygienemaßnahmen führte, wurde eine Reduktion der VRE-Infektionsraten und der Umgebungskontamination mit VRE beobachtet. Wiederum in einer Ausbruchsituation wurde mit Hilfe von Vorträgen und über das Intranet verbreitetem Informationsmaterial als Teil eines größeren Bündels mit weiteren Bestandteilen die Weiterverbreitung von VRE unterbunden [135].

In einer Veröffentlichung von Derde et al. wurde die Schulung zwar nicht als eigene Interventionsstrategie in der endemischen Situation beschrieben, jedoch in der Umsetzung von Maßnahmen, z. B. Verbesserung der Händehygiene vermerkt [285].

\subsection{Einbeziehung von Patienten}

Patienten wurden erfolgreich in Maßnahmenbündel zur Reduktion von VRE einbezogen, wobei der Fokus hier auf der Schulung zur Händehygiene lag.

Im Rahmen einer Interventionsstudie zur Reduktion der VRE-Inzidenz wurde ein Maßnahmenbündel implementiert, welches schwerpunktmäßig das Händewaschen vor der Einnahme von Mahlzeiten und oralen Medikamenten beinhaltete [330].

Die Einbeziehung von Patienten in die Umsetzung von Hygienemaßnah- 
men erfolgte auch in anderen Bündeln. So wurden Patienten geschult, nach der Benutzung der Toilette die Hände zu desinfizieren [331]. In beiden Studien konnte die Zahl neuer VRE-kolonisierter Patienten gesenkt werden.

\subsection{Reinigungs- und \\ Desinfektionsprogramme}

Intensivierte Programme zur Reinigung und Desinfektion der Patientenumgebung haben im Rahmen von Präventionsbündeln zur Reduktion der Inzidenz der Kolonisation geführt. In einer Untersuchung war ein Bündel aus intensivierter Umgebungsdekontamination und ABS dem aktiven Screenen und Isolieren in Niedrigrisikobereichen gleichwertig.

Verschiedene Untersuchungen weisen auf eine Rolle der Umgebungskontamination für die Transmission von VRE hin $[154,155,332]$. Dabei kann die Umgebungskontamination bei kolonisierten $\mathrm{Pa}$ tienten größer sein als bei infizierten $\mathrm{Pa}$ tienten [333]. Insbesondere bei Patienten mit Stuhlinkontinenz kann eine erhebliche Kontamination der Umgebung durch Enterokokken auftreten.

Wurden Oberflächen und Geräte nach Patientenkontakt bzw. Verwendung am Patienten desinfiziert, so kam es nur in Einzelfällen zu einer Übertragung auf Patienten, die die Zimmer nachfolgend belegten [334]. Daher wurde der Bedeutung der Reinigung und vor allem der Desinfektion der Patientenumgebung erhöhte Aufmerksamkeit gewidmet.

Im Ergebnis eines systematischen Reviews wurden unter anderem Studien bewertet, die den Einsatz von Wasserstoffperoxid $\left(\mathrm{H}_{2} \mathrm{O}_{2}\right)$-Verneblung zur Prävention von VRE-Übertragungen untersuchten [335]. Durch Reinigung mit nachfolgender Verneblung von $\mathrm{H}_{2} \mathrm{O}_{2}$ konnte in zwei Fällen ein Ausbruch beherrscht werden [335]. Später zeigten Passaretti et al., dass durch die Verneblung von $\mathrm{H}_{2} \mathrm{O}_{2}$ die Umgebungskontamination nach Patientenentlassung und die Akquisition von VRE signifikant herabgesetzt werden konnte [336]. Horn et al. stellten fest, dass durch den kombinierten Einsatz von $\mathrm{H}_{2} \mathrm{O}_{2}$ zur Schlussdesinfektion und Verbesserung der Händehygiene die VRERate gesenkt werden kann [337]. Die Häufigkeit von VRE war jedoch insgesamt gering und weitere Daten zum Umgang mit besiedelten Patienten werden nicht angegeben. $\mathrm{Zu}$ berücksichtigen ist, dass eine Raumdesinfektion durch Vernebeln mit Peroxiden nur als Ergänzung zur regulären Flächendesinfektion mittels Wischverfahren sinnvoll ist. Die Limitationen des Verfahrens sind zu berücksichtigen und die reproduzierbare Wirksamkeit der Maßnahme im Rahmen einer Validierung vor Ort zu prüfen [338, 339], was in den zitierten Studien nicht beschrieben wurde. Daneben ist der Aspekt des Mitarbeiter- und Patientenschutzes zu beachten, da zum Teil sehr hohe (toxische) Peroxidkonzentrationen in der Raumluft eingesetzt werden. Dadurch können die Räume ggf. erst nach längeren Lüftungsintervallen wieder genutzt werden [340, 341].

Durch den Einsatz eines Desinfektionsprogramms mit chlorbasierten Produkten (inklusive Schulung der Mitarbeiter und Einstellung von Supervisoren) mit gleichzeitig verstärktem Einsatz alkoholischer Händedesinfektionsmittel wurden in einem australischen Krankenhaus die Kontamination der Umgebung sowie die Inzidenz der Kolonisation und der VREBakteriämien signifikant reduziert [342]. Bryce et al. stellten in einem kanadischen Krankenhaus das VRE-Präventionsprogramm von krankenhausweitem aktivem Screening mit Kontaktisolierung der positiven Patienten auf ein risikobasiertes System mit Screening und Isolierung nur in Risikobereichen und einem ABS-Programm sowie einem verbesserten Dekontaminationssystem für Umgebung und Medizinprodukte um [343]. Darunter war keine Veränderung der VRE-Infektionsraten nachweisbar [343]. Bestandteile des Dekontaminationssystems waren Reorganisation des Lagersystems, Neuzuweisung der Verantwortlichkeiten und Kennzeichnung aufbereiteter Gegenstände sowie Benennung eines Programm-Managers und regelmäßige Kontrollen.

Weitere Interventionsmöglichkeiten beinhalten die Vermeidung der Kontamination mit der Stuhlflora. So wurde aufgrund steigender VRE-Prävalenz in Bereichen mit erhöhtem Risiko durch den Ersatz des Rektal- durch ein Ohrthermometer innerhalb von neun Monaten ein Rückgang der nosokomialen VRE-Infektionen um $48 \%$ erreicht [344].

\subsection{Antiseptisches Waschen der Patienten}

Antiseptisches Waschen führt auf Intensivstationen zur Reduktion der VRETransmission und VRE-Infektionen, vor allem der ZVK-assoziierten VRE-Sepsis. Ein gleichartiger Effekt konnte für Normalstationen nicht gezeigt werden.

In verschiedenen Studien wurde der Effekt einer täglichen antiseptischen Waschung auf die Transmissionsrate bzw. die Infektionsrate von VRE untersucht [285, 345-347]. In einer Meta-Analyse von zwölf Studien fanden die Autoren eine signifikante Reduktion sowohl der VRE-Infektionsraten als auch der Transmissionsraten [348]. Die Untersuchungen wurden nahezu alle auf Intensivtherapiestationen durchgeführt. Die Intervention bestand aus Waschen der Patienten mit Chlorhexidin in der Konzentration 2-4\%. Eine Studie auf internistischen Normalstationen nutzte in einem quasi-experimentellen Design ein zusammengefasstes Outcome von MRSA- oder VRE-Infektionen und zeigte eine Reduktion dieser zusammengefassten Größe [346]. Dabei veränderten sich neben der eigentlichen Intervention auch die Compliance mit der Händehygiene und den Barrieremaßnahmen. Aus dieser Untersuchung lässt sich herleiten, dass 770 Patienten mit Chlorhexidin gewaschen werden mussten, um eine Infektion $\mathrm{zu}$ verhindern (number needed to treat).

Dagegen fanden Dicks et al. in einer Multicenter-Studie an 33 Intensivstationen in insgesamt 17 Krankenhäusern keinen Effekt auf die VRE-Infektionsrate im Vergleich zwischen Stationen, die Chlorhexidinwaschungen vornahmen und Kontrollstationen [349]. Lediglich auf Interventions-ITS mit Waschungen konnte eine Reduktion der VRE-bedingten, ZVK-assoziierten VRE-Sepsis erzielt werden [349]. In einem anderen Ansatz wurden in einem Krankenhaus der Maximalversorgung und einem städtischen Krankenhaus das Waschen aller Patienten mit Chlorhexidin eingeführt und zeitgleich auf die Durchführung von Barrieremaßnahmen verzichtet [350]. Hierbei zeigte sich keine Veränderung der Rate an klinischen VRE-Nachweisen als Indikator für Infektionen. In beiden Häusern 
waren nahezu alle Betten in Einzelzimmern aufgestellt.

Bei einer häufigen Anwendung von Chlorhexidin sollte berücksichtigt werden, dass es zur Toleranz von E. faecium gegenüber der Substanz kommen kann [351], so dass andere Hautantiseptika von Interesse wären.

Nur in einer Studie wurde für die antiseptische Waschung nicht Chlorhexidin verwendet. Gastmeier et al. führten eine Interventionsstudie auf acht Intensivstationen durch und verwendeten Octenidin zur antiseptischen Waschung [347]. Gleichzeitig durfte auf Screening und Kontaktisolierung der Patienten verzichtet werden, wobei davon nicht alle Stationen Gebrauch machten. Ein Effekt auf nosokomialen VRE-Erwerb oder die Infektionsraten von VRE konnte hier nicht nachgewiesen werden [347].

\subsection{Maßnahmen der Eradikation}

Versuche einer Eradikation von VRE mit Antibiotika haben sich bisher als unwirksam erwiesen. Erste Untersuchungen zum Einsatz von Probiotika und zur FMT an kleinen Patientengruppen wurden durchgeführt, eine Evaluation in größeren Gruppen steht jedoch aus. Zusammenfassend sind die bisherigen Ergebnisse noch so vorläufig, dass der Einsatz außerhalb von klinischen Studien nur nach gründlicher Abwägung bei kritisch kranken Patienten erfolgen sollte.

Maßnahmen der Eradikation wurden bisher nur in kleinen Fallserien beschrieben. Hierzu kamen verschiedene Antibiotika und Probiotika zum Teil in Kombination zum Einsatz.

Dabei waren die Ergebnisse einer alleinigen Anwendung von Antibiotika unbefriedigend. Die Anwendung von Bacitracin führte zwar zu einer Clearance zwischen $43 \%$ und $100 \%$ [352-355], der Effekt hielt im 3-Wochen Follow-up jedoch nur in $33-53 \%$ der Fälle an [355, 356]. Bei kombinierter Anwendung von Bacitracin und Doxycyclin wurden VRE während zwei Wochen um $3 \mathrm{log} / \mathrm{g}$ Stuhl reduziert, stiegen aber danach wieder auf fast $8 \mathrm{log} / \mathrm{g}$ Stuhl an [353]. Ähnlich ungünstige Ergebnisse wurden mit der Kombination Bacitracin/Gentamicin erzielt
[357]. Auch nach Anwendung von Ramoplanin war nach anfänglicher Suppression der VRE in $90 \%$ nach weiteren zwei Wochen eine Rekolonisation mit VRE zu beobachten [358, 359]. Ebenso war die kurzzeitige Anwendung von Novobiocin in Kombination mit Tetracyclin oder Doxycyclin unwirksam [360].

Bei Kolonisation mit VRE ist die probiotische Therapie z. B. mit Lactobacillus rhamnosus GG (LGG) zur Herabsetzung der Kolonisationsdichte bis hin zur Eradikation erfolgversprechend [361]. In einer randomisierten kontrollierten Studie $(n=11)$ konnten VRE aus dem Gastrointestinaltrakt bei Nierenkranken nach vierwöchiger Gabe von LGG bei $91 \%$ im Vergleich zu $9 \%$ bei Placebo eradiziert werden [362]. Bei Kindern war VRE nach dreiwöchiger Behandlung (randomisierte single-blind placebokontrollierte Studie) mit LGG bei $62 \%(n=3232)$ der Kinder anstatt bei $24 \%(n=29)$ in der Placebogruppe signifikant weniger häufig nachweisbar [363]. Allerdings konnte in einem Setting mit hohem antibiotischen Selektionsdruck in einer Crossover-Studie durch Gabe von Probiotika (eine Mischung von Bifidobacterium-, Enterococcus- und Lactobacillus-Stämmen) ab dem Zeitpunkt der Aufnahme bis zur Entlassung die Akquisition multiresistenter Enterokokken nur tendenziell ( $25 \%$ vs. $20 \%$ in der Kontrolle) beeinflusst werden [364].

In einer randomisierten doppelblinden placebokontrollierten Studie wurde als sekundäres Outcome neben Machbarkeit und Verträglichkeit der 28-tägigen Gabe eines Probiotikums (Mischung von Bifidobacterium breve, B. infantis, B. longum, Lactobacillus acidophilus, L. plantarum, L. casei, L. bulgaricus und Streptococcus thermophilus) der Nachweis von VRE untersucht [365]. VRE waren zu Studienbeginn in der Placebogruppe bei 4/17 (24\%), in der Interventionsgruppe bei 5/19 (26\%) Patienten im Rektalabstrich nachweisbar. Nach vier Wochen gelang der VRE-Nachweis in der Placebogruppe bei 6/17 (38\%) Patienten, in der Interventionsgruppe bei 4/19 (21\%) Patienten.

Verschiedene Studien sprechen für das Potential von Probiotika zur Prävention einer mikrobiellen Fehlbesiedlung sowie zur Verhinderung der Ansiedlung von multiresistenten Bakterien wie VRE [124].
Sofern Probiotika allerdings bei immunsupprimierten Patienten oder kritisch kranken Patienten eingesetzt werden sollen, ist eine sorgfältige Risikoabwägung vorzunehmen [366-368]. Zudem ist nicht auszuschließen, dass probiotische E. faecium-Stämme das vanA-Gencluster akquirieren können [369].

Experimentell und epidemiologisch konnte gezeigt werden, dass Barnesiella $s p p$. im Darm die Clearance von VRE zu ermöglichen scheint, bzw. Resistenz gegenüber Besiedlung schaffen kann [370]. In ersten Versuchen mit FMT bei besiedelten Patienten konnten Davido et al. bei sieben von acht Patienten nach drei Monaten VRE nicht mehr nachweisen [371]. Hingegen fanden Sohn et al. bei drei VREkolonisierten Patienten nach FMT keine verkürzte Kolonisationsdauer [372]. Ergebnisse an größeren Patientengruppen und Langzeitergebnisse stehen jedoch noch aus, so dass der Einsatz nur unter Studienbedingungen und kritischer $\mathrm{Ab}$ wägung von Nutzen und Risiken erfolgen sollte.

\subsection{Antibiotic Stewardship}

Auf der Basis der vorliegenden Evidenz ist es nicht möglich, eine abschließende Wertung zur Rolle des Antibiotic Stewardships (ABS) zur Kontrolle von Kolonisation und Infektion mit VRE bei hospitalisierten Patienten zu treffen.

Während Exposition mit Vancomycin ein Risikofaktor für den Erwerb von VRE einzelner Patienten zu sein scheint [373], sind die Auswirkungen des VancomycinGebrauchs auf ganze Gruppen, wie zum Beispiel der Patientenpopulation eines Krankenhauses nicht gut dokumentiert.

In einer Reihe von Untersuchungen zeigte sich eine signifikante Assoziation zwischen einer Kolonisation oder Infektion mit VRE und einer vorausgegangenen Therapie mit Antibiotika [134, 223, 262, 373-375]. Während einige Studien eine Assoziation mit der Verwendung von Vancomycin fanden [262, 375], konnten andere keine Assoziation mit Vancomycin-Gebrauch nachweisen [376]. In einigen Untersuchungen wurde eine Assoziation nur mit anderen Antibiotika, nicht aber Vancomycin nachgewiesen [374, 377]. 
Leitlinien des CDC zur Kontrolle von VRE beinhalteten den umsichtigen Gebrauch von Vancomycin [378]. Erste Interventionen durch ABS in Bezug auf Vancomycin zur Reduzierung der VREAusbreitung bei hospitalisierten Patienten begannen in den frühen 1990er Jahren sowohl in endemischen $[379,380]$ als auch in epidemischen Settings [381]. Es fanden sich positive Resultate hinsichtlich der Reduzierung des Gebrauchs von Vancomycin, jedoch sehr kontroverse Ergebnisse bezüglich einer Reduzierung der Verbreitung von VRE. ABS-Programme einschließlich des kontrollierten Einsatzes verschiedener Antibiotikaklassen hatten hinsichtlich der Inzidenz von VRE bei hospitalisierten Patienten kontroverse Ergebnisse [382, 383].

Im Rahmen einer Interventionsstudie in einer hämatologischen Abteilung in Großbritannien wurde bei der empirischen Therapie von Fieber in der Neutropenie in einer ersten Phase Ceftazidim durch Piperacillin/Tazobactam ersetzt dies stand in Verbindung mit einem intensiven Schulungsprogramm zur Verbesserung der Händehygiene [331]. In einer zweiten Phase folgte die Wiedereinführung von Ceftazidim. Die erste Phase ging mit einer signifikanten Reduktion eines hyperendemischen VRE einher, die zweite Phase hingegen mit einem neuerlichen Anstieg des Vorkommens von VRE. Obwohl die Risikominimierung bezüglich eines Erwerbs von VRE teilweise den verbesserten Hygienemaßnahmen zuzuschreiben war, legt die Rückkehr des Problems nach Wiedereinführung von Ceftazidim nahe, dass Cephalosporine für den angestiegenen Erwerb unter hospitalisierten Patienten verantwortlich waren [331]. Eine Reduktion der VRE-Rate nach der Beschränkung von Breitspektrum-Cephalosporinen wurde darüber hinaus in einer intensivmedizinischen Abteilung für Brandverletzungen beobachtet [384].

Dagegen hatte eine Verbrauchsbeschränkung von Vancomycin bzw. Drittgenerations-Cephalosporinen wenig bis gar keinen Einfluss auf die VRE-Prävalenz im untersuchten Studienort [383, 385-388]. In einer Studie, die zur Bewertung des Einflusses eines eingeschränkten Gebrauchs von Vancomycin und Drittgenerations-Cephalosporinen bezüglich der VRE-Ausbreitung angelegt war, ging der
Gebrauch von Vancomycin nach der Einführung des ABS zunächst um $24 \% \mathrm{zu}$ rück, stieg aber bis zum Ende der Studie nach zehn Jahren wieder auf das Niveau vor der Intervention an. Der Gebrauch von Drittgenerations-Cephalosporinen verringerte sich dabei um $86 \%$. Die Prävalenz von VRE stieg während der Studie stetig und signifikant von $17 \%$ auf $30 \%$ an. Der Gebrauch von Clindamycin korrelierte signifikant mit dem Anstieg der VRE-Ausbreitung [385].

Ein systematisches Review umfasste 13 Studien (darunter keine randomisierten kontrollierten Studien), die bis März 2006 publiziert wurden [389]. Lediglich sieben der 13 Studien berichten über statistisch signifikante Reduktionen hinsichtlich des VRE-Erwerbs nach Interventionen. Die Ergebnisse reichten von einer Reduktion des VRE-Erwerbs um $82 \%$ bis zu einem Anstieg um $475 \%$. Die Studien, die sich auf spezifische Settings fokussierten (wie z.B. immunsupprimierte Patienten), erbrachten eher positive Resultate im Sinne einer Reduktion von VRE im Vergleich zu Studien, die auf das gesamte Klinikum ausgelegt waren. Die Art der Intervention, die epidemiologische Situation in der Einrichtung (z. B. endemisch oder epidemisch), das Studiendesign und die Dauer der Intervention standen in Relation zu deren Effektivität. Nur in sieben Studien wurde die Reduktion des VancomycinVerbrauchs als Einzelintervention implementiert, in allen anderen Untersuchungen wurden weitere Interventionen wie Infektionspräventionsmaßnahmen eingeführt oder der Gebrauch anderer Antibiotika reduziert [389]. Als Ergebnis wurden sowohl Prävalenz- als auch Inzidenzraten von Kolonisationen und/oder Infektionen gewählt. Das Studiendesign war so inhomogen, dass die Autoren davon Abstand nahmen, eine Meta-Analyse durchzuführen [389]. Von sechs zitierten Studien, bei denen die Intervention ausschließlich in einer Reduktion des Vancomycin-Gebrauchs bestand, fanden sich nur bei zwei Studien eine signifikante Reduktion von VRE-Infektion und/oder -Kolonisation. Dagegen gelang in fünf von sieben Studien, in denen weitere Präventionsmaßnahmen umgesetzt wurden, eine Reduktion der VRE-Infektion bzw. -Kolonisation [389, 390].
In einem aktuellen systematischen Review zum Einfluss der Implementation eines Antibiotic Stewardships auf die Inzidenz von VRE konnten innerhalb der Analyse drei Studien erfasst werden [391]. Angesichts der sehr unterschiedlichen Studien, welche hier verglichen wurden, lässt sich den Ergebnissen dieser Meta-Analyse im Hinblick auf die Effektivität eines „Antibiotic Stewardships“ auf die Verringerung der VRE-Rate kein konklusives Ergebnis entnehmen.

Eine Assoziation zwischen Antibiotikaexposition und VRE scheint sich abzuzeichnen. Welche Intervention einen Effekt auf VRE-Kolonisation oder -Infektion hat ist jedoch noch unklar. Interventionen zur Reduktion des Einsatzes von nicht indizierten Antibiotika sind sinnvoll und reduzieren die Selektion und Induktion von multiresistenten Erregern, wie auch VRE.

\section{Internationale Empfehlungen zum Umgang mit VRE}

Die vorliegenden internationalen Empfehlungen zur Prävention von Kolonisationen und Infektionen mit VRE weisen eine hohe Heterogenität auf.

Im Hinblick auf die zunehmende internationale Vernetzung der Gesundheitssysteme erscheint es sinnvoll, Empfehlungen zu Präventionsmaßnahmen im internationalen Vergleich zu betrachten.

Aboelela et al. verglichen sechs englischsprachige Guidelines hinsichtlich der Empfehlung zu Isolierungsmaßnahmen von MRE und konstatierten, dass erstens zumeist wenig Evidenz aus publizierten Studien die Grundlage für die Empfehlungen bildete und zweitens Empfehlungen zu VRE häufig analog zu bereits bestehenden Empfehlungen zum Umgang mit MRSA abgeleitet wurden [316].

Ein Konsens zum Umgang mit infizierten oder kolonisierten Patienten scheint nicht zu bestehen. Dies mag verschiedene Gründe haben, z. B. länderspezifische Strukturen des Gesundheitssytems oder unterschiedliche Prävalenz von VRE. Dennoch wurde der Prävention von VREInfektionen in vielen Ländern ausreichende Priorität eingeräumt, entsprechende Empfehlungen zur Prävention zu erarbeiten (• Tab. 9 in der Anlage). 


\section{Empfehlungen}

Vorrangiges Ziel der Empfehlungen ist die Prävention von nosokomialen Infektionen durch Enterokokken mit speziellen Antibiotikaresistenzen.

Aufgrund der unterschiedlichen Häufigkeit des Auftretens der Enterokokken mit speziellen Resistenzen einerseits und den Gegebenheiten in den jeweiligen Einrichtungen andererseits müssen die Maßnahmen individuell für die Einrichtung ausgewählt werden. Dies setzt Kenntnisse über die epidemiologische Situation und die eigenen Risikokollektive voraus.

Bei den vorliegenden Empfehlungen handelt es sich um Handlungsanweisungen, die in Abhängigkeit vom Ergebnis der zuvor durchgeführten infektionsepidemiologischen Analysen umgesetzt werden sollen. Hierfür werden Entscheidungskriterien angeboten, die die Auswahl und Anpassung der Maßnahmen erlauben.

\subsection{Erkennen, Erfassung und Bewertung von Infektionen durch Enterokokken}

Risikopopulationen für Infektionen durch Enterokokken im eigenen Patientengut können durch Infektionssurveillance auch unabhängig von speziellen Antibiotikaresistenzen erkannt werden. Die folgenden Maßnahmen zur Feststellung der Häufigkeit von Infektionen durch E. faecium sowie zur Identifikation von Risikopopulationen sind Gegenstand guter klinischer Praxis bzw. der Surveillance. Sie werden ohne Evidenzkategorie dargestellt.

\section{Die Kommission empfiehlt}

- in Zusammenarbeit von Klinikern, Krankenhaushygienikern und Mikrobiologen Art und Umfang der Enterokokkendiagnostik abzustimmen.

- die Patientenpopulationen oder Behandlungsgruppen zu identifizieren, in denen therapiebedürftige Infektionen durch E. faecium ${ }^{2}$ (unabhängig von der Antibiotikaresistenz) auftreten.

\footnotetext{
2 Infektionen durch E. faecium als alleinigem Erreger oder polymikrobielle Infektionen mit Beteiligung von E. faecium, bei denen E. faecium bewusst in die antibiotische Therapie einbezogen wurde.
}

- neben der Surveillance antibiotikaresistenter Enterokokken entsprechend $\$ 23$ IfSG eine kontinuierliche Bewertung der Häufigkeit von Infektionen durch E. faecium, die antibiotischer Therapie bedürfen, unabhängig von der Antibiotikaresistenz durchzuführen (z. B. durch Bewertung von Erregerstatistiken aus Blutkulturen).

- bei Zunahme von antibiotisch-therapiebedürftigen Infektionen durch $E$. faecium (unabhängig von der Antibiotikaresistenz) die Umsetzung der Maßnahmen der Basishygiene und des Antibiotic Stewardships zu prüfen und ggf. $\mathrm{zu}$ intensivieren sowie bei Verdacht auf einen epidemischen Zusammenhang entsprechende darüber hinausgehende Maßnahmen $\mathrm{zu}$ ergreifen.

\subsection{Empfehlungen zur Prävention von Infektionen durch VRE}

Die VRE-Kolonisation ist einer der wichtigsten Risikofaktoren für eine Infektion mit VRE.

Da kolonisierte Patienten eine Quelle für VRE sein können, wäre die Erkennung kolonisierter Patienten und entsprechende Umsetzung von Maßnahmen, die Übertragungen ausgehend von kolonisierten Patienten verhindern, ein sinnvolles Vorgehen. Ein solches Vorgehen erscheint in Regionen mit niedriger VREPrävalenz zielführend.

Demgegenüber stehen Erfahrungen und Berichte, dass in vielen Regionen Deutschlands bereits eine endemische Situation besteht, so dass schon bei der Aufnahme in die medizinische Einrichtung ein höherer Anteil an Patienten kolonisiert ist. In einem solchen Setting hat es sich in bisherigen Untersuchungen mit den dort umgesetzten Maßnahmen als kaum möglich erwiesen, Übertragungen nachhaltig zu verhindern. Andererseits treten Infektionen durch VRE nur in einem kleineren Teil kolonisierter Patienten und überwiegend in speziellen gefährdeten Patientengruppen auf. Die Empfehlungen hinsichtlich VRE konzentrieren sich folglich auf die Prävention von antibiotisch-therapiebedürftigen Infektionen.

\section{Die Kommission empfiehlt}

- eine Eingruppierung der Patientenpopulationen oder Behandlungsgruppen hinsichtlich ihres Risikos, Infektionen durch VRE zu erleiden, vorzunehmen (Kat. II).

- die konsequente Umsetzung der Basishygiene, solange in einer definierten Population keine antibiotisch-therapiebedürftigen VRE-Infektionen auftreten, unabhängig von der Anzahl der kolonisierten Patienten (Kat. II).

In Regionen oder Einrichtungen mit niedriger VRE-Prävalenz und ohne Auftreten von VRE-Infektionen kann die Zielsetzung der hygienischen Maßnahmen die Vermeidung von Transmissionen ausgehend von mit VRE besiedelten Patienten sein. Dann können über die Basishygiene hinausgehende Maßnahmen bei Nachweis einer Kolonisation umgesetzt werden. Die Zielsetzung der Maßnahmen sollte interdisziplinär abgestimmt werden.

Maßnahmen bei Auftreten einer antibiotisch-therapiebedürftigen Infektion durch VRE:

\section{Die Kommission empfiehlt}

- die Bewertung der VRE-Infektion hinsichtlich des Orts des Erwerbs der Infektion (nosokomial vs. aus einer anderen Klinik mitgebracht vs. ambulant erworben) (Kat. IV, IfSG).

- bei ambulant erworbenen VRE-Infektionen individuell festgelegte Maßnahmen umzusetzen, die eine Weiterverbreitung ausgehend von infizierten Patienten verhindern (Kat. II).

- bei Patienten mit VRE-Infektionen, die kurz zuvor aus einer anderen Klinik zuverlegt wurden, individuell festgelegte Maßnahmen umzusetzen, die eine Weiterverbreitung von VRE verhindern und die verlegende Klinik zu informieren, so dass dort ggf. weitere Maßnahmen umgesetzt werden können (Kat. II).

Erläuterung: Die Infektion dient als Sentinel-Ereignis, das auf ein mögliches Problem hinweisen kann. Damit solche Probleme nicht übersehen werden, ist es sinnvoll verlegende Kliniken zeitnah darüber zu informieren, dass von dort eine Infektion mitgebracht wurde. Der 
Informationsweg sollte zwischen den Kliniken abgestimmt werden.

- bei erstmaligem Auftreten einer nosokomialen Infektion in einer Patientenpopulation mit unbekannter VRE-Prävalenz die Prävalenz der VRE-Besiedelung in der betroffenen Population zu erfassen - mit dem Ziel, auf Basis der Ergebnisse eine Risikobewertung durchzuführen (Kat. II). Dies gilt auch, wenn der Infektionsfall in einer anderen Klinik diagnostiziert wurde, aber wahrscheinlich in der eigenen Klinik erworben worden war.

- konsequente Umsetzung der Basishygiene in der betroffenen Population, wenn im Rahmen der Prävalenzuntersuchung keine weiteren Fälle (besiedelte oder infizierte Patienten) detektiert werden (Kat. II).

- die Information von aufnehmenden Einrichtungen und niedergelassenen Ärzten bei der Verlegung, Überweisung oder Entlassung der Patienten gemäß Länderhygieneverordnung (Kat. IV).

Maßnahmen bei Auftreten einer oder mehrerer antibiotisch-therapiebedürftiger Infektionen in Populationen mit kolonisierten Patienten:

\section{Die Kommission empfiehlt}

- die Compliance mit Basishygiene, Bündeln zur Prävention Device-assoziierter Infektionen und Antibiotic Stewardship-Programmen zu überprüfen und bei ungenügender Compliance Maßnahmen zu deren Verbesserung zu ergreifen (Kat. IV).

- die Einführung, Schulung und Umsetzung eines Maßnahmenbündels, bestehend aus einer Auswahl (mindestens 2) der folgenden Komponenten (Beispiele für Maßnahmenbündel siehe Kapitel 3) (Kat. II):

- Screening

- Isolierung

- Antiseptisches Waschen

- Einbeziehung der Patienten in Hygienemaßnahmen

- Intensivierte Reinigung und Desinfektion der Umgebung.

- die Effizienz des Maßnahmenbündels anhand festzulegender geeigneter
Zielgrößen (z. B. zu unterschreitende Inzidenzdichte von VRE-Infektionen) mindestens durch Fortführung der Surveillance regelmäßig zu überprüfen (Kat. IV). Hierbei sollen die Zielgrößen bevorzugt auf eine Reduktion der Infektionen und nicht auf einen Erhalt des Status quo ausgelegt werden.

- bei Überschreiten der Zielwerte das Maßnahmenbündel zu überprüfen und ggf. zu erweitern (Kat. II).

- bei langfristiger Einhaltung der Zielgröße das Maßnahmenbündel weiter beizubehalten und die Surveillance fortzuführen und bei fehlendem Auftreten von nosokomialen antibiotischtherapiebedürftigen VRE-Infektionen über längere Zeiträume (z. B. mehr als ein Jahr) das Maßnahmenbündel schrittweise zu reduzieren (Kat. II).

- die Information von aufnehmenden Einrichtungen und niedergelassenen Ärzten bei der Verlegung, Überweisung oder Entlassung der Patienten gemäß Länderhygieneverordnung (Kat. IV).

\subsection{Mögliche Komponenten von Präventionsbündeln}

In Abschnitt 5.2 „Empfehlungen zur Prävention von Infektionen durch VRE“ wird genau definiert, unter welchen Umständen erweiterte Maßnahmenbündel, die über die Basishygiene hinausgehen, anzuwenden sind. Da die Maßnahmen zur Prävention von VRE-Transmissionen oder -Infektionen praktisch immer in Bündeln umgesetzt wurden, kann die Effizienz einzelner Maßnahmen nicht bewertet werden. Im Folgenden wird daher für die einzelnen Komponenten beschrieben, welche Anwendungsmethodik gewählt und von der KRINKO als maßgeblich für den Erfolg eingeschätzt wurde.

\subsubsection{Screening}

Die Kommission empfiehlt

Wenn Screening als Bestandteil eines Maßnahmenbündels ausgewählt wurde

- ein aktives VRE-Screening auf Risikopopulationen zu fokussieren (Kat. II).

- ggf. zusätzlich zum aktiven Screening das passive Screening aus Stuhlproben durchzuführen, die zur C. difficile-Diagnostik eingesandt werden (Kat. II).
- als Untersuchungsmaterial für das Screening Stuhlproben oder Rektalabstriche zu verwenden (Kat. II).

- zum sicheren Ausschluss einer neu erworbenen Besiedelung mindestens drei Proben an verschiedenen Tagen im Zeitraum von mindestens einer Woche (z. B. Tag 2, 5 und 7) zu entnehmen (Kat. II).

- zum Ausschluss einer fortbestehenden Besiedelung mindestens drei Proben an verschiedenen Tagen zu entnehmen (Kat. II). Aufgrund der langdauernden Besiedelung sollen längere Zeiten (z. B. eine Woche) zwischen den Kontrollabstrichen bevorzugt werden (Kat. II).

- die Labornachweismethode (Kultur oder Nukleinsäureamplifikationstechniken) nach Voraussetzungen vor Ort und der Fragestellung zu wählen, da kein nachgewiesener Vorteil für eine Methode besteht (Kat. III).

\subsubsection{Isolierung}

\section{Die Kommission empfiehlt}

Wenn Isolierung als Bestandteil eines Maßnahmenbündels ausgewählt wurde

- für die Isolierung von VRE-Trägern Zimmer mit eigener Nasszelle zu verwenden (Kat. II).

- eine Einzelunterbringung oder eine Unterbringung in einer Kohorte (Kat. II). Ob eine gemeinsame Kohortierung von VanA- und VanB-Trägern möglich ist, ist ungeklärt (Kat. III).

- keine Kohortierung von VRE-Patienten mit MRSA-Patienten oder mit anderen MRE-Patienten (Kat. IB).

- je nach Risikobewertung die Isolierung aller VRE-Träger oder die Isolierung von VRE-Trägern mit erhöhtem Risiko für eine Umgebungskontamination (z. B. ungenügende Compliance mit hygienischen Maßnahmen, akute Diarrhöen, Stuhlinkontinenz) (Kat. II).

- die Verwendung von Schutzkitteln und Handschuhen bei jedem Patientenkontakt (Kat. II).

\subsubsection{Antiseptisches Waschen}

Die Kommission empfiehlt

Wenn antiseptisches Waschen als Bestandteil eines Maßnahmenbündels ausgewählt wurde 
- antiseptische Waschungen zur Prävention von VRE-Infektionen auf Patientenpopulationen mit höherer ZVK-Anwendungsrate zu beschränken, weil bisherige Studien bevorzugt in Patientenpopulationen durchgeführt wurden, in denen ein hoher Patientenanteil einen ZVK hatte (Kat. IB).

- die Waschung mit chlorhexidinhaltigen Antiseptika ( $\geq 2 \%$ Chlorhexidingluconat) unter Beachtung der Nebenwirkungen durchzuführen (Kat. IB), für andere Antiseptika ist die Datenlage nicht ausreichend, so dass über deren Einsatz lokal nach Risikobewertung entschieden werden muss (Kat. III).

\subsubsection{Einbeziehung der Patienten in Hygienemaßnahmen \\ Die Kommission empfiehlt scheiden, da die Wirksamkeit eines auf VRE ausgerichteten ABS unge- klärt ist (Kat. III).}

Wenn die Einbeziehung von Patienten als Bestandteil eines Maßnahmenbündels ausgewählt wurde

- Schulung der Patienten und ggf. stichprobenartige Beobachtung der Händedesinfektion der Patienten, z. B. nach dem Toilettengang, vor der Nahrungsaufnahme und Medikamenteneinnahme (Kat. II).

- Einbeziehung der Patienten in Maßnahmen zur Reduktion der Kontamination in Sanitärräumen (Kat. II).

\subsubsection{Intensivierte Reinigung und Desinfektion der Umgebung}

Die Kommission empfiehlt

Wenn intensivierte Reinigung und Desinfektion als Bestandteil eines Maßnahmenbündels ausgewählt wurde

- eine mindestens tägliche Desinfektion der Patientenumgebung mit geprüft wirksamen Flächendesinfektionsmitteln (Kat. II).

- die Anpassung des Personalschlüssels des mit der Reinigung und Flächendesinfektion beauftragten Personals an erhöhte Anforderungen (Kat. II).

- die gezielte und wiederholte Schulung des Reinigungspersonals (Kat. II).

- eine regelmäßige Überprüfung der Qualität der desinfizierenden Reinigung (Kat. II).

- den Einsatz von Raumverneblern zusätzlich zur Schlussdesinfektion nur nach individueller Kosten-NutzenAnalyse zu erwägen, da hierfür noch keine ausreichenden Daten vorliegen (Kat. III).

\subsection{Weitere Maßnahmen zur Prävention von VRE-Infektionen oder-Transmissionen}

\subsubsection{Antibiotic Stewardship- Programme \\ Die Kommission empfiehlt}

- die Ausrichtung des ABS-Programms auf Prävention von VRE-Infektionen z. B. durch Reduktion des Vancomycin-Verbrauchs und weiterer Enterokokken-selektionierender Antibiotika wie Cephalosporine und Clindamycin nach lokalen Gegebenheiten zu ent-

\subsubsection{Maßnahmen zur Eradikation Die Kommission empfiehlt}

- keine Behandlung mit Antibiotika zur Eradikation einer Besiedelung (Kat. II).

- keinen Einsatz von Probiotika oder einer Stuhltransplantation mit dem Ziel der VRE-Eradikation bevor systematische Untersuchungen vorliegen (Kat. II).

\subsection{Empfehlungen zur Prävention von Infektionen durch LRE oder LVRE}

\section{Die Kommission empfiehlt}

- bei jeglichem Nachweis eines LRE oder LVRE bei einem Patienten die Isolierung des Patienten (oder ggf. der Kohorte in einem Zimmer mit eigener Nasszelle und Einhaltung von Barrieremaßnahmen (Kittel und Handschuhe) (Kat. II).

- die Bewertung der Infektion hinsichtlich des Orts des Erwerbs der Infektion (nosokomial vs. zuverlegter Fall vs. ambulant erworben) (Kat. IV, IfSG).

- bei zuverlegten Fällen die zuverlegende Einrichtung/Abteilung zu informieren, so dass dort weitere Maßnahmen umgesetzt werden können (ohne Kat.).
- die Surveillance zu erweitern durch mindestens eine der folgenden Maßnahmen:

- Systematische Testung aller Enterokokken-Isolate aus klinischen Materialien auf Linezolid-Resistenz (ohne Kat.).

- Untersuchung der LRE-Prävalenz durch Screening der Patienten.

- Finden sich keine weiteren besiedelten oder infizierten Patienten, so ist von einem Resistenzerwerb unter Therapie auszugehen und, abgesehen von der Isolierung des Patienten, sind keine weiteren Maßnahmen erforderlich (ohne Kat.).

- spätestens bei Auftreten eines zweiten Falles innerhalb von drei Monaten, der nosokomial erworben wurde, eine Prävalenzuntersuchung durchzuführen (ohne Kat.).

- bei Nachweis weiterer Patienten im Prävalenzscreening eine Ausbruchuntersuchung einzuleiten (Kat. IB).

- die Information von aufnehmenden Einrichtungen und niedergelassenen Ärzten bei der Verlegung, Überweisung oder Entlassung der Patienten gemäß Länderhygieneverordnung (Kat. IV).

\subsection{Empfehlungen zur}

Prävention von Infektionen

durch Enterokokken mit anderen

Antibiotikaresistenzen

\section{Die Kommission empfiehlt}

- bei Nachweis von Enterokokken mit high-level Gentamicin-Resistenz die konsequente Umsetzung der Basishygiene (Kat. II). Über die Basishygiene hinausgehende Maßnahmen sind nicht erforderlich.

- bei Nachweis von Enterokokken mit Resistenz gegenüber Tigecyclin oder Daptomycin die Isolate zu asservieren und an ein Referenzlabor, z. B. das NRZ für Enterokokken zu versenden, um die Resistenz bestätigen zu lassen (ohne Kat.).

- bei Nachweis von Enterokokken mit Resistenz gegenüber neueren Reserveantibiotika die Antibiotikaempfindlichkeitsprüfung aller Enterokokken-Isolate der gleichen Spezies aus klinischen Materialien um die ent- 


\begin{tabular}{|c|c|}
\hline \multicolumn{2}{|c|}{ Abkürzungen } \\
\hline$A B$ & Antibiotikum \\
\hline$A B S$ & Antibiotic Stewardship \\
\hline AFLP & Amplified Fragment-Length Polymorphism \\
\hline $\mathrm{CHX}$ & Chlorhexidin \\
\hline$C D C$ & Centers for Disease Control and Prevention \\
\hline $\mathrm{Cl}$ & Konfidenzintervall \\
\hline CLSI & Clinical and Laboratory Standards Institute \\
\hline$E S B L$ & Extended Spectrum Beta-Lactamase \\
\hline EUCAST & European Committee on Antimicrobial Susceptibility Testing \\
\hline FMT & Fäkale Mikrobiota-Transplantation \\
\hline $\mathrm{H}_{2} \mathrm{O}_{2}$ & Wasserstoffperoxid \\
\hline$H A$ & Hospital-Assoziiert \\
\hline$H G T$ & Horizontaler Gentransfer \\
\hline HL-GRE & High-level Gentamicin-resistente Enterokokken \\
\hline IfSG & Infektionsschutzgesetz \\
\hline ITS & Intensivstation \\
\hline KBE & Koloniebildende Einheit \\
\hline LGG & Lactobacillus rhamnosus GG \\
\hline LRE & Linezolid-resistente Enterokokken \\
\hline LSE & Linezolid-sensible Enterokokken \\
\hline LVRE & Linezolid Vancomycin-resistente Enterokokken \\
\hline LVSE & Linezolid Vancomycin-sensible Enterokokken \\
\hline $\begin{array}{l}\text { MALDI } \\
\text { TOFMS }\end{array}$ & Matrix-Assistierte Laser-Desorption-Ionisierung Time of Flight Massenspektrometrie \\
\hline MHK & Minimale Hemmstoffkonzentration \\
\hline MP & Medizinprodukt \\
\hline MRE & Multiresistente Erreger \\
\hline MLST & Multilocus-Sequenztypisierung \\
\hline MLVA & Multiple Locus Variable number of tandem repeats Analysis \\
\hline MRSA & Methicillin-resistenter S. aureus \\
\hline NAAT & Nukleinsäureamplifikationstechnik \\
\hline $\mathrm{NI}$ & Nosokomiale Infektion \\
\hline NRZ & Nationales Referenzzentrum \\
\hline OP-KISS & $\begin{array}{l}\text { Modul „Postoperative Wundinfektionen“ des Krankenhaus-Infektions-Surveillance- } \\
\text { Systems }\end{array}$ \\
\hline$O R$ & Odds Ratio \\
\hline$P$ & Patient \\
\hline PT & Patiententage \\
\hline$P C R$ & Polymerase-Kettenreaktion \\
\hline$R R$ & Risk Ratio \\
\hline$V R$ & Vancomycin-Resistenz \\
\hline VRE & Vancomycin-resistente Enterokokken \\
\hline VSE & Vancomycin-sensible Enterokokken \\
\hline$Z V K$ & Zentraler Venenkatheter \\
\hline
\end{tabular}

sprechende Antibiotikaresistenz zu erweitern (ohne Kat.).

- spätestens bei Auftreten eines zweiten Falles von Enterokokken mit Resistenz gegenüber demselben neuen Reserveantibiotikum innerhalb von drei Monaten eine Prävalenzuntersuchung durchzuführen (ohne Kat.).

- bei Nachweis weiterer Patienten im Prävalenzscreening eine Ausbruchuntersuchung einzuleiten (ohne Kat.).

\subsection{Begleitende Maßnahmen im Rahmen der Umsetzung}

\section{Die Kommission empfiehlt}

- die Information von betroffenen Patienten und Angehörigen über das gewählte Vorgehen in der Einrichtung und wie sie zum Gelingen der Maßnahmen beitragen können (Kat. II).

- Kommunikation über die unterschiedlichen Maßnahmenpakete innerhalb einer Einrichtung und zwischen Einrichtungen (Kat. II) und ggf. den Austausch über die gewählten Maßnahmen innerhalb der Netzwerke.

Interessenkonflikt. Diese Empfehlungen wurden ehrenamtlich und ohne Einflussnahme kommerzieller Interessengruppen im Auftrag der Kommission für Krankenhaushygiene und Infektionsprävention erarbeitet von einer Arbeitsgruppe bestehend aus PD Dr. Christian Brandt, Prof. Dr. Axel Kramer, Dr. Annegret Krenz-Weinreich, Prof. Dr. Evelina Tacconelli, Prof. Dr. Heike von Baum und Prof. Dr. Constanze Wendt (Leiterin der Arbeitsgruppe). Vom Robert Koch-Institut waren beteiligt: Prof. Dr. Guido Werner, Prof. Dr. Mardjan Arvand, Dr. Eva Feuerhahn und Melanie Brunke. Die Empfehlung wurde durch die Arbeitsgruppe vorbereitet und nach ausführlicher Diskussion in der Kommission abgestimmt. 


\section{Anlagen}

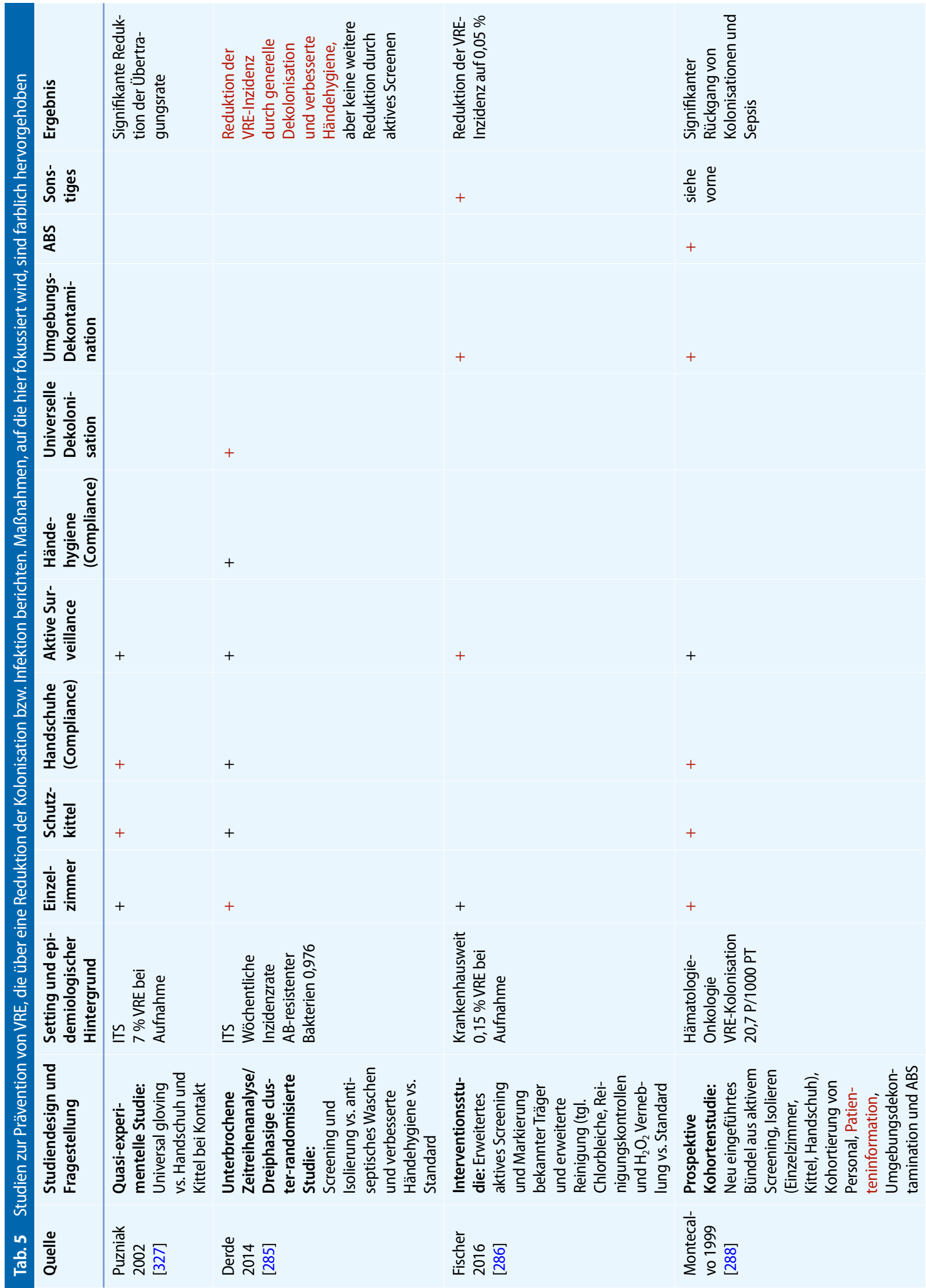




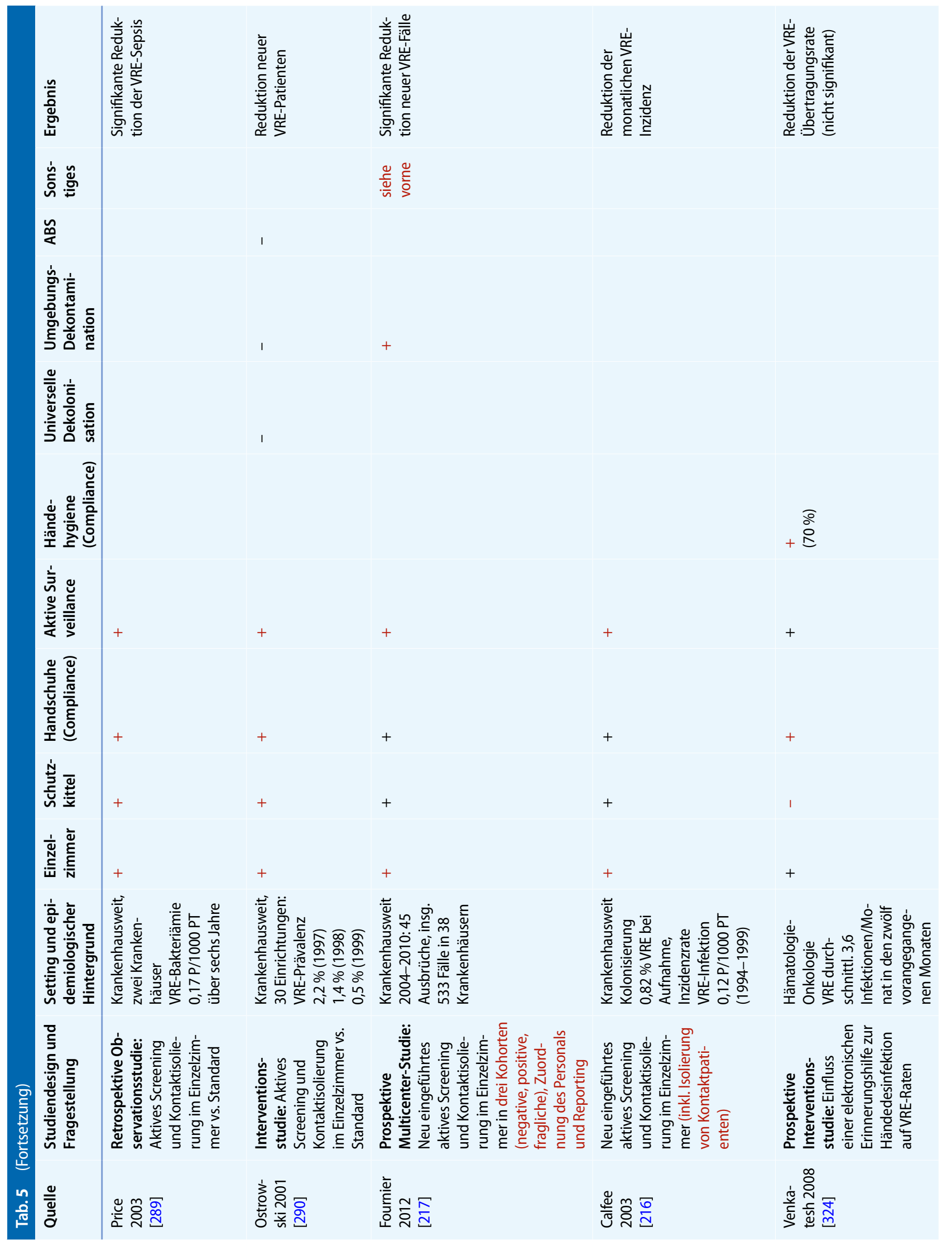




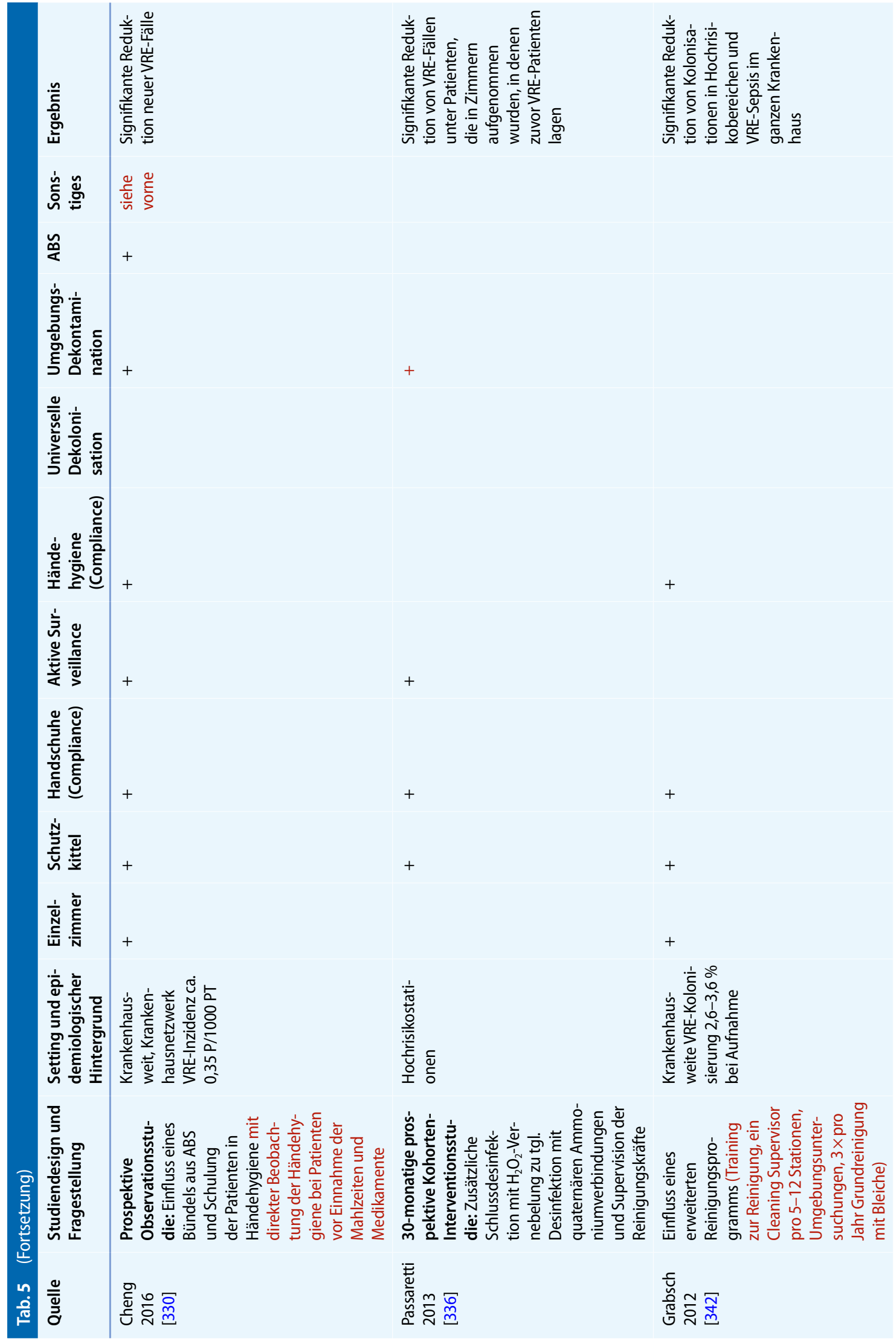




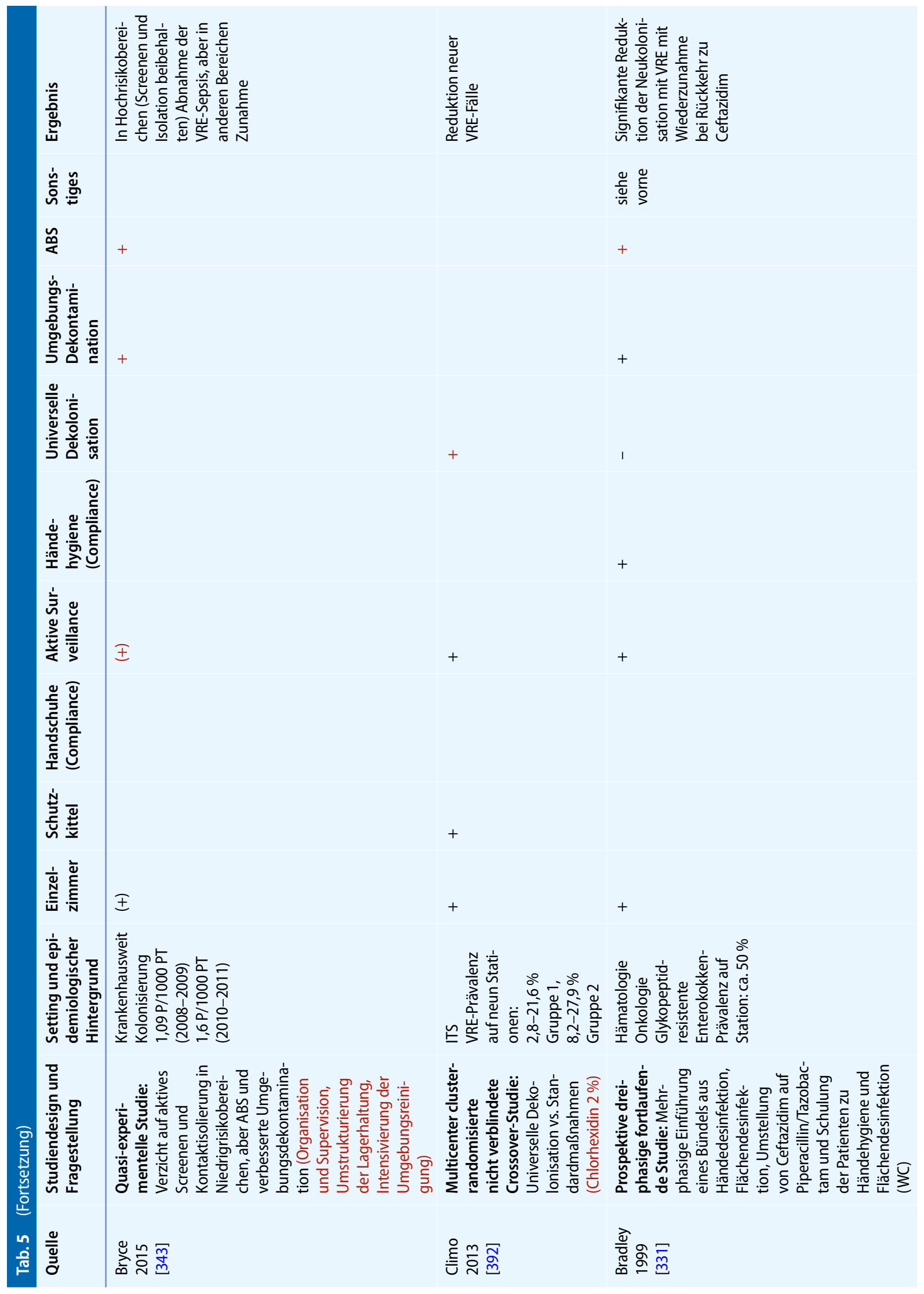




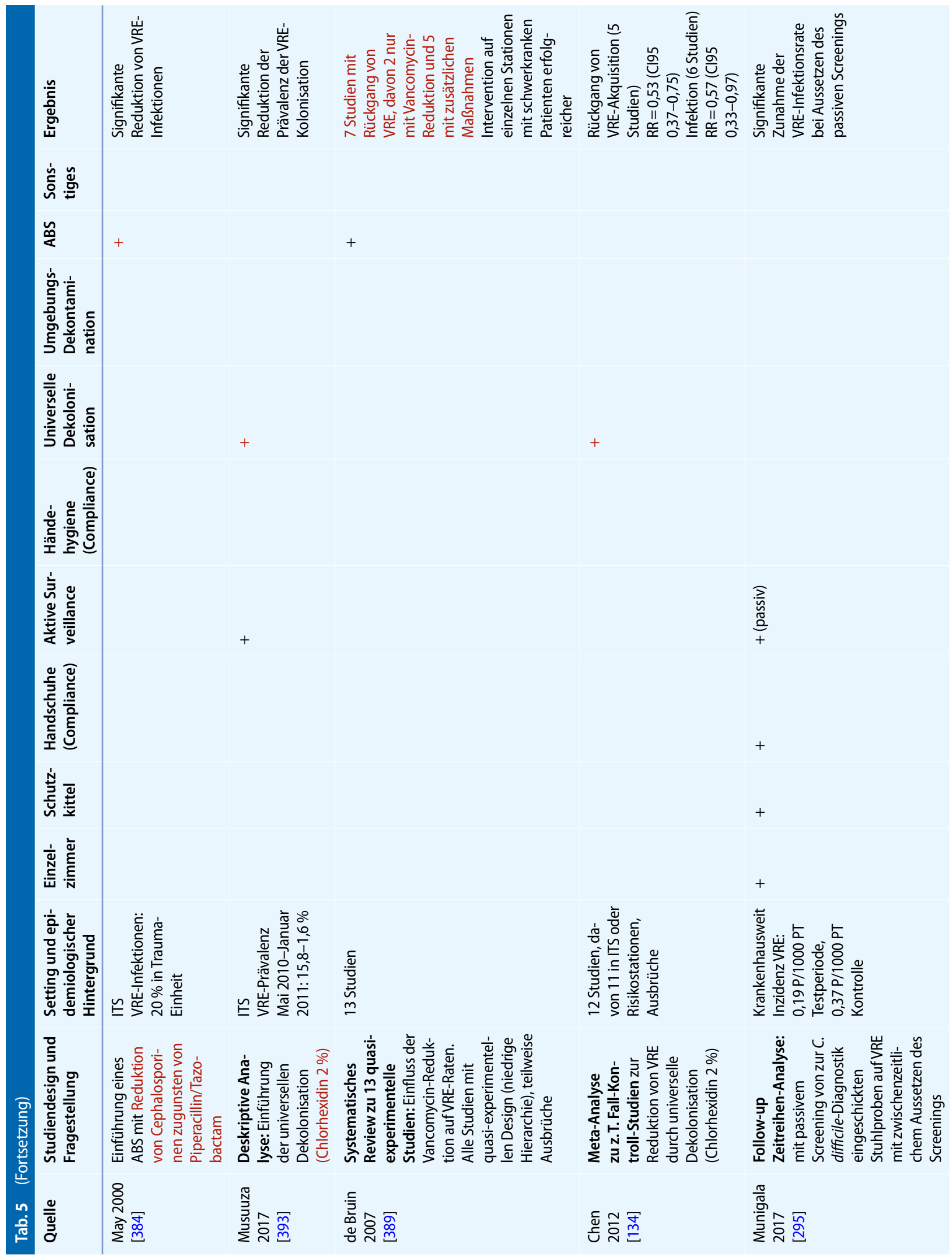




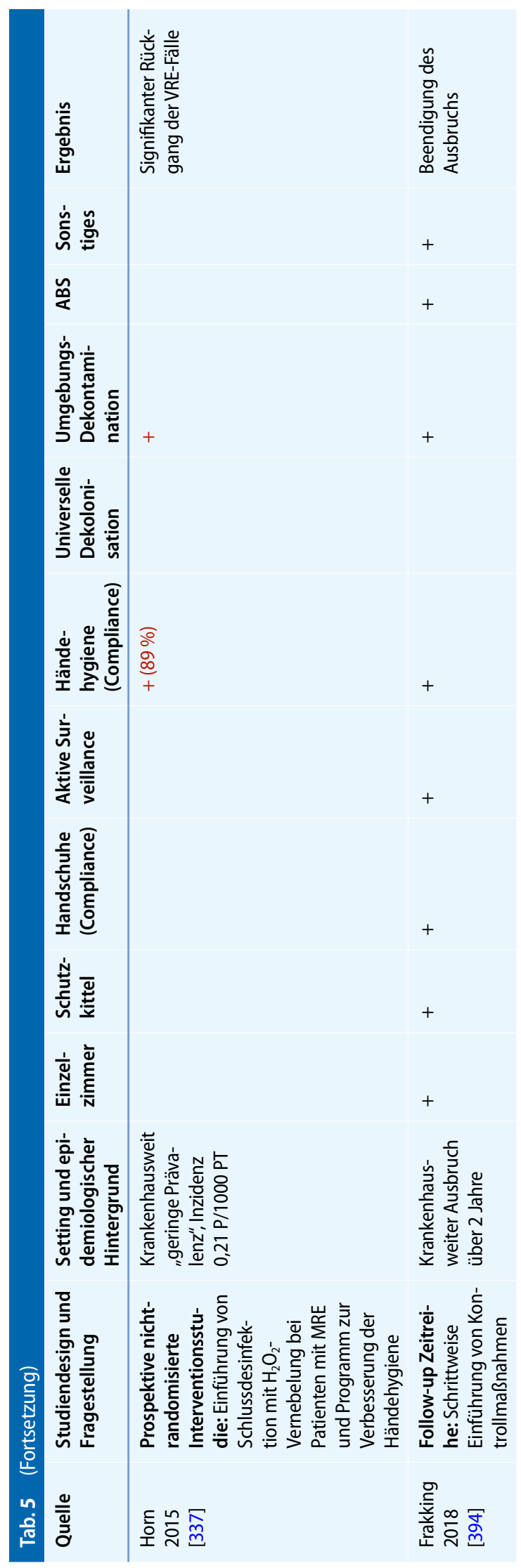




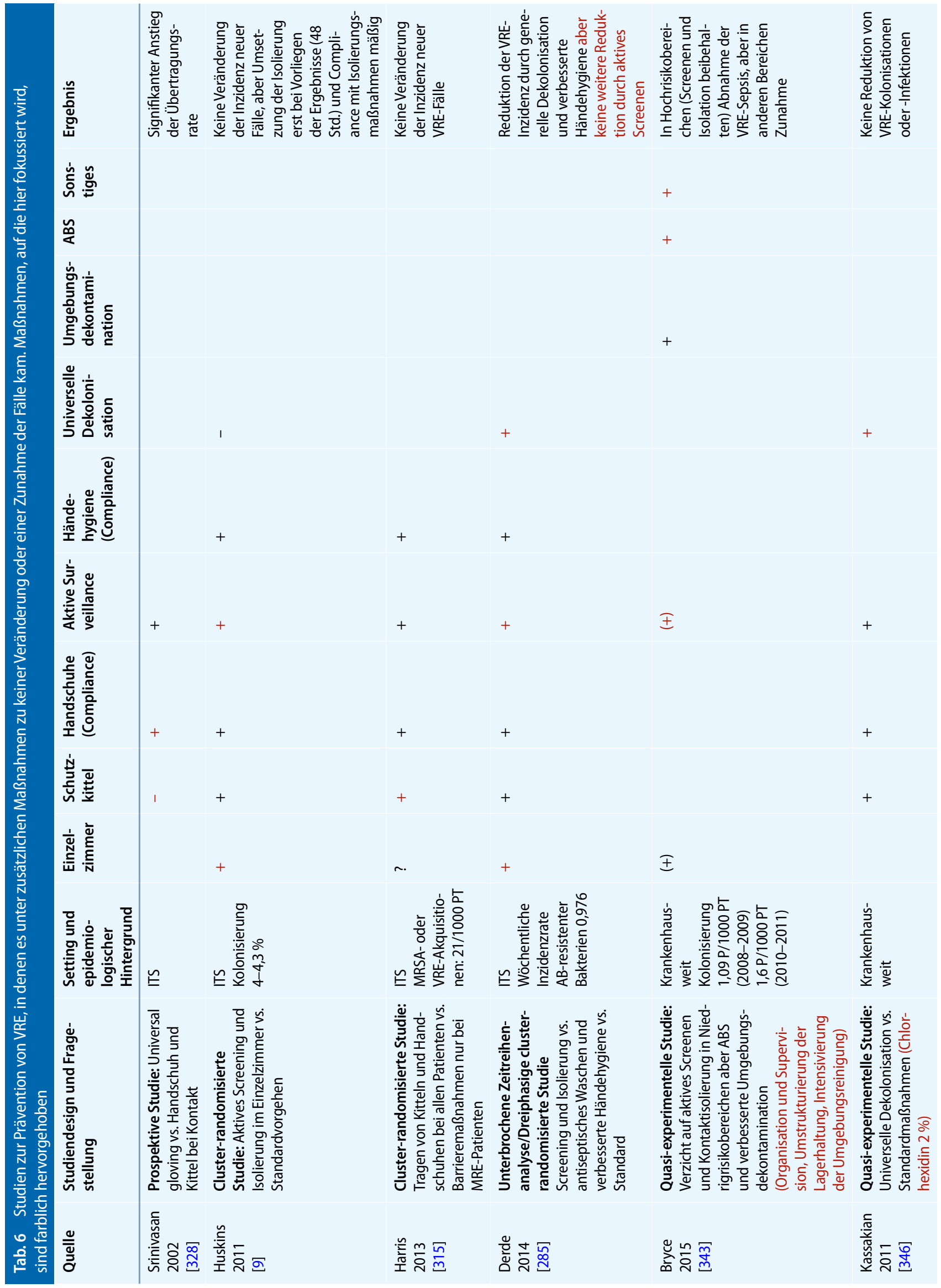




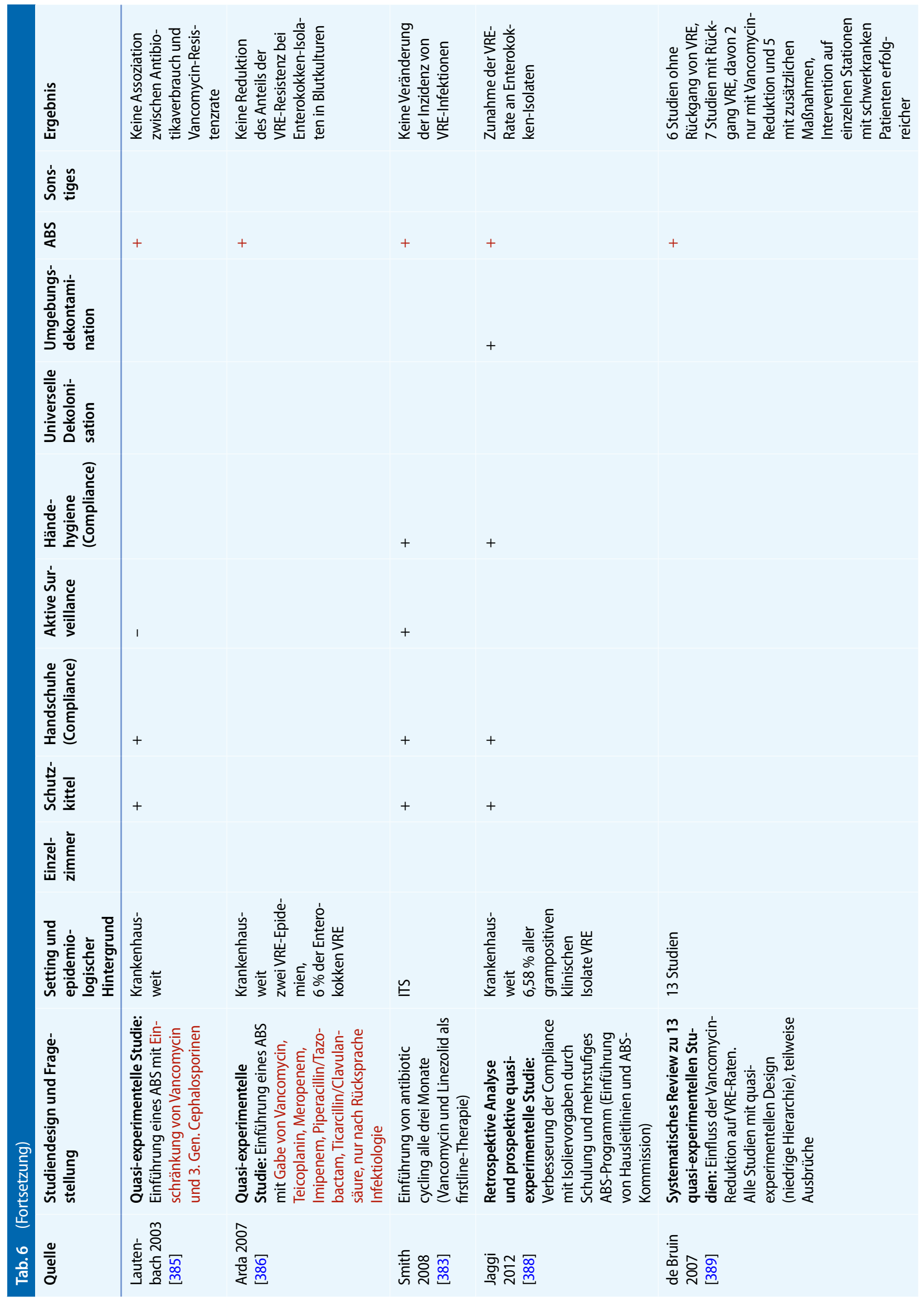




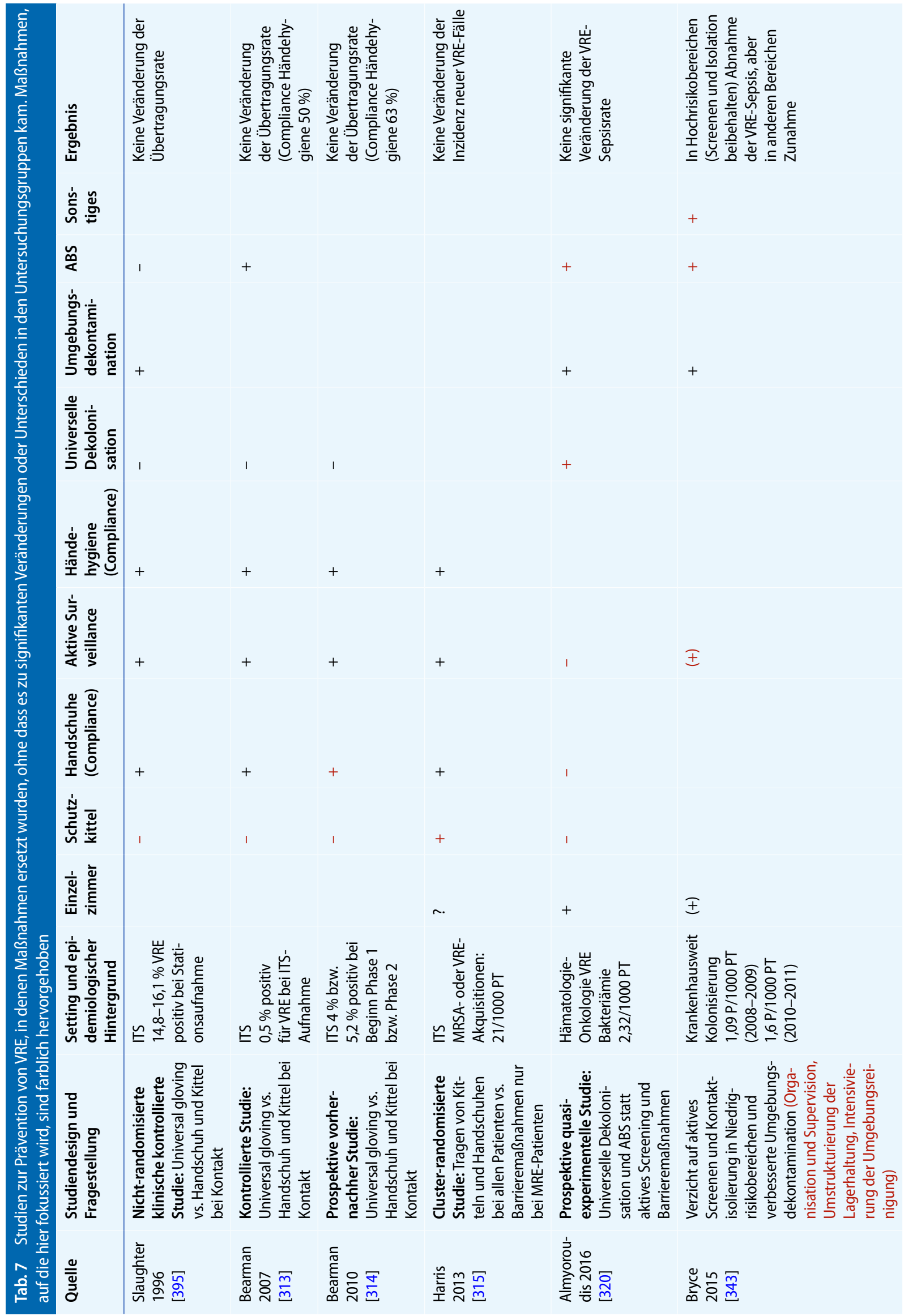




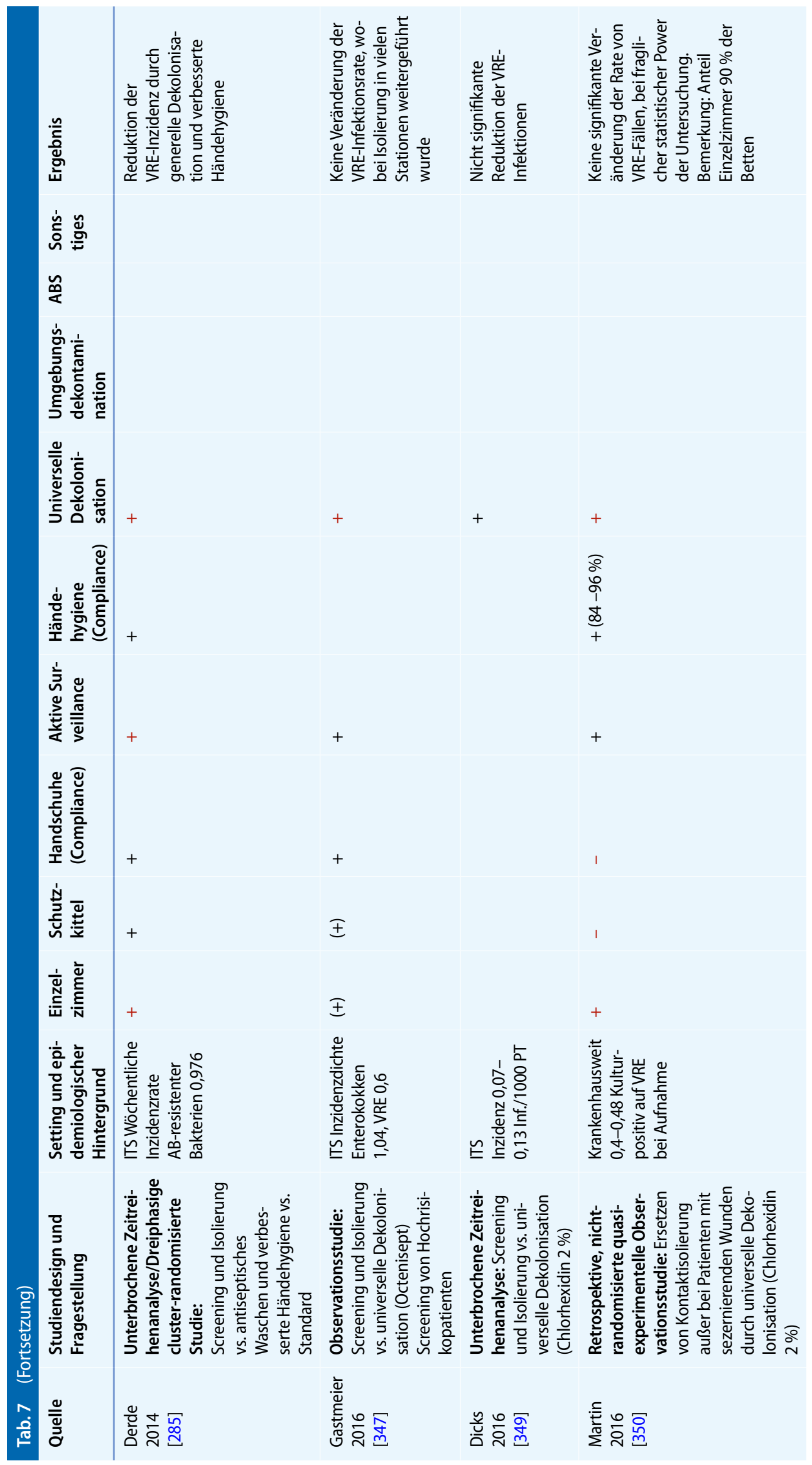




\section{Bekanntmachungen - Amtliche Mitteilungen}

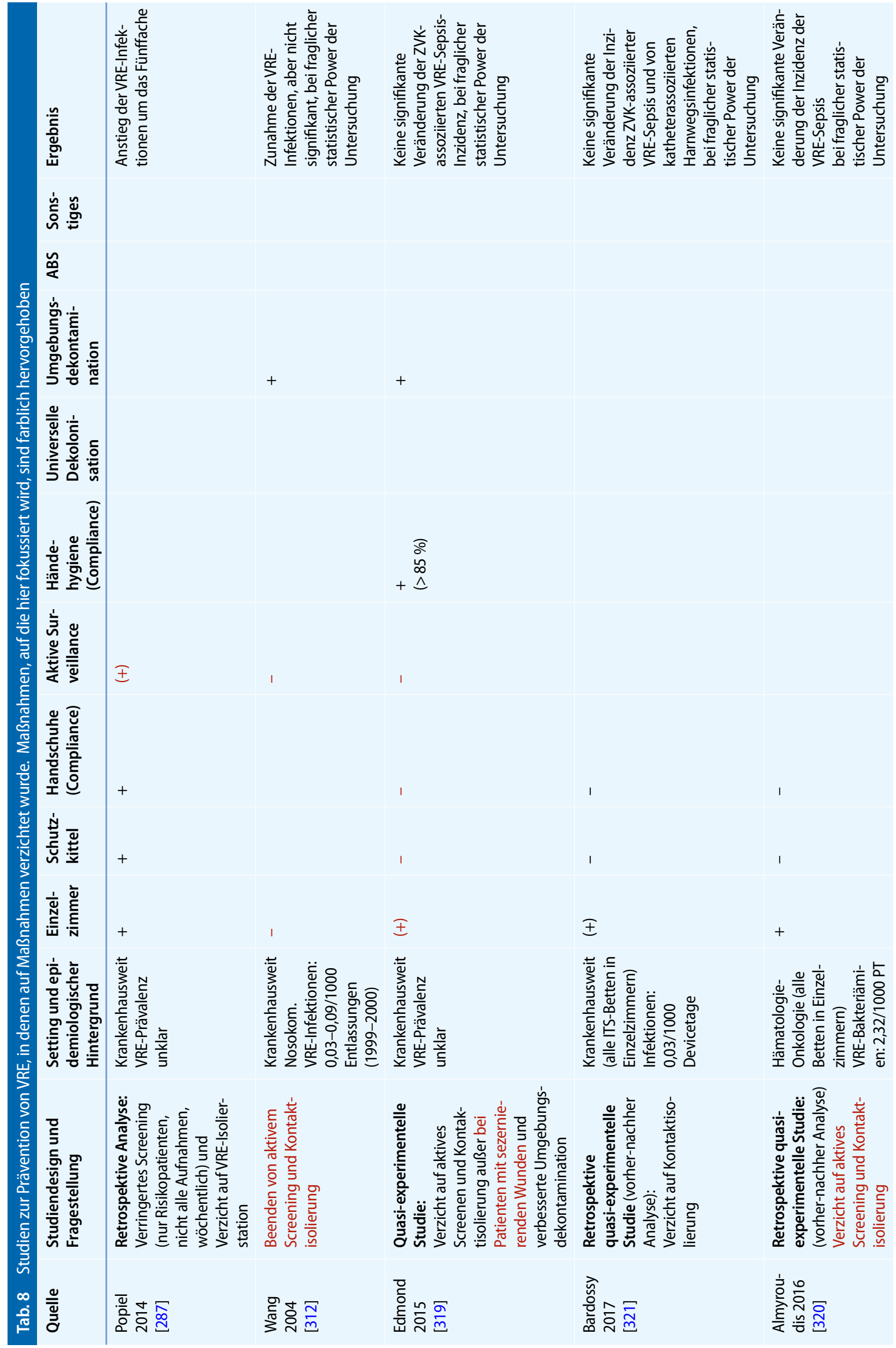




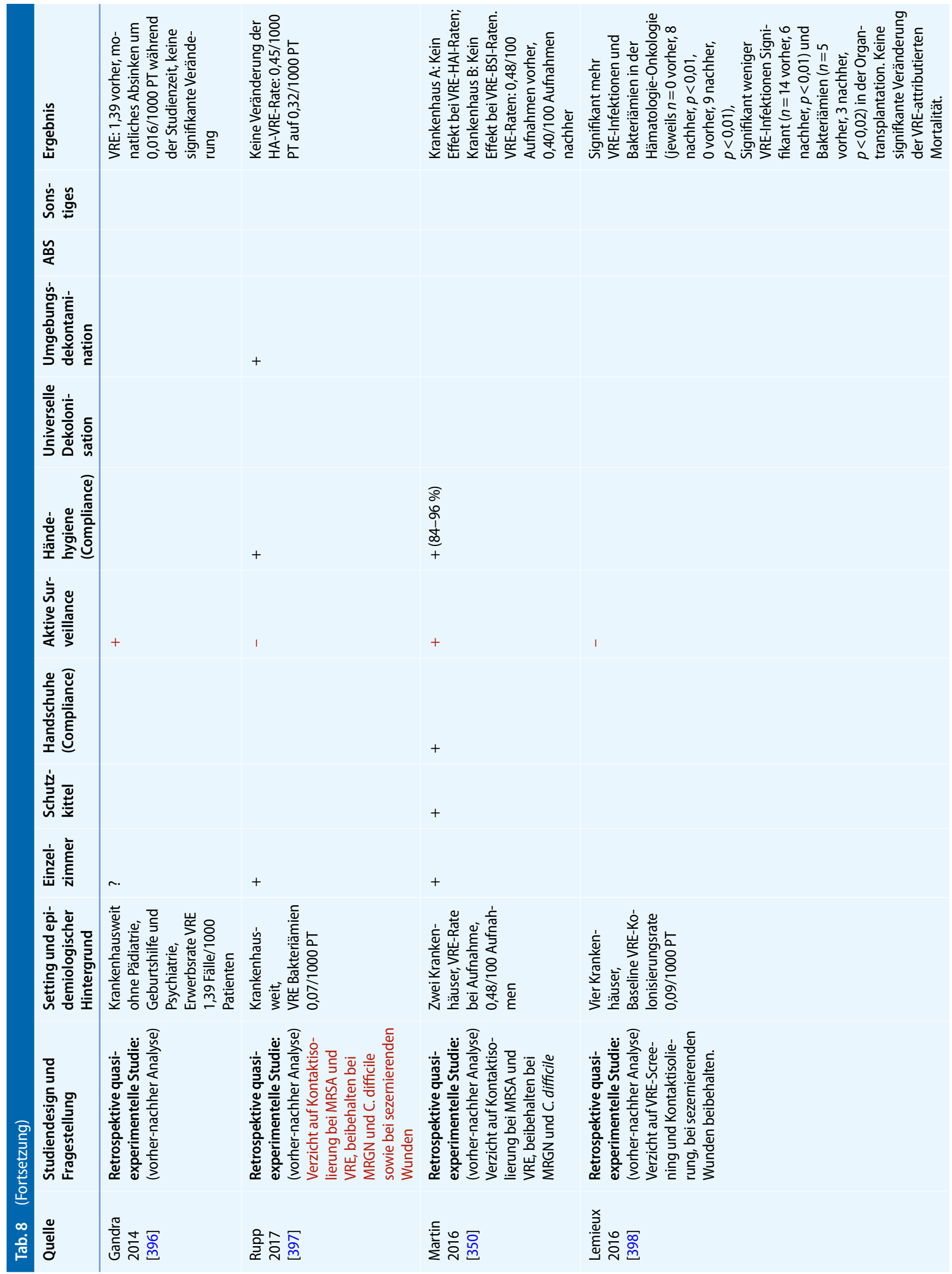


Tab.9 Vergleich internationaler Empfehlungen zur Prävention von Kolonisation oder Infektion mit VRE

\begin{tabular}{|c|c|c|c|c|c|c|}
\hline & $\begin{array}{l}\text { USA } \\
\text { Muto et al. (SHEA), } \\
2003 \text { [399] }\end{array}$ & $\begin{array}{l}\text { USA } \\
\text { Siegel et al. } \\
\text { (HICPAC), } 2006 \\
{[400]}\end{array}$ & $\begin{array}{l}\text { Australien } \\
\text { SA Health, } 2017 \\
\text { [401] }\end{array}$ & $\begin{array}{l}\text { Frankreich } \\
\text { Lepelletier et al., } \\
2015 \text { [402] }\end{array}$ & $\begin{array}{l}\text { Niederlande } \\
\text { Kluytmans et al., } \\
2005 \text { [403] }\end{array}$ & $\begin{array}{l}\text { Großbritannien } \\
\text { Cookson et al., } \\
2006[404]\end{array}$ \\
\hline Titel & $\begin{array}{l}\text { SHEA Guideline for } \\
\text { Preventing Nosoco- } \\
\text { mial Transmission of } \\
\text { Multidrug-Resistant } \\
\text { Strains of Staphylo- } \\
\text { coccus aureus and } \\
\text { Enterococcus }\end{array}$ & $\begin{array}{l}\text { Management of } \\
\text { multidrug-resist- } \\
\text { ant organisms } \\
\text { in health care } \\
\text { settings }\end{array}$ & $\begin{array}{l}\text { Management of } \\
\text { Patients with Van- } \\
\text { comycin-resistant } \\
\text { Enterococci (VRE) - } \\
\text { Clinical Guideline }\end{array}$ & $\begin{array}{l}\text { French recom- } \\
\text { mendations for } \\
\text { the prevention of } \\
\text { emerging exten- } \\
\text { sively drug-resist- } \\
\text { ant bacteria (eXDR) } \\
\text { cross-transmission }\end{array}$ & $\begin{array}{l}\text { Dutch Guideline for } \\
\text { Preventing Nosoco- } \\
\text { mial Transmission } \\
\text { of Highly Resistant } \\
\text { Microorganisms } \\
\text { (HRMO) }\end{array}$ & $\begin{array}{l}\text { Guidelines for } \\
\text { the control of glyco- } \\
\text { peptide-resistant } \\
\text { Enterococci } \\
\text { in hospitals }\end{array}$ \\
\hline $\begin{array}{l}\text { Empfehlung } \\
\text { zum Screening }\end{array}$ & $\begin{array}{l}\text { Aufnahmescreening } \\
\text { und periodisches } \\
\text { wöchentliches } \\
\text { Screening für } \\
\text { Patienten mit hohem } \\
\text { Kolonisationsrisiko. } \\
\text { Risikofaktoren: } \\
\text { ITS/Antibiotikathe- } \\
\text { rapie/Grunderkran- } \\
\text { kung/Dauer des } \\
\text { Krankenhausaufent- } \\
\text { haltes. } \\
\text { Frequenz des Scree- } \\
\text { nings abhängig von } \\
\text { der Prävalenz }\end{array}$ & $\begin{array}{l}\text { Aktives } \\
\text { Screening } \\
\text { zu erwägen in } \\
\text { Situationen, wo } \\
\text { andere Maßnah- } \\
\text { men ineffektiv } \\
\text { waren. } \\
\text { Risikopatienten } \\
\text { und Risikosta- } \\
\text { tionen sollen } \\
\text { lokal festgelegt } \\
\text { werden. }\end{array}$ & $\begin{array}{l}\text { Aufnahme- } \\
\text { screening auf ITS } \\
\text { (adult), } \\
\text { auf renalen oder } \\
\text { Transplantations- } \\
\text { einheiten, } \\
\text { in Abh. von der } \\
\text { epidemiologischen } \\
\text { Lage auf neonat., } \\
\text { pädiatr. ITS bzw. } \\
\text { auf hämatologisch- } \\
\text { onkologischen ITS. } \\
\text { Periodisches } \\
\text { Screening } \\
\text { (wöchentlich und } \\
\text { bei Entlassung) }\end{array}$ & $\begin{array}{l}\text { Aufnahme- } \\
\text { screening von } \\
\text { Patienten, die } \\
\text { aus ausländischem } \\
\text { Krankenhäusern } \\
\text { kommen/in } \\
\text { ausländischen } \\
\text { Krankenhäusern } \\
\text { innerhalb des } \\
\text { letzten Jahres } \\
\text { versorgt wurden/ } \\
\text { Trägerstatus hatten } \\
\text { oder Kontaktpati- } \\
\text { ent waren }\end{array}$ & $\begin{array}{l}\text { Aktives Screening } \\
\text { von Kontaktpatien- } \\
\text { ten, ITS-Patienten, } \\
\text { die von einem ande- } \\
\text { ren niederländischen } \\
\text { Krankenhaus von } \\
\text { einer Station mit be- } \\
\text { kanntem Ausbruch } \\
\text { verlegt wurden, } \\
\text { ITS-Patienten, die } \\
\text { in einem ausländi- } \\
\text { schen Krankenhaus } \\
\text { in den letzten zwei } \\
\text { Monaten behandelt } \\
\text { wurden. }\end{array}$ & $\begin{array}{l}\text { Screening in Aus- } \\
\text { bruchsituationen }\end{array}$ \\
\hline $\begin{array}{l}\text { Räumliche } \\
\text { Unterbringung }\end{array}$ & keine Aussage & Einzelzimmer & $\begin{array}{l}\text { „Acute health care } \\
\text { facility": bevorzugt } \\
\text { Einzelzimmer mit } \\
\text { eigener Nasszelle; } \\
\text { "small acute health } \\
\text { care hospital“ oder } \\
\text { anderes Setting: } \\
\text { variabel }\end{array}$ & Einzelzimmer & Einzelzimmer & $\begin{array}{l}\text { bei Diarrhoe bzw. } \\
\text { Inkontinenz bevor- } \\
\text { zugt Einzelzimmer }\end{array}$ \\
\hline $\begin{array}{l}\text { Über Basis- } \\
\text { hygiene hinaus- } \\
\text { gehende } \\
\text { Maßnahmen }\end{array}$ & $\begin{array}{l}\text { Kontakt- oder Ko- } \\
\text { hortenisolierung, } \\
\text { - bei Betreten des } \\
\text { Zimmers von ko- } \\
\text { lonisierten/infizi- } \\
\text { zierten Patienten } \\
\text { Anlegen von } \\
\text { Handschuhen } \\
\text { - Anlegen von Kit- } \\
\text { teln bei Patienten- } \\
\text { und Umgebungs- } \\
\text { kontakt } \\
\text { - bei Patienten mit } \\
\text { ausstehendem } \\
\text { Screening- } \\
\text { Ergebnis: gene- } \\
\text { relle Kittel- und } \\
\text { Handschuhpflicht } \\
\text { oder generelle } \\
\text { Handschuhpflicht }\end{array}$ & $\begin{array}{l}\text { - Kittel und } \\
\text { Handschuhe } \\
\text { bei Betreten } \\
\text { des Zimmers/ } \\
\text { Nische } \\
\text { - Ablegen von } \\
\text { Kittel und } \\
\text { Handschuhen } \\
\text { vor Verlassen } \\
\text { des Zimmers/ } \\
\text { Nische }\end{array}$ & $\begin{array}{l}\text { Handschuhe bei } \\
\text { Patienten- und } \\
\text { Oberflächen- } \\
\text { kontakt bis zum } \\
\text { Verlassen des } \\
\text { Patientenzim- } \\
\text { mers bzw. Pati- } \\
\text { entenbereichs } \\
\text { - Wechsel der } \\
\text { Handschuhe } \\
\text { bei Tätigkeiten } \\
\text { an demselben } \\
\text { Patienten } \\
\text { - flüssigkeitsdich- } \\
\text { te Einmalkittel/ } \\
\text {-schürze bei } \\
\text { direktem Pati- } \\
\text { entenkontakt, } \\
\text { Patientenumge- } \\
\text { bung und bei } \\
\text { Körperflüssig- } \\
\text { keiten }\end{array}$ & $\begin{array}{l}\text { - Kontaktiso- } \\
\text { lation bis zur } \\
\text { Diagnose und } \\
\text { darüber hinaus } \\
\text { - Einmalschürzen } \\
\text { - Einmalhand- } \\
\text { schuhe } \\
\text { - Händedesin- } \\
\text { fektion }\end{array}$ & $\begin{array}{l}\text { Kontaktisolation } \\
\text { nach Diagnose } \\
\text { bis zur Entlas- } \\
\text { sung }\end{array}$ & keine Aussage \\
\hline
\end{tabular}


USA 2003 [399]

\begin{tabular}{ll}
\hline Umgang mit & patientenbezogene \\
Medizinproduk- & Benutzung von unkri- \\
ten (MP) und & tischen Instrumenten \\
Bedarfs-gegen- & oder systematische \\
ständen & $\begin{array}{l}\text { Aufbereitung nach } \\
\text { jeder Benutzung }\end{array}$
\end{tabular}
Spezielle
Zuordnung von
Personal zu VREPatienten (nichtAusbruchsituation)

Empfehlung zur täglichen Ganzkörperwaschung mit CHX

Sonstiges

\section{keine Aussage}

keine Aussage

keine Aussage

\section{USA} [400]

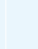

Siegel et al. (HICPAC), 2006

patientenbezogene Verwendung von unkritischen Instrumenten 


\section{Literatur}

1. DiazGranados CA, Zimmer SM, Mitchel K, Jernigan JA (2005) Comparison of mortality associated with vancomycin-resistant and vancomycin-susceptible enterococcal bloodstream infections: a meta-analysis. Clin Infect Dis 41(3):327-333

2. Spera RV Jr., Farber BF (1992) Multiply-resistant Enterococcus faecium. The nosocomial pathogen of the 1990s. JAMA 268(18):2563-2564

3. Acar J, Casewell M, Freeman J, Friis C, Goossens H (2000) Avoparcin and virginiamycin as animal growth promoters: a plea for science in decisionmaking. Clin Microbiol Infect 6(9):477-482

4. Marshall BM, Levy SB (2011) Food animals and antimicrobials: impacts on human health. Clin Microbiol Rev 24(4):718-733

5. Remschmidt C, Schröder C, Behnke M, Gastmeier P, Geffers C, Kramer TS (2018) Continuous increase of vancomycin resistance in enterococci causing nosocomial infections in Germany- 10 years of surveillance. Antimicrob Resist Infect Control 7(1):54

6. Bundesamt für Verbraucherschutz und Lebensmittelsicherheit (BVL), Paul-Ehrlich-Gesellschaft für Chemotherapie e.V. (PEG) (2016) GERMAP 2015. Bericht über den Antibiotikaverbrauch und die Verbreitung von Antibiotikaresistenzen in der Human- und Veterinärmedizin in Deutschland. Antiinfectives Intelligence: Rheinbach. http://www.p-e-g.org/econtext/germap. Zugegriffen: 22. März 2018

7. World Health Organization (WHO) (2017) Global priority list of antibiotic-resistant bacteria to guide research, discovery, and development of new antibiotics. http://www.who.int/ entity/medicines/publications/WHO-PPLShort_Summary_25Feb-ET_NM_WHO.pdf. Zugegriffen: 22. März 2018

8. von Baum H, Dettenkofer M, Fahr AM, Heeg P, Wendt C (2006) Umgang mit Patienten mit Glykopeptid-resistenten Enterokokken (GRE)/ Vancomycin-resistenten Enterokokken (VRE). Hyg Med 31(1):30-32

9. Huskins WC, Huckabee CM, O'grady NP et al (2011) Intervention to reduce transmission of resistant bacteria in intensive care. $\mathrm{N}$ Engl $\mathrm{J}$ Med 364(15):1407-1418

10. Mutters NT, Mersch-Sundermann V, Mutters $\mathrm{R}$, Brandt C, Schneider-Brachert W, Frank U (2013) Kontrolle von Vancomycin-resistenten Enterokokken im Krankenhaus. Epidemiologischer Hintergrund und klinische Relevanz. Dtsch Arztebl Int 110(43):725-731

11. Sprague E, Reynolds S, Brindley P (2016) Patient isolation precautions: are they worth it? Can Respir J. https://doi.org/10.1155/2016/5352625

12. Kommission für Krankenhaushygiene und Infektionsprävention (KRINKO) (2005) Infektionsprävention in Heimen. Bundesgesundheitsblatt 48(9):1061-1080

13. Kommission für Krankenhaushygiene und Infektionsprävention (KRINKO) (2002) Ausbruchmanagement und strukturiertes Vorgehen bei gehäuftem Auftreten nosokomialer Infektionen. Bundesgesundheitsblatt 45(2):180-186

14. Kommission für Krankenhaushygiene und Infektionsprävention (KRINKO) (2015) Infektionsprävention im Rahmen der Pflege und Behandlung von Patienten mit übertragbaren Krankheiten. Empfehlung der Kommission für Krankenhaushygiene und Infektionsprävention (KRINKO) beim Robert Koch-Institut. Bundesgesundheitsblatt 58(10):1151-1170

15. Kommission für Krankenhaushygiene und Infektionsprävention (KRINKO) (2016) Händehygiene in Einrichtungen des Gesundheitswesens. Bundesgesundheitsblatt 59(9):1189-1220

16. Kommission für Krankenhaushygiene und Infektionsprävention (KRINKO) (2004) Empfehlung zu den Anforderungen an die Hygiene bei der Reinigung und Desinfektion von Flächen Bundesgesundheitsblatt 4(7):51-61

17. Kommission für Krankenhaushygiene und Infektionsprävention (KRINKO) (2010) Anforderungen an die Hygiene bei der medizinischen Versorgung von immunsupprimierten Patienten. Bundesgesundheitsblatt 53(4):357-388

18. Kommission für Krankenhaushygiene und Infektionsprävention (KRINKO) (2007) Empfehlung zur Prävention nosokomialer Infektionen bei neonatologischen Intensivpflegepatienten mit einem Geburtsgewicht unter $1500 \mathrm{~g}$. Bundesgesundheitsblatt 50(10):1265-1303

19. Kommission für Krankenhaushygiene und Infektionsprävention (KRINKO) (2010) Die Kategorien in der Richtlinie für Krankenhaushygiene und Infektionsprävention - Aktualisierung der Definitionen. Mitteilung der Kommission für Krankenhaushygiene und Infektionsprävention. Bundesgesundheitsblatt 53(7):754-756

20. Magiorakos AP, Srinivasan A, Carey RB et al (2011) Multidrug-resistant, extensively drugresistant and pandrug-resistant bacteria: an international expert proposal for interim standard definitions for acquired resistance. Clin Microbiol Infect 18(3):268-281

21. Teixeira LM, Carvalho MGS, Facklam RR, Shewmaker PL (2015) Enterococcus. In: Jorgensen $\mathrm{HJ}$, Pfaller MA, Carrol KC et al (Hrsg) Manual of clinical microbiology, 11. Aufl. ASM Press, Washington, S 403-421

22. Lytsy B, Engstrand L, Gustafsson A, Kaden R (2017) Time to review the gold standard for genotyping vancomycin-resistant enterococci in epidemiology: comparing whole-genome sequencing with PFGE and MLST in three suspected outbreaks in Sweden during 2013-2015. Infect Genet Evol 54:74-80

23. Robert Koch-Institut (RKI) (2013) Surveillance nosokomialer Infektionen sowie die Erfassung von Krankheitserregern mit speziellen Resistenzen und Multiresistenzen. Fortschreibung der Liste der gemäß § 4 Abs. 2 Nr. 2 Buchstabe b in Verbindung mit $\S 23 \mathrm{Abs}$. 4 IfSG zu erfassenden nosokomialen Infektionen und Krankheitserreger mit speziellen Resistenzen und Multiresistenzen. Bundesgesundheitsblatt (56):580-583

24. Köhler W (2007) The present state of species within the genera Streptococcus and Enterococcus. Int J Med Microbiol 297(3):133-150

25. Tedim AP, Ruiz-Garbajosa P, Corander J et al (2015) Population biology of intestinal enterococcus isolates from hospitalized and nonhospitalized individuals in different age groups. Appl Environ Microbiol 81(5):1820-1831

26. Noble CJ (1978) Carriage of group D streptococci in the human bowel. J Clin Pathol 31(12):11821186

27. Fisher K, Phillips C (2009) The ecology, epidemiology and virulence of Enterococcus. Microbiology 155(Pt 6):1749-1757

28. McBride SM, Fischetti VA, Leblanc DJ, Moellering RC Jr., Gilmore MS (2007) Genetic diversity among Enterococcus faecalis. PLoS ONE 2(7): $: 582$

29. Kuch A, Willems RJ, Werner G et al (2012) Insight into antimicrobial susceptibility and population structure of contemporary human Enterococcus faecalis isolates from Europe. J Antimicrob Chemother 67(3):551-558

30. Zischka M, Kunne CT, Blom J et al (2015) Comprehensive molecular, genomic and phenotypic analysis of a major clone of Enterococcus faecalis MLST. BMC Genomics 16(175):T40

31. Leavis H, Top J, Shankar $\mathrm{N}$ et al (2004) A novel putative enterococcal pathogenicity island linked to the esp virulence gene of Enterococcus faecium and associated with epidemicity. J Bacteriol 186(3):672-682

32. Werner $G$, Fleige $C$, Geringer U, van Schaik W, Klare I, Witte W (2011) IS element IS16 as a molecular screening tool to identify hospitalassociated strains of Enterococcus faecium. BMC Infect Dis 11:80

33. Heikens $\mathrm{E}$, van Schaik W, Leavis HL, Bonten MJ, Willems RJ (2008) Identification of a novel genomic island specific to hospital-acquired clonal complex 17 Enterococcus faecium isolates. Appl Environ Microbiol 74(22):7094-7097

34. Top J, Willems R, Blok H et al (2007) Ecological replacement of Enterococcus faecalis by multiresistant clonal complex 17 Enterococcus faecium. Clin Microbiol Infect 13(3):316-319

35. Lester CH, Sandvang D, Olsen SS et al (2008) Emergence of ampicillin-resistant Enterococcus faecium in Danish hospitals. J Antimicrob Chemother 62(6):1203-1206

36. Lebreton F, van Schaik W, McGuire AM et al (2013) Emergence of epidemic multidrugresistant Enterococcus faecium from animal and commensal strains. MBio 4(4):e00534-13

37. McCracken M, Wong A, Mitchell R et al (2013) Molecular epidemiology of vancomycinresistant enterococcal bacteraemia: results from the Canadian Nosocomial Infection Surveillance Program, 1999-2009. J Antimicrob Chemother 68(7):1505-1509

38. Raven KE, Reuter S, Reynolds R et al (2016) A decade of genomic history for healthcareassociated Enterococcus faecium in the United Kingdom and Ireland. Genome Res 26(10):1388 1396

39. McAuley CM, Gobius KS, Britz ML, Craven HM (2012) Heat resistance of thermoduric enterococci isolated from milk. Int J Food Microbiol 154(3):162-168

40. Renner P, Peters J (1999) Resistance of enterococci to heat and chemical agents. Zentralbl Hyg Umweltmed 202(1):41-50

41. Kearns AM, Freeman R, Lightfoot NF (1995) Nosocomial enterococci: resistance to heat and sodium hypochlorite. J Hosp Infect 30(3):193199

42. Martinez S, Lopez M, Bernardo A (2003) Thermal inactivation of Enterococcus faecium: effect of growth temperature and physiological state of microbial cells. Lett Appl Microbiol 37(6):475481

43. Bale MJ, Bennett PM, Beringer JE, Hinton M (1993) The survival of bacteria exposed to desiccation on surfaces associated with farm buildings. J Appl Bacteriol 75(6):519-528

44. Noskin GA, Stosor V, Cooper I, Peterson LR (1995) Recovery of vancomycin-resistant enterococci on fingertips and environmental surfaces. Infect Control Hosp Epidemiol 16(10):577-581 
45. Wendt C, Wiesenthal B, Dietz E, Ruden H (1998) Survival of vancomycin-resistant and vancomycin-susceptible enterococci on dry surfaces. J Clin Microbiol 36(12):3734-3736

46. Neely AN (2000) A survey of gram-negative bacteria survival on hospital fabrics and plastics. J Burn Care Rehabil 21(6):523-527

47. Neely AN, Maley MP (2000) Survival of enterococci and staphylococci on hospital fabrics and plastic. J Clin Microbiol 38(2):724-726

48. Robine E, Derangere D, Robin D (2000) Survival of a Pseudomonas fluorescens and Enterococcus faecalis aerosol on inert surfaces. Int J Food Microbiol 55(1-3):229-234

49. Wagenvoort JH, De Brauwer El, Penders RJ, Willems RJ, Top J, Bonten MJ (2011) Environmenta survival of vancomycin-resistant Enterococcus faecium. J Hosp Infect 77(3):282-283

50. Ruiz-Garbajosa P, Bonten MJ, Robinson DA et al (2006) Multilocus sequence typing scheme for Enterococcus faecalis reveals hospital-adapted genetic complexes in a background of high rates of recombination. J Clin Microbiol 44(6):22202228

51. Zhang X, Paganelli FL, Bierschenk D et al (2012) Genome-wide identification of ampicillin resistance determinants in Enterococcus faecium. Plos Genet 8(6):e1002804

52. Galloway-Pena JR, Rice LB, Murray BE (2011) Analysis of PBP5 of early U.S. isolates of Enterococcus faecium: sequence variation alone does not explain increasing ampicillin resistance over time. Antimicrob Agents Chemother 55(7):3272-3277

53. Rice LB, Carias LL, Hutton-Thomas R, Sifaoui F, Gutmann L, Rudin SD (2001) Penicillin-binding protein 5 and expression of ampicillin resistance in Enterococcus faecium. Antimicrob Agents Chemother 45(5):1480-1486

54. Sifaoui F, Arthur M, Rice L, Gutmann L (2001) Role of penicillin-binding protein 5 in expression of ampicillin resistance and peptidoglycan structure in Enterococcus faecium. Antimicrob Agents Chemother 45(9):2594-2597

55. García-Solache M, Lebreton F, McLaughlin RE, Whiteaker JD, Gilmore MS, Rice LB (2016) Homologous recombination within large chromosomal regions facilitates acquisition of $\beta$-lactam and vancomycin resistance in Enterococcus faecium. Antimicrob Agents Chemother 60(10):5777-5786

56. Robert Koch-Institut (RKI) (2017) ARS Antibiotika-Resistenz-Surveillance. Resistenzentwicklung. https://ars.rki.de/Content/Database/ ResistanceDevelopment.aspx. Zugegriffen: 22. März 2018

57. Robert Koch-Institut (RKI), Klare I, Werner G (2015) Eigenschaften, Häufigkeit und Verbreitung von Vancomycin-resistenten Enterokokken (VRE) in Deutschland. Update 2013/2014. Epid Bull 40:439-435

58. Werner $\mathrm{G}$, Coque TM, Hammerum AM et al (2008) Emergence and spread of vancomycin resistance among enterococci in Europe. Euro Surveill 13(47):pii: 19046

59. Willems RJ, van Schaik W (2009) Transition of Enterococcus faecium from commensal organism to nosocomial pathogen. Future Microbiol 4(9):1125-1135

60. European Centre for Disease Prevention and Control (ECDC) (2016) Data from the ECDC Surveillance Atlas - antimicrobial resistance. https:// ecdc.europa.eu/en/antimicrobial-resistance/ surveillance-and-disease-data/data-ecdc. Zugegriffen: 22. März 2018

61. Hodel-Christian SL, Murray BE (1991) Characterization of the gentamicin resistance transposon Tn5281 from Enterococcus faecalis and comparison to staphylococcal transposons $\operatorname{Tn} 4001$ and Tn4031. Antimicrob Agents Chemother 35(6):1147-1152

62. Naser SM, Vancanneyt M, Hoste B, Snauwaert C, Vandemeulebroecke K, Swings J (2006) Reclassification of Enterococcus flavescens Pompei et al. 1992 as a later synonym of Enterococcus casseliflavus (ex Vaughan et al. 1979) Collins et al. 1984 and Enterococcus saccharominimus Vancanneyt et al. 2004 as a later synonym of Enterococcus italicus Fortina et al. 2004. Int J Syst Evol Microbiol 56(Pt 2):413-416

63. Freitas AR, Tedim AP, Francia MV et al (2016) Multilevel population genetic analysis of vanA and vanB Enterococcus faecium causing nosocomial outbreaks in 27 countries (1986-2012). J Antimicrob Chemother 71(12):3351-3366

64. Conlan S, Thomas PJ, Deming C et al (2014) Single-molecule sequencing to track plasmid diversity of hospital-associated carbapenemaseproducing Enterobacteriaceae. Sci Transl Med 6(254):254ra126

65. Sivertsen A, Billstrom $\mathrm{H}$, Melefors $\mathrm{O}$ et al (2014) A multicentre hospital outbreak in Sweden caused by introduction of a vanB2 transposon into a stably maintained pRUM-plasmid in an Enterococcus faecium ST192 clone. PLoS ONE 9(8):e103274

66. Holmes NE, Ballard SA, Lam MM et al (2013) Genomic analysis of teicoplanin resistance emerging during treatment of vanB vancomycin-resistant Enterococcus faecium infections in solid organ transplant recipients including donor-derived cases. J Antimicrob Chemother 68(9):2134-2139

67. Werner G, Fleige C, Ewert B, Laverde-Gomez JA, Klare I, Witte W (2010) High-level ciprofloxacin resistance among hospital-adapted Enterococcus faecium (CC17). Int J Antimicrob Agents 35(2):119-125

68. Willems RJ, Top J, van Schaik W et al (2012) Restricted gene flow among hospital subpopulations of Enterococcus faecium. MBio 3(4):e00151 12

69. Willems RJ, Homan W, Top J et al (2001) Variant esp gene as a marker of a distinct genetic lineage of vancomycin-resistant Enterococcus faecium spreading in hospitals. Lancet 357(9259):853-855

70. Rice LB, Carias L, Rudin S et al (2003) A potential virulence gene, hylEfm, predominates in Enterococcus faecium of clinical origin. J Infect Dis 187(3):508-512

71. Raven KE, Gouliouris T, Brodrick H et al (2017) Complex routes of nosocomial Vancomycinresistant Enterococcus faecium transmission revealed by genome sequencing. Clin Infect Dis 64(7):886-893

72. Bender JK, Kalmbach A, Fleige C, Klare I, Fuchs S, Werner G (2016) Population structure and ac quisition of the vanB resistance determinant in German clinical isolates of Enterococcus faecium ST192. Sci Rep 6:21847

73. Klare I, Konstabel C, Mueller-Bertling $S$ et al (2005) Spread of ampicillin/vancomycin-resistant Enterococcus faecium of the epidemic-virulent clonal complex-17 carrying the genes esp and hyl in German hospitals. Eur J Clin Microbiol Infect Dis 24(12):815

74. Ballard SA, Pertile KK, Lim M, Johnson PD, Grayson ML (2005) Molecular characterization of vanB elements in naturally occurring gut anaerobes. Antimicrob Agents Chemother 49(5):1688-1694

75. Graham M, Ballard SA, Grabsch EA, Johnson PD, Grayson ML (2008) High rates of fecal carriage of nonenterococcal vanB in both children and adults. Antimicrob Agents Chemother 52(3):1195-1197

76. Klare I, Fleige C, Geringer U, Witte W, Werner G (2012) Performance of three chromogenic VRE screening agars, two Etest ${ }^{\oplus}$ vancomycin protocols, and different microdilution methods in detecting vanB genotype Enterococcus faecium with varying vancomycin MICs. Diagn Microbiol Infect Dis 74(2):171-176

77. European Centre for Disease Prevention and Control (ECDC) (2017) Annual surveillance reports on antimicrobial resistance. https://ecdc europa.eu/en/antimicrobial-resistance/surveillance-and-disease-data/report. Zugegriffen: 22. März 2018

78. Robert Koch-Institut (RKI) (2017) ARS - Antibiotika-Resistenz-Surveillance. https://ars.rki.de/. Zugegriffen: 22. März 2018

79. Paul-Ehrlich-Gesellschaft für Chemotherapie e.V.(PEG), Arbeitsgemeinschaft Empfindlichkeitsprüfungen und Resistenz (2017) Resistenzdaten. http://www.p-e-g.org/econtext/ resistenzdaten. Zugegriffen: 22. März 2018

80. Surveillance der Antibiotika-Anwendung und bakteriellen Resistenzen auf Intensivstationen (SARI) (2018) SARI - Resistenzdaten. http://sari. eu-burden.info/rr.php. Zugegriffen: 22. März 2018

81. Noll I, Schweickert B, Abu SM, Feig M, Claus H, Eckmanns T (2012) Daten zur Antibiotikaresistenzlage in Deutschland. Vier Jahre AntibiotikaResistenz-Surveillance (ARS). Bundesgesundheitsblatt 55(11-12):1370-1376

82. Werner G, Klare I, Fleige C et al (2012) Vancomycin-resistant vanB-type Enterococcus faecium isolates expressing varying levels of vancomycin resistance and being highly prevalent among neonatal patients in a single ICU. Antimicrob Resist Infect Control 1(1):21

83. Whelton E, Lynch C, O'Reilly B et al (2016) Vancomycin-resistant enterococci carriage in an acute Irish hospital. J Hosp Infect 93(2):175-180

84. de Niederhäusern S, Bondi M, Messi P et al (2011) Vancomycin-resistance transferability from VanA enterococci to Staphylococcus aureus. Curr Microbiol 62(5):1363-1367

85. Centers for Disease Control and Prevention (CDC) (2002) Staphylococcus aureus resistant to vancomycin - United States, 2002. MMWR Morb Mortal Wkly Rep 51(26):565-567

86. Walters MS, Eggers P, Albrecht $\mathrm{V}$ et al (2015) Notes from the Field: Vancomycin-Resistant Staphylococcus aureus - Delaware, 2015. Mmwr Morb Mortal Wkly Rep 64(37):1056

87. Faron ML, Ledeboer NA, Buchan BW (2016) Resistance mechanisms, epidemiology, and approaches to screening for Vancomycin-resistant Enterococcus in the health care setting. J Clin Microbiol 54(10):2436-2447

88. Melo-Cristino J, Resina C, Manuel V, Lito L, Ramirez M (2013) First case of infection with vancomycin-resistant Staphylococcus aureus in Europe. Lancet 382(9888):205 
89. Norrby R (2001) Linezolid - a review of the first oxazolidinone. Expert Opin Pharmacother 2(2):293-302

90. Burdette SD, Trotman R (2015) Tedizolid: the first once-daily Oxazolidinone class antibiotic. Clin Infect Dis 61(8):1315-1321

91. Tewhey R, Gu B, Kelesidis T et al (2014) Mechanisms of linezolid resistance among coagulasenegative staphylococci determined by wholegenome sequencing. MBio 5(3):e894-14

92. Gu B, Kelesidis T, Tsiodras S, Hindler J, Humphries RM (2013) The emerging problem of linezolid-resistant Staphylococcus. J Antimicrob Chemother 68(1):4-11

93. Bender J, Strommenger B, Steglich M et al (2015) Linezolid resistance in clinical isolates of staphylococcus epidermidis from German hospitals and characterization of two cfr-carrying plasmids. J Antimicrob Chemother 70(6):1630-1638

94. Cuny C, Arnold P, Hermes J et al (2017) Occurrence of cfr-mediated multiresistance in staphylococci from veal calves and pigs, from humans at the corresponding farms, and from veterinarians and their family members. Vet Microbiol 200:88-94

95. Liu Y, Wang Y, Schwarz S et al (2014) Investigation of a multiresistance gene cfr that fails to mediate resistance to phenicols and oxazolidinones in Enterococcus faecalis. J Antimicrob Chemother 69(4):892-898

96. Bender JK, Fleige C, Klare I et al (2016) Detection of a $\mathrm{cfr}(\mathrm{B})$ variant in German Enterococcus faecium clinical isolates and the impact on Linezolid resistance in Enterococcus spp. PLoS ONE 11(11):e167042

97. Klare I, Fleige C, Geringer U et al (2015) Increased frequency of linezolid resistance among clinical Enterococcus faecium isolates from German hospital patients. J Glob Antimicrob Resist 3(2):128-131

98. Rubinstein E, Vaughan D (2005) Tigecycline: a novel glycylcycline. Drugs 65(10):1317-1336

99. Frampton JE, Curran MP (2005) Tigecycline. Drugs 65(18):2623-2635

100. Entenza JM, Moreillon P (2009) Tigecycline in combination with other antimicrobials: a review of in vitro, animal and case report studies. Int J Antimicrob Agents 34(1):8.e1-8.e9

101. Jenkins I (2007) Linezolid- and vancomycinresistant Enterococcus faecium endocarditis: successful treatment with tigecycline and daptomycin. J Hosp Med 2(5):343-344

102. Polidori M, Nuccorini $A$, Tascini $C$ et al (2011) Vancomycin-resistant Enterococcus faecium (VRE) bacteremia in infective endocarditis successfully treated with combination daptomycin and tigecycline. J Chemother 23(4):240-241

103. Jaspan HB, Brothers AW, Campbell AJ et al (2010) Multidrug-resistant Enterococcus faecium meningitis in a toddler: characterization of the organism and successful treatment with intraventricular daptomycin and intravenous tigecycline. Pediatr Infect Dis J 29(4):379-381

104. Shen $F$, Han $Q$, Xie D, Fang $M$, Zeng $H$, Deng $Y(2015)$ Efficacy and safety of tigecycline for the treatment of severe infectious diseases: an updated meta-analysis of RCTs. Int J Infect Dis 39:25-33

105. Fiedler S, Bender JK, Klare I et al (2016) Tigecycline resistance in clinical isolates of Enterococcus faecium is mediated by an upregulation of plasmid-encoded tetracycline determinants tet( $\mathrm{L})$ and tet(M). J Antimicrob Chemother 71(4):871-881

106. Niebel M, Quick J, Prieto AM et al (2015) Deletions in a ribosomal protein-coding gene are associated with tigecycline resistance in Enterococcus faecium. Int J Antimicrob Agents 46(5):572-575

107. Werner G, Gfrorer S, Fleige C, Witte W, Klare I (2008) Tigecycline-resistant Enterococcus faecalis strain isolated from a German intensive care unit patient. J Antimicrob Chemother 61(5):1182-1183

108. Lee SY, Fan HW, Kuti JL, Nicolau DP (2006) Update on daptomycin: the first approved lipopeptide antibiotic. Expert Opin Pharmacother 7(10):1381-1397

109. The European Committee on Antimicrobial Susceptibility Testing (EUCAST) (2016) Guidance document on use of daptomycin to treat enterococcal endocarditis. http://www.eucast. org/fileadmin/src/media/PDFs/EUCAST_files/ General_documents/EUCAST_daptomycin_ guidance_note_20160302.pdf. Zugegriffen: 22. März 2018

110. Ceron I, Munoz P, Marin M et al (2014) Efficacy of daptomycin in the treatment of enterococcal endocarditis: a 5 year comparison with conventional therapy. J Antimicrob Chemother 69(6):1669-1674

111. Shukla BS, Shelburne S, Reyes K et al (2016) Influence of minimum inhibitory concentration in clinical outcomes of Enterococcus faecium Bacteremia treated with Daptomycin: is it time to change the breakpoint? Clin Infect Dis 62(12):1514-1520

112. Snyder AH, Werth BJ, Barber KE, Sakoulas G, Rybak MJ (2014) Evaluation of the novel combination of daptomycin plus ceftriaxone against vancomycin-resistant enterococci in an in vitro pharmacokinetic/pharmacodynamic simulated endocardial vegetation model. J Antimicrob Chemother 69(8):2148-2154

113. Hindler JA, Wong-Beringer $A$, Charlton $C L$ et al (2015) In vitro activity of daptomycin in combination with beta-lactams, gentamicin, rifampin, and tigecycline against daptomycinnonsusceptible enterococci. Antimicrob Agents Chemother 59(7):4279-4288

114. Desai H, Wong R, Pasha AK (2016) A novel way of treating multidrug-resistant Enterococci. $\mathrm{N} \mathrm{Am} \mathrm{J}$ Med Sci 8(5):229-231

115. Chuang YC, Chen PY, Lin CY, Chen YC, Wang JT, Chang SC (2018) A retrospective clinical comparison of daptomycin vs daptomycin and a beta-lactam antibiotic for treating vancomycinresistant Enterococcus faecium bloodstream infections. Sci Rep 8(1):1632

116. Tran TT, Munita JM, Arias CA (2015) Mechanisms of drug resistance: daptomycin resistance. Ann N Y Acad Sci 1354:32-53

117. Werth BJ, Steed ME, Ireland CE et al (2014) Defining daptomycin resistance prevention exposures in vancomycin-resistant Enterococcus faecium and E. faecalis. Antimicrob Agents Chemother 58(9):5253-5261

118. Tran TT, Panesso D, Gao H et al (2013) Wholegenome analysis of a daptomycin-susceptible Enterococcus faecium strain and its daptomycinresistant variant arising during therapy. Antimicrob Agents Chemother 57(1):261-268

119. Arias CA, Panesso D, McGrath DM et al (2011) Genetic basis for in vivo daptomycin resistance in enterococci. N Engl J Med 365(10):892-900
120. Lellek H, Franke GC, Ruckert C et al (2015) Emergence of daptomycin non-susceptibility in colonizing vancomycin-resistant Enterococcus faecium isolates during daptomycin therapy. Int J Med Microbiol 305(8):902-909

121. Donskey CJ, Chowdhry TK, Hecker MT et al (2000) Effect of antibiotic therapy on the density of vancomycin-resistant enterococci in the stool of colonized patients. N Engl J Med 343(26):1925-1932

122. Ubeda C, Taur Y, Jenq RR et al (2010) Vancomycin-resistant Enterococcus domination of intestinal microbiota is enabled by antibiotic treatment in mice and precedes bloodstream invasion in humans. J Clin Invest 120(12):43324341

123. Taur Y, Xavier JB, Lipuma L et al (2012) Intestinal domination and the risk of bacteremia in patients undergoing allogeneic hematopoietic stem cell transplantation. Clin Infect Dis 55(7):905914

124. Buffie CG, Pamer EG (2013) Microbiota-mediated colonization resistance against intestinal pathogens. Nat Rev Immunol 13(11):790-801

125. Hooton TM, Roberts PL, Cox ME, Stapleton AE (2013) Voided midstream urine culture and acute cystitis in premenopausal women. N Engl J Med 369(20):1883-1891

126. Ziakas P, Pliakos E, Zervou F, Knoll B, Rice L, Mylonakis E (2014) MRSA and VRE colonization in solid organ transplantation: a meta-analysis of published studies. Am J Transplant 14(8):18871894

127. Ong DS, Bonten MJ, Safdari K et al (2015) Epidemiology, management, and risk-adjusted mortality of ICU-acquired Enterococcal Bacteremia. Clin Infect Dis 61(9):1413-1420

128. Dupont H, Friggeri A, Touzeau J et al (2011) Enterococci increase the morbidity and mortality associated with severe intra-abdominal infections in elderly patients hospitalized in the intensive care unit. J Antimicrob Chemother 66:2379-2385

129. Kaffarnik MF, Urban M, Hopt UT, Utzolino S (2012) Impact of enterococcus on immunocompetent and immunosuppressed patients with perforation of the small or large bowel. Technol Health Care 20(1):37-48

130. Jindai K, Strerath M, Hess T, Safdar N (2014) Is a single positive blood culture for Enterococcus species representative of infection or contamination? Eur J Clin Microbiol Infect Dis 33(11):1995-2003

131. Grundmann H, Bärwolff S, Tami A et al (2005) How many infections are caused by patient-topatient transmission in intensive care units? Crit Care Med 33(5):946-951

132. Kola A, Schwab F, Bärwolff S et al (2010) Is there an association between nosocomial infection rates and bacterial cross transmissions? Crit Care Med 38(1):46-50

133. Erb S, Frei R, Dangel M, Widmer AF (2017) Multidrug-resistant organisms detected more than 48 hours after hospital admission are not necessarily hospital-acquired. Infect Control Hosp Epidemiol 38(1):18-23

134. Cheng VC, Tai JW, Ng ML et al (2012) Extensive contact tracing and screening to control the spread of vancomycin-resistant Enterococcus faecium ST414 in Hong Kong. Chin Med J 125(19):3450-3457

135. Kurup A, Chlebicki M, Ling M et al (2008) Control of a hospital-wide vancomycin-resistant 
Enterococci outbreak. Am J Infect Control 36(3):206-211

136. Kaki R, Yu Y, O’Neill C, Lee C, Mertz D (2014) Vancomycin-resistant enterococcus (VRE) transmission and risk factors in contacts of VRE carriers. Infect Control Hosp Epidemiol 35(07):876-879

137. Zhou Q, Moore C, Eden S, Tong A, McGeer A (2008) Factors associated with acquisition of vancomycin-resistant enterococci (VRE) in roommate contacts of patients colonized or infected with VRE in a tertiary care hospital. Infect Control Hosp Epidemiol 29(05):398-403

138. Austin DJ, Bonten MJ (1998) Vancomycinresistant enterococci in intensive care hospital settings. Mem Inst Oswaldo Cruz 93(5):587-588

139. Duckro AN, Blom DW, Lyle EA, Weinstein RA, Hayden MK (2005) Transfer of vancomycin-resistant enterococci via health care worker hands. Arch Intern Med 165(3):302-307

140. Bonten MJ, Hayden MK, Nathan C et al (1996) Epidemiology of colonisation of patients and environment with vancomycin-resistant enterococci. Lancet 348(9042):1615-1619

141. Gray J, George R (2000) Experience of vancomycin-resistant enterococci in a children's hospital. J Hosp Infect 45(1):11-18

142. McCarthy K, Van Nierop W, Duse A et al (2000) Control of an outbreak of vancomycin-resistant Enterococcus faecium in an oncology ward in South Africa: effective use of limited resources. J Hosp Infect 44(4):294-300

143. Hanna H, Umphrey J, Tarrand J, Mendoza M, Raad I (2001) Management of an outbreak of vancomycin-resistant enterococci in the medical intensive care unit of a cancer center. Infect Control Hosp Epidemiol 22(04):217-219

144. Martinez JA, Ruthazer R, Hansjosten K, Barefoot L, Snydman DR (2003) Role of environmental contamination as a risk factor for acquisition of vancomycin-resistant enterococci in patients treated in a medical intensive care unit. Arch Intern Med 163(16):1905-1912

145. Lemmen S, Häfner H, Zolldann D, Stanzel S, Lütticken R (2004) Distribution of multi-resistant Gram-negative versus Gram-positive bacteria in the hospital inanimate environment. J Hosp Infect 56(3):191-197

146. Rhinehart E, Smith NE, Wennersten C et al (1990) Rapid dissemination of $\beta$-Lactamase-producing, Aminoglycoside-resistant Enterococcus faecalis among patients and staff on an infant-toddler surgical ward. N Engl J Med 323(26):1814-1818

147. Boyce JM, Opal SM, Chow JW et al (1994) Outbreak of multidrug-resistant Enterococcus faecium with transferable vanB class vancomycin resistance. J Clin Microbiol 32(5):1148-1153

148. Schulz-Stübner S, Schmidt-Warnecke A, Hwang JH (2013) VRE transmission via the reusable breathing circuit of a transport ventilator: outbreak analysis and experimental study of surface disinfection. Intensive Care Med 39(5):975-976

149. Golan Y, Doron S, Sullivan B, Snydman DR (2005) Transmission of vancomycin-resistant enterococcus in a neonatal intensive care unit. Pediatr Infect Dis J 24(6):566-567

150. Porwancher R, Sheth A, Remphrey S, Taylor E, Hinkle C, Zervos M (1997) Epidemiological study of hospital-acquired infection with vancomycin-resistant Enterococcus faecium: possible transmission by an electronic ear-probe thermometer. Infect Control Hosp Epidemiol 18(11):771-773
151. Livornese LL Jr., Dias S, Samel C et al (1992) Hospital-acquired infection with vancomycinresistant Enterococcus faecium transmitted by electronic thermometers. Ann Intern Med 117(2):112-116

152. Lee SC, Wu MS, Shih HJ et al (2013) Identification of vancomycin-resistant enterococci clones and inter-hospital spread during an outbreak in Taiwan. BMC Infect Dis 13:163

153. Falk PS, Winnike J, Woodmansee C, Desai M, Mayhall CG (2000) Outbreak of vancomycin-resistant enterococci in a burn unit. Infect Control Hosp Epidemiol 21(9):575-582

154. Huang SS, Datta R, Platt R (2006) Risk of acquiring antibiotic-resistant bacteria from prior room occupants. Arch Intern Med 166(18):1945-1951

155. Drees M, Snydman DR, Schmid CH et al (2008) Prior environmental contamination increases the risk of acquisition of vancomycin-resistant enterococci. Clin Infect Dis 46(5):678-685

156. Noble MA, Isaac-Renton JL, Bryce EA et al (1998) The toilet as a transmission vector of vancomycin-resistant enterococci. J Hosp Infect 40(3):237-241

157. Wendt C, Ruden H, Edmond M (1998) Vancomycin-resistente Enterokokken Epidemiologie, Risikofaktoren und Pravention. Dtsch Arztebl 95(25):A-1604-A1610

158. Gerba CP, Wuollet AL, Raisanen P, Lopez GU (2016) Bacterial contamination of computer touch screens. Am J Infect Control 44(3):358360

159. Schultz M, Gill J, Zubairi S, Huber R, Gordin F (2003) Bacterial contamination of computer keyboards in a teaching hospital. Infect Control Hosp Epidemiol 24(4):302-303

160. Koroglu M, Gunal S, Yildiz F, Savas M, Ozer A, Altindis M (2015) Comparison of keypads and touch-screen mobile phones/devices as potential risk for microbial contamination. J Infect Dev Ctries 9(12):1308-1314

161. Kandel CE, Simor AE, Redelmeier DA (2014) Elevator buttons as unrecognized sources of bacterial colonization in hospitals. Open Med 8(3):e81-86

162. Hayden MK, Blom DW, Lyle EA, Moore CG, Weinstein RA (2008) Risk of hand or glove contamination after contact with patients colonized with vancomycin-resistant enterococcus or the colonized patients' environment. Infect Control Hosp Epidemiol 29(2):149-154

163. Morgan DJ, Rogawski E, Thom KA et al (2012) Transfer of multidrug-resistant bacteria to healthcare workers' gloves and gowns after patient contact increases with environmental contamination. Crit Care Med 40(4):1045

164. Whitman MS, Pitsakis PG, DeJesus E, Osborne AJ, Levison ME, Johnson CC (1996) Gastrointestinal tract colonization with vancomycin-resistant Enterococcus faecium in an animal model. Antimicrob Agents Chemother 40(6):1526-1530

165. Dancer SJ (2014) Controlling hospital-acquired infection: focus on the role of the environment and new technologies for decontamination. Clin Microbiol Rev 27(4):665-690

166. Monteserin N, Larson E (2016) Temporal trends and risk factors for healthcare-associated vancomycin-resistant enterococci in adults. J Hosp Infect 94(3):236-241

167. Humphreys H (2014) Controlling the spread of vancomycin-resistant enterococci. Is active screening worthwhile? J Hosp Infect 88(4):191198
168. Cohen MJ, Adler A, Block C et al (2009) Acquisition of vancomycin-resistant enterococci in internal medicine wards. Am J Infect Control 37(2):111-116

169. Rabinowitz RP, Kufera JA, Makley MJ (2012) A hidden reservoir of methicillin-resistant Staphylococcus aureus and vancomycin-resistant Enterococcus in patients newly admitted to an acute rehabilitation hospital. PM R 4(1):18-22

170. Carmeli Y, Eliopoulos GM, Samore MH (2002) Antecedent treatment with different antibiotic agents as a risk factor for vancomycin-resistant Enterococcus. Emerging Infect Dis 8(8):802-807

171. Paterson DL, Muto CA, Ndirangu M et al (2008) Acquisition of rectal colonization by vancomycin-resistant Enterococcus among intensive care unit patients treated with piperacillin-tazobactam versus those receiving cefepime-containing antibiotic regimens. Antimicrob Agents Chemother 52(2):465-469

172. Hayakawa K, Marchaim D, Palla M et al (2013) Epidemiology of vancomycin-resistant Enterococcus faecalis: a case-case-control study. Antimicrob Agents Chemother 57(1):49-55

173. Ajao AO, Harris AD, Roghmann M-C et al (2011) Systematic review of measurement and adjustment for colonization pressure in studies of methicillin-resistant Staphylococcus aureus, vancomycin-resistant enterococci, and Clostridium difficile acquisition. Infect Control Hosp Epidemiol 32(5):481

174. Tacconelli E, Karchmer AW, Yokoe D, D'agata EM (2004) Preventing the influx of vancomycin-resistant enterococci into health care institutions, by use of a simple validated prediction rule. Clin Infect Dis 39(7):964-970

175. Decker BK, Lau AF, Dekker JP et al (2017) Healthcare personnelintestinal colonization with multidrug-resistant organisms. Clin Microbiol Infect 24(1):82.e1-82.e4

176. Baran J Jr., Ramanathan J, Riederer KM, Khatib $R$ (2002) Stool colonization with vancomycinresistant enterococci in healthcare workers and their households. Infect Control Hosp Epidemiol 23(1):23-26

177. Montecalvo MA, de Lencastre $\mathrm{H}$, Carraher $\mathrm{M}$ et al (1995) Natural history of colonization with vancomycin-resistant Enterococcus faecium. Infect Control Hosp Epidemiol 16(12):680-685

178. Kim AS (2011) Using the good to beat out the bad: probiotics for eliminating vancomycinresistant enterococci colonization. J Clin Gastroenterol 45(10):844-845

179. Baden L, Thiemke W, Skolnik A et al (2001) Prolonged colonization with vancomycin-resistant Enterococcus faecium in long-term care patients and the significance of "clearance". Clin Infect Dis 33(10):1654-1660

180. Shenoy ES, Paras ML, Noubary F, Walensky RP, Hooper DC (2014) Natural history of colonization with methicillin-resistant Staphylococcus aureus (MRSA) and vancomycin-resistant Enterococcus (VRE): a systematic review. BMC Infect Dis 14(1):177

181. Sohn KM, Peck KR, Joo EJ et al (2013) Duration of colonization and risk factors for prolonged carriage of vancomycin-resistant enterococci after discharge from the hospital. Int J Infect Dis 17(4):e240-246

182. Karki S, Land G, Aitchison S et al (2013) Long-term carriage of vancomycin-resistant enterococci in patients discharged from hospi- 
tals: a 12-year retrospective cohort study. J Clin Microbiol 51(10):3374-3379

183. Nourse C, Byrne C, Murphy H, Kaufmann M, Clarke A, Butler K (2000) Eradication of vancomycin resistant Enterococcus faecium from a paediatric oncology unit and prevalence of colonization in hospitalized and community-based children. Epidemiol Infect 124(01):53-59

184. Henning KJ, Delencastre H, Eagan J et al (1996) Vancomycin-resistant Enterococcus faecium on a pediatric oncology ward: duration of stool shedding and incidence of clinical infection. Pediatr Infect Dis J 15(10):848-854

185. Schuster F, Graubner UB, Schmid I, Weiss M, Belohradsky BH (1998) Vancomycin-resistant-enterococci-colonization of 24 patients on a pediatric oncology unit. Klin Padiatr 210(04):261-263

186. Hopkins HA, Sinkowitz-Cochran RL, Rudin BA, Keyserling HL, Jarvis WR (2000) Vancomycin use in pediatric hematology-oncology patients. Infect Control Hosp Epidemiol 21(01):48-50

187. Lister DM, Tan K, Carse E, Stuart RL (2016) Clearance of infant vancomycin-resistant Enterococcus faecium carriage after a neonatal inpatient outbreak. Am J Infect Control 44(10):1172-1173

188. Ghosh A, Jiao L, Al-Mutawa F et al (2014) Value of an active surveillance policy to document clearance of meticillin-resistant Staphylococcus aureus and vancomycin-resistant enterococci amongst inpatients with prolonged admissions. J Hosp Infect 88(4):230-233

189. Behnke M, Hansen S, Leistner R et al (2013) Nosocomial infection and antibiotic use. Dtsch Arztebl Int 110:627-633

190. Nationales Referenzzentrum für die Surveillance von nosokomialen Infektionen (NRZ) (2017) Deutsche nationale Punkt-Prävalenzerhebung zu nosokomialen Infektionen und Antibiotika-Anwendung. 2016. Abschlussbericht. http://www.nrz-hygiene.de/fileadmin/nrz/ download/pps2016/PPS_2016_Abschlussbericht_20.07.2017.pdf. Zugegriffen: 22. März 2018

191. Orsi G, Ciorba V (2013) Vancomycin resistant enterococci healthcare associated infections. Ann Ig 25(6):485-492

192. Gudiol C, Ayats J, Camoez M et al (2013) Increase in bloodstream infection due to vancomycinsusceptible Enterococcus faecium in cancer patients: risk factors, molecular epidemiology and outcomes. PLoS ONE 8(9):e74734

193. Kresken M, Groll AH, Lass-Flörl C, Körber-Irrgang B (2013) PEG-Resistenzstudie. Epidemiologie und Resistenzsituation bei Candida-Isolaten aus Blut und anderen primär sterilen Körperregionen gegenüber Antimykotika. Bericht über die Ergebnisse einer multizentrischen Studie der Arbeitsgemeinschaft Empfindlichkeitsprüfungen \& Resistenz der Paul-Ehrlich-Gesellschaft für Chemotherapie e.V. aus dem Jahre 2010. Antiinfectives Intelligence: Rheinbach. http:// www.paul-ehrlich-gesellschaft.de/econtext/ Poster\%20Publikationen. Zugegriffen: 22. März 2018

194. Kresken M, Hafner D (1999) Prävalenz der Antibiotikaresistenz bei klinisch wichtigen Infektionserregern in Mitteleuropa. Bericht über die Ergebnisse einer multizentrischen Studie der Arbeitsgemeinschaft "Resistenz" in der PaulEhrlich-Gesellschaft für Chemotherapie e.V. aus dem Jahre 1998. http://www.p-e-g.org/econtext/Berichte\%20der\%20Studien. Zugegriffen: 22 März 2018
195. Kresken $M$, Hafner D, Körber-Irrgang $B$ für die Studiengruppe (2013) PEG-Resistenzstudie. Epidemiologie und Resistenzsituation bei klinisch wichtigen Infektionserregern aus dem Hospitalbereich gegenüber Antibiotika. Bericht über die Ergebnisse einer multizentrischen Studie der Arbeitsgemeinschaft Empfindlichkeitsprüfungen \& Resistenz der Paul-Ehrlich-Gesellschaft für Chemotherapie e.V. aus dem Jahre 2010. Antiinfectives Intelligence: Rheinbach. http:// www.p-e-g.org/econtext/Berichte\%20der\%20 Studien. Zugegriffen: 22. März 2018

196. Kresken $M$, Hafner $D$, Körber-Irrgang $B$ für die Studiengruppe (2013) PEG-Resistenzstudie. Resistenzsituation bei klinisch wichtigen Infektionserregern aus dem ambulanten Versorgungsbereich gegenüber Antibiotika. Bericht über die Ergebnisse einer multizentrischen Studie der Arbeitsgemeinschaft Empfindlichkeitsprüfungen \& Resistenz der Paul-Ehrlich-Gesellschaft für Chemotherapie e.V. aus dem Jahre 2010. Antiinfectives Intelligence: Rheinbach. http:// www.p-e-g.org/econtext/Berichte\%20der\%20 Studien. Zugegriffen: 22. März 2018

197. Kresken $M$, Hafner D, Körber-Irrgang $B$ für die Studiengruppe (2016) PEG-Resistenzstudie. Epidemiologie und Resistenzsituation bei klinisch wichtigen Infektionserregern aus dem Hospitalbereich gegenüber Antibiotika. Bericht über die Ergebnisse einer multizentrischen Studie de Arbeitsgemeinschaft Empfindlichkeitsprüfungen \& Resistenz der Paul-Ehrlich-Gesellschaft für Chemotherapie e.V. aus dem Jahre 2013. Antiinfectives Intelligence: Rheinbach. http:// www.p-e-g.org/econtext/Berichte\%20der\%20 Studien. Zugegriffen: 22. März 2018

198. Kresken $M$, Hafner D, Körber-Irrgang $B$ für die Studiengruppe (2016) PEG-Resistenzstudie. Epidemiologie und Resistenzsituation bei klinisch wichtigen Infektionserregern aus dem ambulanten Versorgungsbereich gegenüber Antibiotika. Bericht über die Ergebnisse einer multizentrischen Studie der Arbeitsgemeinschaft Empfindlichkeitsprüfungen \& Resistenz der Paul-Ehrlich-Gesellschaft für Chemotherapie e.V. aus dem Jahre 2013. Antiinfectives Intelligence: Rheinbach. http://www.p-e-g.org/econtext/Berichte\%20der\%20Studien. Zugegriffen: 22. März 2018

199. Kresken M, Hafner D, Schmitz FJ, Wichelhaus TA für die Studiengruppe (2003) PEG-Resistenzstudie. Resistenzsituation bei klinisch wichtigen Infektionserregern gegenüber Antibiotika in Deutschland und im mitteleuropäischen Raum. Bericht über die Ergebnisse einer multizentrischen Studie der Arbeitsgemeinschaft Empfindlichkeitsprüfungen \& Resistenz der Paul-EhrlichGesellschaft für Chemotherapie e.V. aus dem Jahre 2001. Antiinfectives Intelligence: Bonn. http://www.p-e-g.org/econtext/Berichte\%20 der\%20Studien. Zugegriffen: 22. März 2018

200. Kresken $M$, Hafner D, Schmitz F-J, Wichelhaus TA für die Studiengruppe (2006) PEG-Resistenzstudie. Resistenzsituation bei klinisch wichtigen Infektionserregern gegenüber Antibiotika in Deutschland und im mitteleuropäischen Raum. Bericht über die Ergebnisse einer multizentrischen Studie der Arbeitsgemeinschaft Empfindlichkeitsprüfungen \& Resistenz der Paul-Ehrlich-Gesellschaft für Chemotherapie e.V. aus dem Jahre 2004. Antiinfectives Intelligence: Rheinbach. http://www.p-e-g.org/econtext/Berichte\%20der\%20Studien. Zugegriffen: 22. März 2018
201. Kresken M, Hafner D, Schmitz F-J, Wichelhaus TA für die Studiengruppe (2009) PEG-Resistenzstudie. Resistenzsituation bei klinisch wichtigen Infektionserregern gegenüber Antibiotika in Deutschland und im mitteleuropäischen Raum. Bericht über die Ergebnisse einer multizentrischen Studie der Arbeitsgemeinschaft Empfindlichkeitsprüfungen \& Resistenz der Paul-Ehrlich-Gesellschaft für Chemotherapie e.V. aus dem Jahre 2007. Antiinfectives Intelligence: Rheinbach. http://www.p-e-g.org/econtext/Berichte\%20der\%20Studien. Zugegriffen: 22. März 2018

202. Nationales Referenzzentrum für Surveillance von nosokomialen Infektionen (2017) KISS Krankenhaus-Infektions-Surveillance-System. Modul OP-KISS. Referenzdaten. Berechnungszeitraum: Januar 2012 bis Dezember 2016. http:// www.nrz-hygiene.de/fileadmin/nrz/module/ op/201201_201612_OPRef.pdf. Zugegriffen: 22 . März 2018

203. Pochhammer J, Kramer A, Schaffer M (2017) Enterokokken und postoperative Wundinfektionen. Verursacher oder harmloser Kommensale? Chirurg 88(5):377-384

204. Nationales Referenzzentrum für Surveillance von nosokomialen Infektionen (2017) KISS Krankenhaus-Infektions-Surveillance-System. Infektionssurveillance im Modul ITS-KISS. Referenzdaten. Berechnungszeitraum: Januar 2012 bis Dezember 2016. http://www.nrz-hygiene. de/fileadmin/nrz/module/its/201201_201612_ ALLE_ITSRef.pdf. Zugegriffen: 22. März 2018

205. Heyde A (2014) Befunde, prädisponierende Faktoren und klinische Verläufe der infektiösen Endokarditis unter Berücksichtigung des Lebensalters. Dissertation, Medizinischen Fakultät Charité - Universitätsmedizin Berlin

206. Guerrero MLF, Goyenechea A, Verdejo C, Roblas RF, de Górgolas M (2007) Enterococcal endocarditis on native and prosthetic valves: a review of clinical and prognostic factors with emphasis on hospital-acquired infections as a major determinant of outcome. Medicine (Baltimore) 86(6):363-377

207. Bandyk DF, Novotney ML, Back MR, Johnson BL, Schmacht DC (2001) Expanded application of in situ replacement for prosthetic graft infection. J Vasc Surg 34(3):411-420

208. Yogev R (2001) Antibiotic therapy of an enterococcal ventriculoperitoneal shunt infection. Pediatr Infect Dis J 20(8):816-817

209. Barequet IS, Sullivan Baker A, Schein OE (2000) Ocular infections. In: Waldvogel FA, Bisno AL (Hrsg) Infections associated with indwelling medical devices, 3. Aufl. American Society of Microbiology, Washington DC, S 287-306

210. Bachleda P, Kalinova L, Utikal P, Kolar M, Hricova K, Stosova T (2012) Infected prosthetic dialysis arteriovenous grafts: a single dialysis center study. Surg Infect (Larchmt) 13(6):366-370

211. Wang JS, Muzevich K, Edmond MB, Bearman G, Stevens MP (2014) Central nervous system infections due to vancomycin-resistant enterococci: case series and review of the literature. Int J Infect Dis 25:26-31

212. Deutsche Gesellschaft für Anästhesiologie und Intensivmedizin (DGAI), Deutsche Gesellschaft für Infektiologie (DGI), Deutsche Gesellschaft für Hygiene und Mikrobiologie (DGHM), Deutsche Gesellschaft für Pneumologie und Beatmungsmedizin (DGP), Paul-Ehrlich Gesellschaft für Chemotherapie (PEG) (2012) S3-Leitlinie 
020/013 - Epidemiologie, Diagnostik und Therapie erwachsener Patienten mit nosokomialer Pneumonie (gültig bis 30.09.2017). http://www. awmf.org/leitlinien/detail/ll/020-013.html. Zugegriffen: 22. März 2018

213. Johnston PB, Litzow MR, Elliott MA et al (2000) Colonization with vancomycin-resistant enterococcus correlates with poor outcome in patients undergoing allogeneic blood and marrow transplants. Blood 96(11):786a-786a

214. Patel R (2003) Clinical impact of vancomycinresistant enterococci. J Antimicrob Chemother 51(Suppl 3):iii13-iii21

215. Goetz AM, Rihs JD, Wagener MM, Muder RR (1998) Infection and colonization with vancomycin-resistant Enterococcus faecium in an acute care Veterans Affairs Medical Center: a 2-year survey. Am J Infect Control 26(6):558-562

216. Calfee DP, Giannetta ET, Durbin LJ, Germanson TP, Farr BM (2003) Control of endemic vancomycin-resistant Enterococcus among inpatients at a university hospital. Clin Infect Dis 37(3):326-332

217. Fournier S, Brossier F, Fortineau N et al (2012) Long-term control of vancomycin-resistant Enterococcus faecium at the scale of a large multihospital institution: a seven-year experience. Euro Surveill 17(30):11-17

218. Datta R, Huang SS (2010) Risk of postdischarge infection with vancomycin-resistant enterococcus after initial infection or colonization. Infect Control Hosp Epidemiol 31(12):1290-1293

219. Caballero-Granado FJ, Cisneros J, Luque R et al (1998) Comparative study of Bacteremias caused by Enterococcus spp. with and without highlevel resistance to Gentamicin. J Clin Microbiol 36(2):520-525

220. Jiang HL, Zhou Z, Wang LS, Fang Y, Li YH, Cs C (2017) The risk factors, costs, and survival analysis of invasive VRE infections at a medical center in eastern Taiwan. Int J Infect Dis 54:18-24

221. Hefazi M, Damlaj M, Alkhateeb HB et al (2016) Vancomycin-resistant Enterococcus colonization and bloodstream infection: prevalence, risk factors, and the impact on early outcomes after allogeneic hematopoietic cell transplantation in patients with acute myeloid leukemia. Transpl Infect Dis 18(6):913-920

222. Cheah A, Spelman T, Liew D et al (2013) Enterococcal bacteraemia: factors influencing mortality, length of stay and costs of hospitalization. Clin Microbiol Infect 19(4):E181-E189

223. Furtado GH, Mendes RE, Pignatari AC, Wey SB, Medeiros EA (2006) Risk factors for vancomycinresistant Enterococcus faecalis bacteremia in hospitalized patients: an analysis of two casecontrol studies. Am J Infect Control 34(7):447451

224. Flokas ME, Karageorgos SA, Detsis M, Alevizakos M, Mylonakis E (2017) Vancomycin-resistant enterococci colonisation, risk factors and risk for infection among hospitalised paediatric patients: a systematic review and meta-analysis. Int J Antimicrob Agents 49(5):565-572

225. Sutcu M, Akturk H, Acar M et al (2016) Impact of vancomycin-resistant enterococci colonization in critically ill pediatric patients. Am J Infect Control 44(5):515-519

226. Lautenbach E, Bilker WB, Brennan PJ (1999) Enterococcal bacteremia: risk factors for vancomycin resistance and predictors of mortality. Infect Control Hosp Epidemiol 20(05):318-323

227. Vergis EN, Hayden MK, Chow JW et al (2001) Determinants of vancomycin resistance and mortality rates in enterococcal bacteremia. A prospective multicenter study. Ann Intern Med 135(7):484-492

228. Husni R, Hachem R, Hanna H, Raad I (2002) Risk factors for Vancomycin-Resistant Enterococcus (VRE) infection in colonized patients with cancer. Infect Control Hosp Epidemiol 23(2):102-103

229. Morris JG, Shay DK, Hebden JN et al (1995) Enterococci resistant to multiple antimicrobial agents, including vancomycin: establishment of endemicity in a university medical center. Ann Intern Med 123(4):250-259

230. Akturk H, Sutcu M, Somer A et al (2016) Results of four-year rectal Vancomycin-resistant Enterococci surveillance in a pediatric hematologyoncology ward: from colonization to infection. Turk J Haematol 33(3):244-247

231. Institut für Hygiene und Umweltmedizin, Charité Berlin (2017) Outbreak Database. Worldwide Database for Nosocomial Outbreaks. https:// www.outbreak-database.com. Zugegriffen: 22. März 2018

232. Kritsotakis El, Christidou A, Roumbelaki M, Tselentis Y, Gikas A (2008) The dynamic relationship between antibiotic use and the incidence of vancomycin-resistant Enterococcus: time-series modelling of 7-year surveillance data in a tertiary-care hospital. Clin Microbiol Infect 14(8):747-754

233. Clutter DS, Dubrovskaya Y, Merl MY, Teperman L, Press R, Safdar A (2013) Fidaxomicin versus conventional antimicrobial therapy in 59 recipients of solid organ and hematopoietic stem cell transplantation with Clostridium difficile-associated diarrhea. Antimicrob Agents Chemother 57(9):4501-4505

234. Akturk H, Sutcu M, Somer A et al (2016) Vancomycin-resistant enterococci colonization in a neonatal intensive care unit: who will be infected? J Matern Fetal Neonatal Med 29(21):3478-3482

235. Safdar N, Maki DG (2002) The commonality of risk factors for nosocomial colonization and infection with antimicrobial-resistant Staphylococcus aureus, enterococcus, gram-negative bacilli, Clostridium difficile, and Candida. Ann Intern Med 136(11):834-844

236. Papadimitriou-Olivgeris $M$, Drougka E, Fligou $F$ et al (2014) Risk factors for enterococcal infection and colonization by vancomycin-resistant enterococci in critically ill patients. Infection 42(6):1013-1022

237. Shaked H, Carmeli Y, Schwartz D, Siegman-Igra Y (2006) Enterococcal bacteraemia: epidemiological, microbiological, clinical and prognostic characteristics, and the impact of high level gentamicin resistance. Scand J Infect Dis 38(1112):995-1000

238. Watanakunakorn C, Patel R (1993) Comparison of patients with enterococcal bacteremia due to strains with and without high-level resistance to gentamicin. Clin Infect Dis 17(1):74-78

239. Jang HC, Lee S, Song KH et al (2010) Clinical features, risk factors and outcomes of bacteremia due to enterococci with high-level gentamicin resistance: comparison with bacteremia due to enterococci without high-level gentamicin resistance. J Korean Med Sci 25(1):3-8

240. McBride S, Upton A, Roberts S (2010) Clinical characteristics and outcomes of patients with vancomycin-susceptible Enterococcus faecalis and Enterococcus faecium bacteraemia-a five- year retrospective review. Eur J Clin Microbiol Infect Dis 29(1):107-114

241. Tripathi A, Shukla S, Singh A, Prasad K (2016) Prevalence, outcome and risk factor associated with vancomycin-resistant Enterococcus faecalis and Enterococcus faecium at a Tertiary Care Hospital in Northern India. Indian J Med Microbiol 34(1):38

242. Jung E, Byun S, Lee H, Moon SY, Lee H (2014) Vancomycin-resistant Enterococcus colonization in the intensive care unit: clinical outcomes and attributable costs of hospitalization. Am J Infect Control 42(10):1062-1066

243. Puchter L, Chaberny IF, Schwab F, Vonberg RP, Bange FC, Ebadi E (2018) Economic burden of nosocomial infections caused by vancomycinresistant enterococci. Antimicrob Resist Infect Control 7:1

244. Britt NS, Potter EM, Patel N, Steed ME (2015) Comparison of the effectiveness and safety of linezolid and daptomycin in vancomycin-resistant enterococcal bloodstream infection: a national cohort study of Veterans Affairs patients. Clin Infect Dis 61(6):871-878

245. Chuang Y, Wang J, Lin H, Chang S (2014) Daptomycin versus linezolid for treatment of vancomycin-resistant enterococcal bacteremia: systematic review and meta-analysis. BMC Infect Dis 14(1):687

246. Balli EP, Venetis CA, Miyakis S (2014) Systematic review and meta-analysis of linezolid versus daptomycin for treatment of vancomycinresistant enterococcal bacteremia. Antimicrob Agents Chemother 58(2):734-739

247. Ornstein MC, Mukherjee S, Keng M et al (2015) Impact of vancomycin-resistant enterococcal bacteremia on outcome during acute myeloid leukemia induction therapy. Leuk Lymphoma 56(9):2536-2542

248. Prematunge $C$, MacDougall C, Johnstone J et al (2016) VRE and VSE bacteremia outcomes in the era of effective VRE therapy: a systematic review and meta-analysis. Infect Control Hosp Epidemiol 37(01):26-35

249. Khair $H$, VanTassell $P$, Henderson J, Warren $D$ Marschall J, Program CPE (2013) Vancomycin resistance has no influence on outcomes of enterococcal bacteriuria. J Hosp Infect 85(3):183188

250. Pintado V, Cabellos C, Moreno S, Meseguer MA, Ayats J, Viladrich PF (2003) Enterococcal meningitis: a clinical study of 39 cases and review of the literature. Medicine (Baltimore) 82(5):346-364

251. Forrest GN, Arnold RS, Gammie JS, Gilliam BL (2011) Single center experience of a vancomycin resistant enterococcal endocarditis cohort. J Infect 63(6):420-428

252. Bucheli E, Kralidis G, Boggian K et al (2014) Impact of enterococcal colonization and infection in solid organ transplantation recipients from the Swiss transplant cohort study. Transpl Infect Dis 16(1):26-36

253. Kim YJ, Kim SI, Choi JY, Yoon SK, You YK, Kim DG (2015) Clinical significance of methicillin-resistant Staphylococcus aureus and vancomycinresistant enterococci colonization in liver transplant recipients. Korean J Intern Med 30(5):694-704

254. Satlin MJ, Soave R, Racanelli AC et al (2014) The emergence of vancomycin-resistant enterococcal bacteremia in hematopoietic 
stem cell transplant recipients. Leuk Lymphoma 55(12):2858-2865

255. Vydra J, Shanley RM, George I et al (2012) Enterococcal bacteremia is associated with increased risk of mortality in recipients of allogeneic hematopoietic stem cell transplantation. Clin Infect Dis 55(6):764-770

256. Tavadze M, Rybicki L, Mossad S et al (2014) Risk factors for vancomycin-resistant enterococcus bacteremia and its influence on survival after allogeneic hematopoietic cell transplantation. Bone Marrow Transplant 49(10):1310-1316

257. Adams DJ, Eberly MD, Goudie A, Nylund CM (2016) Rising Vancomycin-resistant Enterococcus infections in hospitalized children in the United States. Hosp Pediatr 6(7):404-411

258. Chou YY, Lin TY, Lin JC, Wang NC, Peng MY, Chang FY (2008) Vancomycin-resistant enterococcal bacteremia: comparison of clinical features and outcome between Enterococcus faecium and Enterococcus faecalis. J Microbiol Immunol Infect 41(2):124-129

259. Hayakawa K, Marchaim D, Martin ET et al (2012) Comparison of the clinical characteristics and outcomes associated with vancomycin-resistant Enterococcus faecalis and vancomycin-resistant E. faecium bacteremia. Antimicrob Agents Chemother 56(5):2452-2458

260. Hayakawa K, Martin ET, Gudur UM et al (2014) The impact of different antimicrobial therapies on clinical and fiscal outcomes of patients with bacteremia due to vancomycin-resistant enterococci. Antimicrob Agents Chemother 58(7):3968-3975

261. Britt NS, Potter EM (2016) Clinical epidemiology of vancomycin-resistant Enterococcus gallinarum and Enterococcus casseliflavus bloodstream infections. J Glob Antimicrob Resist 5:57-61

262. Worth LJ, Thursky KA, Seymour JF, Slavin MA (2007) Vancomycin-resistant Enterococcus faecium infection in patients with hematologic malignancy: patients with acute myeloid leukemia are at high-risk. Eur J Haematol 79(3):226-233

263. Pogue JM, Paterson DL, Pasculle AW, Potoski BA (2007) Determination of risk factors associated with isolation of Linezolid-resistant strains of Vancomycin-resistant Enterococcus. Infect Control Hosp Epidemiol 28(12):1382-1388

264. Malczynski M, Qi C (2010) Differences in clinical outcomes in patients with vancomycin-resistant enterococci according to linezolid susceptibility. Pharmacotherapy 30(12):1221-1228

265. Santayana EM, Grim SA, Janda WM, Layden JE, Lee TA, Clark NM (2012) Risk factors and outcomes associated with vancomycin-resistant Enterococcus infections with reduced susceptibilities to linezolid. Diagn Microbiol Infect Dis 74(1):39-42

266. Hayakawa K, Marchaim D, Pogue JM et al (2012) Predictors and outcomes of linezolid-resistant vancomycin-resistant Enterococcus: a case-casecontrol study. Am J Infect Control 40(10):e261263

267. Jia X, Ma W, Xu X, Yang S, Zhang L (2015) Retrospective analysis of hospital-acquired linezolid-nonsusceptible enterococci infection in Chongqing, China, 2011-2014. Am J Infect Control 43(12):e101-e106

268. Pai MP, Rodvold KA, Schreckenberger PC, Gonzales RD, Petrolatti JM, Quinn JP (2002) Risk factors associated with the development of infection with linezolid-and vancomycin-resistant Enterococcus faecium. Clin Infect Dis 35(10):1269-1272
269. Kainer MA (2007) Response to emerging infection leading to outbreak of Linezolid-resistant Enterococci. Emerging Infect Dis 13(7):10241030

270. Cordina C, Hill R, Deshpande A, Hood J, Inkster $T$ (2012) Tigecycline-resistant Enterococcus faecalis associated with omeprazole use in a surgical patient. J Antimicrob Chemother 67(7):1806-1807

271. Kelesidis T, Humphries R, Uslan DZ, Pegues DA (2011) Daptomycin nonsusceptible enterococci: an emerging challenge for clinicians. Clin Infect Dis 52(2):228-234

272. Hidron Al, Schuetz AN, Nolte FS, Gould CV, Osborn MK (2008) Daptomycin resistance in Enterococcus faecalis prosthetic valve endocarditis. J Antimicrob Chemother 61(6):1394-1396

273. Kanafani ZA, Federspiel JJ, Fowler VG Jr (2007) Infective endocarditis caused by daptomycinresistant Enterococcus faecalis: a case report. Scand J Infect Dis 39(1):75-77

274. Green MR, Anasetti C, Sandin RL, Rolfe NE, Greene JN (2006) Development of daptomycin resistance in a bone marrow transplant patient with vancomycin-resistant Enterococcus durans. J Oncol Pharm Pract 12(3):179-181

275. Sabol K, Patterson JE, Lewis JS, Owens A, Cadena J, Jorgensen JH (2005) Emergence of daptomycin resistance in Enterococcus faecium during daptomycin therapy. Antimicrob Agents Chemother 49(4):1664-1665

276. Munoz-Price LS, Lolans K, Quinn JP (2005) Emergence of resistance to daptomycin during treatment of vancomycin-resistant Enterococcus faecalis infection. Clin Infect Dis 41(4):565-566

277. Long JK, Choueiri TK, Hall GS, Avery RK, Sekeres MA (2005) Daptomycin-resistant Enterococcus faecium in a patient with acute myeloid leukemia. Mayo Clin Proc 80(9):1215-1216

278. Arias CA, Torres HA, Singh KV et al (2007) Failure of daptomycin monotherapy for endocarditis caused by an Enterococcus faecium strain with vancomycin-resistant and vancomycinsusceptible subpopulations and evidence of in vivo loss of the vanA gene cluster. Clin Infect Dis 45(10):1343-1346

279. Kamboj M, Cohen N, Gilhuley K, Babady NE Seo SK, Sepkowitz KA (2011) Emergence of daptomycin-resistant VRE: experience of a single institution. Infect Control Hosp Epidemiol 32(04):391-394

280. Kelesidis T, Humphries R, Uslan D, Pegues D (2012) De Novo Daptomycin-Nonsusceptible Enterococcal Infections. Emerging Infect Dis 18(4):674-676

281. Wudhikarn K, Gingrich RD, de Magalhaes Silverman M (2013) Daptomycin nonsusceptible enterococci in hematologic malignancy and hematopoietic stem cell transplant patients: an emerging threat. Ann Hematol 92(1):129-131

282. Lewis J, Enfield K, Cox H, Mathers A, Sifri C (2016) A single-center experience with infections due to daptomycin-nonsusceptible Enterococcus faecium in liver transplant recipients. Transpl Infect Dis 18(3):341-353

283. Herc ES, Kauffman CA, Marini BL, Perissinotti AJ, Miceli MH (2017) Daptomycin nonsusceptible vancomycin resistant Enterococcus bloodstream infections in patients with hematological malignancies: risk factors and outcomes. Leuk Lymphoma 58(12):2852-2858

284. Chenoweth CE, Bradley SF, Terpenning MS et al (1994) Colonization and transmission of high- level gentamicin-resistant enterococci in a longterm care facility. Infect Control Hosp Epidemiol 15(11):703-709

285. Derde LPG, Cooper BS, Goossens H et al (2014) Interventions to reduce colonisation and transmission of antimicrobial-resistant bacteria in intensive care units: an interrupted time series study and cluster randomised trial. Lancet Infect Dis 14(1):31-39

286. Fisher D, Pang L, Salmon S et al (2016) A successful vancomycin-resistant enterococci reduction bundle at a Singapore hospital. Infect Control Hosp Epidemiol 37(01):107-109

287. Popiel KY, Miller MA (2014) Evaluation of Vancomycin-Resistant Enterococci (VRE)-Associated Morbidity Following Relaxation of VRE Screening and Isolation Precautions in a Tertiary Care Hospital. Infect Control Hosp Epidemiol 35(07):818-825

288. Montecalvo MA, Jarvis WR, Uman J et al (1999) Infection-control measures reduce transmission of vancomycin-resistant enterococci in an endemic setting. Ann Intern Med 131(4):269-272

289. Price CS, Paule S, Noskin GA, Peterson LR (2003) Active surveillance reduces the incidence of vancomycin-resistant enterococcal bacteremia. Clin Infect Dis 37(7):921-928

290. Ostrowsky BE, Trick WE, Sohn AH et al (2001) Control of vancomycin-resistant enterococcus in health care facilities in a region. $\mathrm{N}$ Engl J Med 344(19):1427-1433

291. Garbutt JM, Littenberg B, Evanoff BA, Sahm D, Mundy LM (1999) Enteric carriage of vancomycin-resistant Enterococcus faecium in patients tested for Clostridium difficile. Infect Control Hosp Epidemiol 20(10):664-670

292. Özsoy S, Ilki A (2017) Detection of vancomycinresistant enterococci (VRE) in stool specimens submitted for Clostridium difficile toxin testing. Braz J Microbiol 48(3):489-492

293. Leber AL, Hindler JF, Kato EO, Bruckner DA Pegues DA (2001) Laboratory-based surveillance for vancomycin-resistant enterococci: utility of screening stool specimens submitted for Clostridium difficile toxin assay. Infect Control Hosp Epidemiol 22(3):160-164

294. Bodily M, McMullen KM, Russo AJ, Kittur ND, Hoppe-Bauer J, Warren DK (2013) Discontinuation of reflex testing of stool samples for vancomycin-resistant enterococci resulted in increased prevalence. Infect Control Hosp Epidemiol 34(8):838-840

295. Munigala S, McMullen KM, Russo AJ et al (2017) Reinstatement of reflex testing of stool samples for Vancomycin-Resistant Enterococci (VRE) resulted in decreased incidence of hospitalassociated VRE. Infect Control Hosp Epidemiol 38(5):619-621

296. Cetinkaya Y, Falk P, Mayhall CG (2000) Vancomycin-resistant enterococci. Clin Microbiol Rev 13(4):686-707

297. D’Agata EMD, Gautam S, Green WK, Tang Y-W (2002) High rate of false-negative results of the rectal swab culture method in detection of gastrointestinal colonization with vancomycin-resistant enterococci. Clin Infect Dis 34(2):167-172

298. Warnke P, Warning L, Podbielski A (2014) Some are more equal - A comparative study on swab uptake and release of bacterial suspensions. PLoS ONE 9(7):e102215

299. Pearman JW (2006) 2004 Lowbury Lecture: the Western Australian experience with vanco- 
mycin-resistant enterococci - from disaster to ongoing control. J Hosp Infect 63(1):14-26

300. Drews SJ, Johnson G, Gharabaghi F et al (2006) A 24-hour screening protocol for identification of vancomycin-resistant Enterococcus faecium. J Clin Microbiol 44(4):1578-1580

301. Wade JJ (1995) The emergence of Enterococcus faecium resistant to glycopeptides and other standard agents - a preliminary report. J Hosp Infect 30:483-493

302. Novicki TJ, Schapiro JM, Ulness BK et al (2004) Convenient selective differential broth for isolation of vancomycin-resistant Enterococcus from fecal material. J Clin Microbiol 42(4):1637-1640

303. Stamper PD, Shulder S, Bekalo P et al (2010) Evaluation of BBL CHROMagar VanRE for detection of vancomycin-resistant Enterococci in rectal swab specimens. J Clin Microbiol 48(11):42944297

304. Malhotra-Kumar S, Haccuria K, Michiels M et al (2008) Current trends in rapid diagnostics for methicillin-resistant Staphylococcus aureus and glycopeptide-resistant enterococcus species. J Clin Microbiol 46(5):1577-1587

305. Suwantarat N, Roberts A, Prestridge J et al (2014) Comparison of five chromogenic media for recovery of vancomycin-resistant enterococci from fecal samples. J Clin Microbiol 52(11):4039-4042

306. Brown DF, Walpole E (2003) Evaluation of selective and enrichment media for isolation of glycopeptide-resistant enterococci from faecal specimens. J Antimicrob Chemother 51(2):289-296

307. Werner G, Serr A, Schütt S et al (2011) Comparison of direct cultivation on a selective solid medium, polymerase chain reaction from an enrichment broth, and the BD GeneOhm ${ }^{\mathrm{TM}}$ VanR Assay for identification of vancomycin-resistant enterococci in screening specimens. Diagn Microbiol Infect Dis 70(4):512-521

308. Papadimitriou-Olivgeris M, Filippidou S, Kolonitsiou F et al (2016) Pitfalls in the identification of Enterococcus species and the detection of vanA and vanB genes. Lett Appl Microbiol 63(3):189-195

309. Babady NE, Gilhuley K, Cianciminio-Bordelon D, Tang YW (2012) Performance characteristics of the Cepheid Xpert vanA assay for rapid identification of patients at high risk for carriage of vancomycin-resistant Enterococci. J Clin Microbiol 50(11):3659-3663

310. Franke GC, Klupp EM, Mirwald N et al (2015) Real life evaluation of VRE vanA/vanB real-time PCR for surveillance of vancomycin-resistant enterococci in rectal swab samples. P0767. Poster session presented at the meeting of the ESCMID, Copenhagen.,

311. Lucet JC, Armand-Lefevre L, Laurichesse JJ et al (2007) Rapid control of an outbreak of vancomycin-resistant enterococci in a French university hospital. J Hosp Infect 67(1):42-48

312. Wang JT, Chen YC, Chang SC et al (2004) Control of vancomycin-resistant enterococci in a hospital: a five-year experience in a Taiwanese teaching hospital. J Hosp Infect 58(2):97-103

313. Bearman GM, Marra AR, Sessler CN et al (2007) A controlled trial of universal gloving versus contact precautions for preventing the transmission of multidrug-resistant organisms. Am J Infect Control 35(10):650-655

314. Bearman G, Rosato AE, Duane TM et al (2010) Trial of universal gloving with emollientimpregnated gloves to promote skin health and prevent the transmission of multidrug-resistant organisms in a surgical intensive care unit. Infect Control Hosp Epidemiol 31(5):491-497

315. Harris AD, Pineles L, Belton B et al (2013) Universal glove and gown use and acquisition of antibiotic-resistant bacteria in the ICU: a randomized trial. JAMA 310(15):1571-1580

316. Aboelela SW, Saiman L, Stone P, Lowy FD, Quiros D, Larson E (2006) Effectiveness of barrier precautions and surveillance cultures to control transmission of multidrug-resistant organisms: a systematic review of the literature. Am J Infect Control 34(8):484-494

317. Cohen C, Cohen B, Shang J (2015) Effectiveness of contact precautions against multidrugresistant organism transmission in acute care: a systematic review of the literature. J Hosp Infect 90(4):275-284

318. De Angelis G, Cataldo MA, De Waure C et al (2014) Infection control and prevention measures to reduce the spread of vancomycin-resistant enterococci in hospitalized patients: a systematic review and meta-analysis. J Antimicrob Chemother 69(5):1185-1192

319. Edmond MB, Masroor N, Stevens MP, Ober J, Bearman G (2015) The impact of discontinuing contact precautions for VRE and MRSA on device-associated infections. Infect Control Hosp Epidemiol 36(8):978-980

320. Almyroudis NG, Osawa R, Samonis G et al (2016) Discontinuation of systematic surveillance and contact precautions for Vancomycin-Resistant Enterococcus (VRE) and its impact on the incidence of VRE faecium Bacteremia in patients with hematologic malignancies. Infect Control Hosp Epidemiol 37(4):398-403

321. Bardossy AC, Alsafadi MY, Starr P et al (2017) Evaluation of contact precautions for methicillin-resistant Staphylococcus aureus and vancomycin-resistant Enterococcus. Am J Infect Control 45(12):1369-1371

322. Marra AR, Edmond MB, Schweizer ML, Ryan GW, Diekema DJ (2018) Discontinuing contact precautions for multidrug-resistant organisms: a systematic literature review and meta-analysis. Am J Infect Control 46(3):333-340

323. Armeanu E, Bonten MJ (2005) Control of vancomycin-resistant enterococci: one size fits all? Clin Infect Dis 41(2):210-216

324. Venkatesh AK, Lankford MG, Rooney DM, Blachford T, Watts CM, Noskin GA (2008) Use of electronic alerts to enhance hand hygiene compliance and decrease transmission of vancomycin-resistant Enterococcus in a hematology unit. Am J Infect Control 36(3):199-205

325. Jayaraman SP, Klompas M, Bascom M et al (2014) Hand-hygiene compliance does not predict rates of resistant infections in critically ill surgical patients. Surg Infect (Larchmt) 15(5):533-539

326. Tenorio AR, Badri SM, Sahgal NB et al (2001) Effectiveness of gloves in the prevention of hand carriage of vancomycin-resistant enterococcus species by health care workers after patient care. Clin Infect Dis 32(5):826-829

327. Puzniak LA, Leet T, Mayfield J, Kollef M, Mundy LM (2002) To gown or not to gown: the effect on acquisition of vancomycin-resistant enterococci. Clin Infect Dis 35(1):18-25

328. Srinivasan A, Song X, Ross T, Merz W, Brower R, Perl TM (2002) A prospective study to determine whether cover gowns in addition to gloves decrease nosocomial transmission of vancomycin- resistant enterococci in an intensive care unit. Infect Control Hosp Epidemiol 23(8):424-428

329. Perugini MR, Nomi SM, Lopes GK et al (2011) Impact of the reduction of environmental and equipment contamination on vancomycin-resistant enterococcus rates. Infection 39(6):587-593

330. Cheng VC, Tai JW, Chau P et al (2016) Successful control of emerging vancomycin-resistant enterococci by territory-wide implementation of directly observed hand hygiene in patients in Hong Kong. Am J Infect Control 44(10):11681171

331. Bradley SJ, Wilson AL, Allen MC, Sher HA, Goldstone AH, Scott GM (1999) The control of hyperendemic glycopeptide-resistant Enterococcus spp. on a haematology unit by changing antibiotic usage. J Antimicrob Chemother 43(2):261-266

332. Cohen B, Cohen CC, Loyland B, Larson EL (2017) Transmission of health care-associated infections from roommates and prior room occupants: a systematic review. Clin Epidemiol 9:297-310

333. Knelson LP, Williams DA, Gergen MF et al (2014) A comparison of environmental contamination by patients infected or colonized with methicillin-resistant Staphylococcus aureus or vancomycin-resistant enterococci: a multicenter study. Infect Control Hosp Epidemiol 35(7):872-875

334. Ford CD, Lopansri BK, Gazdik MA et al (2016) Room contamination, patient colonization pressure, and the risk of vancomycin-resistant Enterococcus colonization on a unit dedicated to the treatment of hematologic malignancies and hematopoietic stem cell transplantation. Am J Infect Control 44(10):1110-1115

335. Falagas M, Thomaidis P, Kotsantis I, Sgouros K, Samonis G, Karageorgopoulos D (2011) Airborne hydrogen peroxide for disinfection of the hospital environment and infection control: a systematic review. J Hosp Infect 78(3):171-177

336. Passaretti CL, Otter JA, Reich NG et al (2013) An evaluation of environmental decontamination with hydrogen peroxide vapor for reducing the risk of patient acquisition of multidrug-resistant organisms. Clin Infect Dis 56(1):27-35

337. Horn K, Otter JA (2015) Hydrogen peroxide vapor room disinfection and hand hygiene improvements reduce Clostridium difficile infection, methicillin-resistant Staphylococcus aureus, vancomycin-resistant enterococci, and extended-spectrum beta-lactamase. Am J Infect Control 43(12):1354-1356

338. Reichenbacher D, Thanheiser M, Krüger D (2010) Aktueller Stand zur Raumdekontamination mit gasförmigem Wasserstoffperoxid Status quo of room decontamination by vaporized hydrogen peroxide. Hyg Med 35(6):204-208

339. Robert Koch-Institut (RKI) (2017) 3.3. Raumdesinfektion. In: Robert Koch-Institut (RKI) Liste der vom Robert Koch-Institut geprüften und anerkannten Desinfektionsmittel und -verfahren (17. Ausgabe). Bundesgesundheitsblatt 60(11):1274-1297

340. Byrns G, Fuller TP (2011) The risks and benefits of chemical fumigation in the health care environment. J Occup Environ Hyg 8(2):104-112

341. Popp W (2014) Probleme bei der Etablierung eines Wasserstoffperoxid-Verneblers. Hyg Med 39(3):77-80

342. Grabsch EA, Mahony AA, Cameron DR et al (2012) Significant reduction in vancomycin- 
resistant enterococcus colonization and bacteraemia after introduction of a bleach-based cleaning-disinfection programme. J Hosp Infect 82(4):234-242

343. Bryce E, Grant J, Scharf S et al (2015) Horizontal infection prevention measures and a riskmanaged approach to vancomycin-resistant enterococci: an evaluation. Am J Infect Control 43(11):1238-1243

344. Brooks S, Khan A, Stoica D et al (1998) Reduction in vancomycin-resistant Enterococcus and Clostridium difficile infections following change to tympanic thermometers. Infect Control Hosp Epidemiol 19(5):333-336

345. Climo MW, Sepkowitz KA, Zuccotti G et al (2009) The effect of daily bathing with chlorhexidine on the acquisition of methicillin-resistant Staphylococcus aureus, vancomycin-resistant Enterococcus, and healthcare-associated bloodstream infections: results of a quasi-experimental multicenter trial. Crit Care Med 37(6):1858-1865

346. Kassakian SZ, Mermel LA, Jefferson JA, Parenteau SL, Machan JT (2011) Impact of Chlorhexidine bathing on hospital-acquired infections among general medical patients. Infect Control Hosp Epidemiol 32(3):238-243

347. Gastmeier P, Kämpf K-P, Behnke M, Geffers C, Schwab F (2016) An observational study of the universal use of octenidine to decrease nosocomial bloodstream infections and MDR organisms. J Antimicrob Chemother 71(9):25692576

348. Chen WS, Li SQ, Li LH, Wu X, Zhang WH (2013) Effects of daily bathing with chlorhexidine and acquired infection of methicillin-resistant Staphylococcus aureus and vancomycin-resistant Enterococcus: a meta-analysis. J Thorac Dis 5(4):518-524

349. Dicks KV, Lofgren E, Lewis SS, Moehring RW, Sexton DJ, Anderson DJ (2016) A multicenter pragmatic interrupted time series analysis of Chlorhexidine Gluconate bathing in community hospital intensive care units. Infect Control Hosp Epidemiol 37(7):791-797

350. Martin EM, Russell D, Rubin Z et al (2016) Elimination of routine contact precautions for endemic Methicillin-resistant staphylococcus aureus and Vancomycin-Resistant Enterococcus: a retrospective quasi-experimental study. Infect Control Hosp Epidemiol 37(11):1323-1330

351. Guzman Prieto AM, Wijngaarden J, Braat JC et al (2017) The two-component system ChtRS contributes to Chlorhexidine tolerance in Enterococcus faecium. Antimicrob Agents Chemother 61(5):e02122-16

352. O'Donovan CA, Fan-Havard P, Tecson-Tumang FT, Smith SM, Eng RH (1994) Enteric eradication of vancomycin-resistant Enterococcus faecium with oral bacitracin. Diagn Microbiol Infect Dis 18(2):105-109

353. Weinstein MR, Dedier H, Brunton J, Campbell I, Conly JM (1999) Lack of efficacy of oral bacitracin plus doxycycline for the eradication of stool colonization with vancomycin-resistant Enterococcus faecium. Clin Infect Dis 29(2):361-366

354. Brossier F, Lefrancois S, Paute J et al (2010) Decolonisation for early control of an outbreak of vancomycin-resistant Enterococcus faecium in a geriatric rehabilitation care facility. J Hosp Infect 76(4):368-369

355. Chia JK, Nakata MM, Park SS, Lewis RP, McKee B (1995) Use of bacitracin therapy for infection due to vancomycin-resistant Enterococcus faecium. Clin Infect Dis 21(6):1520

356. Mondy KE, Shannon W, Mundy LM (2001) Evaluation of zinc bacitracin capsules versus placebo for enteric eradication of vancomycinresistant Enterococcus faecium. Clin Infect Dis 33(4):473-476

357. Hachem R, Raad I (2002) Failure of oral antimicrobial agents in eradicating gastrointestinal colonization with vancomycin-resistant enterococci. Infect Control Hosp Epidemiol 23(1):43-44

358. Wong MT, Kauffman CA, Standiford HC et al (2001) Effective suppression of vancomycinresistant Enterococcus species in asymptomatic gastrointestinal carriers by a novel glycolipodepsipeptide, ramoplanin. Clin Infect Dis 33(9):1476-1482

359. Montecalvo MA (2003) Ramoplanin: a novel antimicrobial agent with the potential to prevent vancomycin-resistant enterococcal infection in high-risk patients. J Antimicrob Chemother 51(Suppl 3):iii31-iii35

360. Montecalvo MA, Horowitz $\mathrm{H}$, Wormser GP, Seiter K, Carbonaro CA (1995) Effect of novobiocincontaining antimicrobial regimens on infection and colonization with vancomycin-resistant Enterococcus faecium. Antimicrob Agents Chemother 39(3):794

361. Heineman J, Bubenik S, McClave S, Martindale $R$ (2012) Fighting fire with fire: is it time to use probiotics to manage pathogenic bacterial diseases? Curr Gastroenterol Rep 14(4):343-348

362. Manley KJ, Fraenkel MB, Mayall BC, Power DA (2007) Probiotic treatment of vancomycinresistant enterococci: a randomised controlled trial. Med J Aust 186(9):454-457

363. Szachta P, Ignys I, Cichy W (2011) An evaluation of the ability of the probiotic strain Lactobacillus rhamnosus GG to eliminate the gastrointestinal carrier state of vancomycin-resistant enterococci in colonized children. J Clin Gastroenterol 45(10):872-877

364. de Regt MJ, Willems RJ, Hene RJ et al (2010) Effects of probiotics on acquisition and spread of multiresistant enterococci. Antimicrob Agents Chemother 54(7):2801-2805

365. Warrack S, Ziegler M, Duster M, Panjikar P, Safdar $\mathrm{N}$ (2016) A pilot randomized trial to determine the tolerability of a Probiotic in patients colonized with Vancomycin-resistant Enterococcus. J Prob Health 4(2):142

366. Doron S, Snydman DR (2015) Risk and safety of probiotics. Clin Infect Dis 60(Suppl 2):S129-S134

367. Haghighat L, Crum-Cianflone NF (2016) The potential risks of probiotics among HIV-infected persons: Bacteraemia due to lactobacillus acidophilus and review of the literature. Int J Std Aids 27(13):1223-1230

368. Zbinden A, Zbinden R, Berger C, Arlettaz R (2015) Case series of Bifidobacterium longum bacteremia in three preterm infants on probiotic therapy. Neonatology 107(1):56-59

369. Lund B, Edlund C (2001) Probiotic Enterococcus faecium strain is a possible recipient of the vanA gene cluster. Clin Infect Dis 32(9):1384-1385

370. Ubeda C, Bucci V, Caballero S et al (2013) Intestinal microbiota containing Barnesiella species cures vancomycin-resistant Enterococcus faecium colonization. Infect Immun 81(3):965-973

371. Davido B, Batista R, Fessi H, Salomon J, Dinh A (2017) Impact of faecal microbiota transplantation to eradicate vancomycin-resistant en- terococci (VRE) colonization in humans. J Infect 75(4):376-377

372. Sohn KM, Cheon S, Kim YS (2016) Can Fecal Microbiota Transplantation (FMT) Eradicate Fecal Colonization With Vancomycin-Resistant Enterococci (VRE)? Infect Control Hosp Epidemiol 37(12):1519-1521

373. Tacconelli E, De Angelis G, Cataldo MA et al (2009) Antibiotic usage and risk of colonization and infection with antibiotic-resistant bacteria: a hospital population-based study. Antimicrob Agents Chemother 53(10):4264-4269

374. McKinnell JA, Kunz DF, Chamot E et al (2012) Association between vancomycin-resistant Enterococci bacteremia and ceftriaxone usage. Infect Control Hosp Epidemiol 33(7):718-724

375. Gouliouris T, Warne B, Cartwright EJP et al (2018) Duration of exposure to multiple antibiotics is associated with increased risk of VRE bacteraemia: a nested case-control study. J Antimicrob Chemother 73(6):1692-1699

376. Mauldin PD, Salgado CD, Durkalski VL, Bosso JA (2008) Nosocomial infections due to methicillin-resistant Staphylococcus aureus and vancomycin-resistant enterococcus: relationships with antibiotic use and cost drivers. Ann Pharmacother 42(3):317-326

377. McKinnell JA, Kunz DF, Moser SA et al (2016) Patient-level analysis of incident vancomycinresistant enterococci colonization and antibiotic days of therapy. Epidemiol Infect 144(8):17481755

378. Hospital Infection Control Practices Advisory Committee (HICPAC) (1995) Recommendations for preventing the spread of vancomycin resistance. MMWR Recomm Rep 44(RR-12):1-13

379. Belliveau PP, Rothman AL, Maday CE (1996) Limiting vancomycin use to combat vancomycinresistant Enterococcus faecium. Am J Health Syst Pharm 53(13):1570-1575

380. Shaikh ZH, Osting CA, Hanna HA, Arbuckle RB, Tarr JJ, Raad II (2002) Effectiveness of a multifaceted infection control policy in reducing vancomycin usage and vancomycin-resistant enterococci at a tertiary care cancer centre. J Hosp Infect 51(1):52-58

381. Anglim AM, Klym B, Byers KE, Scheld WM, Farr BM (1997) Effect of a vancomycin restriction policy on ordering practices during an outbreak of vancomycin-resistant Enterococcus faecium. Arch Intern Med 157(10):1132-1136

382. Meyer E, Schwab F, Schroeren-Boersch B, Gastmeier P (2010) Dramatic increase of third-generation cephalosporin-resistant E. coli in German intensive care units: secular trends in antibiotic drug use and bacterial resistance, 2001 to 2008. Crit Care 14(3):R113

383. Smith RL, Evans HL, Chong TW et al (2008) Reduction in rates of methicillin-resistant Staphylococcus aureus infection after introduction of quarterly linezolid-vancomycin cycling in a surgical intensive care unit. Surg Infect (Larchmt) 9(4):423-431

384. May AK, Melton SM, McGwin G, Cross JM, Moser SA, Rue LW (2000) Reduction of vancomycinresistant enterococcal infections by limitation of broad-spectrum cephalosporin use in a trauma and burn intensive care unit. Shock 14(3):259264

385. Lautenbach E, LaRosa LA, Marr AM, Nachamkin I, Bilker WB, Fishman NO (2003) Changes in the prevalence of vancomycin-resistant enterococci in response to antimicrobial formulary 
interventions: impact of progressive restrictions on use of vancomycin and third-generation cephalosporins. Clin Infect Dis 36(4):440-446

386. Arda B, Sipahi OR, Yamazhan T et al (2007) Short-term effect of antibiotic control policy on the usage patterns and cost of antimicrobials, mortality, nosocomial infection rates and antibacterial resistance. J Infect 55(1):41-48

387. Peto Z, Benko R, Matuz M, Csullog E, Molnar A, Hajdu E (2008) Results of a local antibiotic management program on antibiotic use in a tertiary intensive care unit in Hungary. Infection 36(6):560-564

388. Jaggi N, Sissodia P, Sharma L (2012) Control of multidrug resistant bacteria in a tertiary care hospital in India. Antimicrob Resist Infect Control 1(1):23

389. de Bruin MA, Riley LW (2007) Does vancomycin prescribing intervention affect vancomycin-resistant enterococcus infection and colonization in hospitals? A systematic review. BMC Infect Dis 7:24

390. Tacconelli E (2009) Antimicrobial use: risk driver of multidrug resistant microorganisms in healthcare settings. Curr Opin Infect Dis 22(4):352-358

391. Baur D, Gladstone BP, Burkert F et al (2017) Effect of antibiotic stewardship on the incidence of infection and colonisation with antibiotic-resistant bacteria and Clostridium difficile infection: a systematic review and meta-analysis. Lancet Infect Dis 17(9):990-1001

392. Climo MW, Yokoe DS, Warren DK et al (2013) Effect of daily Chlorhexidine bathing on hospitalacquired infection. N Engl J Med 368(6):533-542

393. Musuuza JS, Sethi AK, Roberts TJ, Safdar N (2017) Implementation of daily chlorhexidine bathing to reduce colonization by multidrug-resistant organisms in a critical care unit. Am J Infect Control 45(9):1014-1017

394. Frakking FNJ, Bril WS, Sinnige JC et al (2018) Recommendations for the successful control of a large outbreak of vancomycin-resistant Enterococcus faecium in a non-endemic hospital setting. J Hosp Infect. https://doi.org/10.1016/j. jhin.2018.02.016

395. Slaughter S, Hayden MK, Nathan C et al (1996) A comparison of the effect of universal use of gloves and gowns with that of glove use alone on acquisition of vancomycin-resistant enterococci in a medical intensive care unit. Ann Intern Med 125(6):448-456

396. Gandra S, Barysauskas C, Mack DA, Barton B, Finberg R, Ellison RT III (2014) Impact of contact precautions on falls, pressure ulcers and transmission of MRSA and VRE in hospitalized patients. J Hosp Infect 88(3):170-176

397. Rupp ME, Fitzgerald T, Hayes Ket al (2017) Effect of cessation of contact isolation for endemic methicillin-resistant staphylococcus aureus and vancomycin-resistant enterococci. Infect Control Hosp Epidemiol 38(8):1005-1007

398. Lemieux C, Gardam M, Evans G et al (2017) Longitudinal multicenter analysis of outcomes after cessation of control measures for vancomycinresistant enterococci. Infect Control Hosp Epidemiol 38(1):24-30

399. Muto CA, Jernigan JA, Ostrowsky BE et al (2003) SHEA guideline for preventing nosocomial transmission of multidrug-resistant strains of Staphylococcus aureus and enterococcus. Infect Control Hosp Epidemiol 24(5):362-386

400. Siegel JD, Rhinehart E, Jackson M, Chiarello L (2007) Management of multidrug-resistant organisms in health care settings, 2006. Am J

Infect Control 35(10 Suppl 2):S165-S193

401. Government of South Australia (SA Health)

(2017) Management of Patients with

Vancomycin-resistant Enterococci (VRE) Clinical

Guideline. Version 6.2. http://www.sahealth.

sa.gov.au/wps/wcm/connect/public+content/ sa+health+internet/resources/policies/vancomycin-resistant+enterococci+vre+patient+man agement+clinical+guideline. Zugegriffen: 22. März 2018

402. Lepelletier $D$, Berthelot $P$, Lucet JC, Fournier $S$, Jarlier V, Grandbastien B (2015) French recom mendations for the prevention of 'emerging extensively drug-resistant bacteria' (eXDR) crosstransmission. J Hosp Infect 90(3):186-195

403. Kluytmans-VandenBergh MFQ, Kluytmans JAJW, Voss A (2005) Dutch guideline for preventing nosocomial transmission of highly resistant microorganisms (HRMO). Infection 33(5-6):309313

404. Cookson BD, Macrae MB, Barrett SP et al (2006) Guidelines for the control of glycopeptideresistant enterococci in hospitals. J Hosp Infect $62(1): 6-21$ 